\title{
Chiral operators in two-dimensional $(0,2)$ theories and a test of triality
}

\author{
Jirui Guo, ${ }^{a}$ Bei $\mathrm{Jia}^{b}$ and Eric Sharpe ${ }^{a}$ \\ ${ }^{a}$ Department of Physics MC 0435, 850 West Campus Drive, \\ Virginia Tech, Blacksburg, VA 24061, U.S.A. \\ ${ }^{b}$ Theory Group, Physics Department, \\ University of Texas, Austin, TX 78712, U.S.A. \\ E-mail: jrkwok@vt.edu, beijia@physics.utexas.edu, ersharpe@vt.edu
}

ABSTRACT: In this paper we compute spaces of chiral operators in general two-dimensional $(0,2)$ nonlinear sigma models, both in theories twistable to the $\mathrm{A} / 2$ or $\mathrm{B} / 2$ model, as well as in non-twistable theories, and apply them to check recent duality conjectures. The fact that in a nonlinear sigma model, the Fock vacuum can act as a section of a line bundle on the target space plays a crucial role in our $(0,2)$ computations, so we begin with a review of this property. We also take this opportunity to show how even in $(2,2)$ theories, the Fock vacuum encodes in this way choices of target space spin structures, and discuss how such choices enter the A and B model topological field theories. We then compute chiral operators in general $(0,2)$ nonlinear sigma models, and apply them to the recent Gadde-Gukov-Putrov triality proposal, which says that certain triples of $(0,2)$ GLSMs should RG flow to nontrivial IR fixed points. We find that different UV theories in the same proposed universality class do not necessarily have the same space of chiral operators - but, the mismatched operators do not contribute to elliptic genera and are in nonintegrable representations of the proposed IR affine symmetry groups, suggesting that the mismatched states become massive along RG flow. We find this state matching in examples not only of different geometric phases of the same GLSMs, but also in phases of different GLSMs, indirectly verifying the triality proposal, and giving a clean demonstration that $(0,2)$ chiral rings are not topologically protected. We also check proposals for enhanced IR affine $E_{6}$ symmetries in one such model, verifying that (matching) chiral states in phases of corresponding GLSMs transform as 27's, $\overline{\mathbf{2 7}}$ 's.

Keywords: Supersymmetric gauge theory, Supersymmetry and Duality, Duality in Gauge Field Theories, Sigma Models

ARXIV EPRINT: 1501.00987 


\section{Contents}

1 Introduction 1

2 Chiral rings in two-dimensional $(0,2)$ theories 3

2.1 Review of chiral rings in $(2,2)$ theories and Fock vacuum subtleties 4

2.1.1 Fock vacua coupling to nontrivial bundles on the target 5

2.1.2 A, B model topological field theories and non-simply-connected targets 6

$\begin{array}{lll}2.2 & (0,2) \text { chiral states and rings } & 7\end{array}$

3 Application to triality 11

$\begin{array}{ll}3.1 & \text { Overview of triality } \\ 3.2 & 11\end{array}$

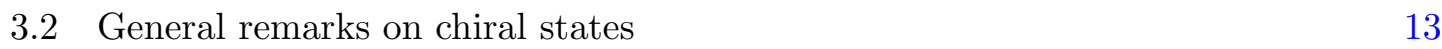

$\begin{array}{lll}3.2 .1 & \text { UV physics } & 13\end{array}$

$\begin{array}{lll}3.2 .2 & \text { IR physics } & 14\end{array}$

$\begin{array}{lll}3.3 & \text { Pseudo-topological twists } & 15\end{array}$

$\begin{array}{lll}3.4 & \text { First example } & 17\end{array}$

$\begin{array}{lll}\text { 3.4.1 First GLSM } & 17\end{array}$

3.4.2 Other GLSMs 23

3.5 Second example 26

3.5.1 First GLSM 26

3.5.2 Other GLSMs 30

3.6 Third example: $T_{222} \quad 31$

3.6.1 First GLSM 31

$\begin{array}{lll}3.6 .2 & \text { Other GLSMs } & 36\end{array}$

4 Conclusions $\quad 37$

$\begin{array}{ll}\text { A Bott-Borel-Weil } & 39\end{array}$

\section{Introduction}

Over the last few years we have seen a tremendous surge of development in two-dimensional GLSMs and theories with $(2,2)$ and $(0,2)$ supersymmetry. To give just a few examples, we now have examples of nonperturbatively-realized geometries [1-7], perturbative GLSMs for Pfaffians [2, 8, 9], non-birational GLSM phases [1, 3, 4], examples of closed strings on noncommutative resolutions $[4,7,10,11]$, localization techniques and new computations of, for example, Gromov-Witten invariants and elliptic genera (see for example [12-17]), and progress in heterotic string compactifications, ranging from nonperturbative correlations to new two-dimensional dualities and an understanding of non-Kähler moduli (see for example $[9,18-27])$. 
This paper concerns chiral states and rings in $(0,2)$ theories. Chiral rings have an extensive history in two-dimensional $(2,2)$ supersymmetric theories, providing tools such as quantum cohomology to help analyze quantum field theories. They also can exist in $(0,2)$ supersymmetric theories (see for example [26-36]), and are a current research topic.

As one particularly illuminating example, we apply chiral states to discuss triality proposals for two-dimensional $(0,2)$ theories, described in [24, 25]. Specifically, those papers proposed that all points in phase diagrams of certain triples of $(0,2)$ GLSMs should RG flow to the same IR fixed point, yielding a 'triality' relating three naively different twodimensional gauge theories. Now, many two-dimensional gauge theory dualities have a simple understanding as different presentations of the same IR geometry, as discussed in [9], but as also observed there, triality is different — of the six total geometric phases, there are three pairs such that each pair is associated with the same geometry, but the geometries associated to different pairs are simply different. Thus, it would be useful to have further insight into triality, as it cannot be understood as simply as other two-dimensional gauge dualities.

We begin in section 2 with a general overview of chiral states and rings in twodimensional $(0,2)$ theories. These have been described previously only in the $\mathrm{A} / 2$ and $\mathrm{B} / 2$ models; here, we describe chiral operators in general $(0,2)$ nonlinear sigma models. The correct counting of chiral states in two-dimensional $(0,2)$ nonlinear sigma models utilizes the fact that the Fock vacuum transforms as a section of a line bundle over the target space, a phenomenon closely related to the fractional fermion number property. This has been observed in a few other papers, but the basic idea still seems to be somewhat obscure to the community, so we take this opportunity to review the details. We also observe that even in $(2,2)$ theories, the Fock vacuum can be a section of a nontrivial line bundle, encoding choices of spin structure on the target space. When applied to $(0,2)$ theories, the resulting spectra are shown to satisfy basic consistency properties, such as invariance under Serre duality and also invariance under dualizing bundle factors.

In section 3 we apply chiral operators to study examples of triality. We begin with a brief overview of triality, and how in general terms one can keep track of all of the global symmetries in chiral operator computations (as some of the symmetries are realized in a nonobvious fashion, encoded implicitly in the Bott-Borel-Weil theorem), and also how in principle states in UV nonlinear sigma models, computed in terms of sheaf cohomology, should be related to state countings in the IR limit, which should be at least partially encoded in Lie algebra cohomology. We also briefly discuss pseudo-topological twists of examples of triality, to the $\mathrm{A} / 2$ and $\mathrm{B} / 2$ models.

Next, in each of three examples of triality, we compute chiral operators in two geometric phases in each of two GLSMs related by triality, keeping track of global symmetries, both to better understand triality and also to gain insight into properties of $(0,2)$ chiral states. We find that only a subset of all chiral states computed match between phases and GLSMs. However, $(0,2)$ chiral states, unlike $(2,2)$ chiral states, are not protected against nonperturbative corrections, and as RG flow will take the weakly-coupled nonlinear sigma models to a strong coupling regime, such a state mismatch should not be unexpected. As noted in $[24,25]$, the global symmetry groups should be enhanced to affine groups of certain 
levels in the IR, and chiral states should lie in integrable representations of those affine symmetry algebras. We check that the subset of the states that match between phases and GLSMs, all lie in integrable representations of the affine symmetry algebras predicted by $[24,25]$, consistent with the predictions of triality. The mismatched states all lie in non-integrable representations, which one would hope would not survive to the IR. Furthermore, the mismatched states all come in pairs whose contributions to at least leading terms in elliptic genera, refined by any of the computed global symmetries, cancel out, consistent with the conjecture that the mismatched states become massive along the RG flow. (In principle, pairs of massive states could also become massless along RG flow, but we did not observe any examples of such in the examples computed.) Thus, by counting chiral states in examples, we find nontrivial evidence for triality, as well as extremely clean examples of states varying along RG flow.

Such behavior along RG flow is of course typical of non-protected operators, but many workers in the $(0,2)$ community have sometimes implicitly assumed that $(0,2)$ chiral rings would be protected, hence triality provides clean examples stress-testing assumptions about $(0,2)$ chiral rings. (See also [37] which reached a related conclusion in certain other special cases.)

Furthermore, in some examples of triality the global symmetry algebras are further enhanced, and we find that the matching chiral states can be recombined into integrable representations of the larger symmetry algebras, again consistent with the predictions of $[24,25]$.

Finally, as the Bott-Borel-Weil theorem in mathematics plays a crucial role in our computations, in appendix A we include a short overview, so as to make this paper self-contained.

\section{Chiral rings in two-dimensional $(0,2)$ theories}

Part of the purpose of this paper is to compute chiral operators in general $(0,2)$ nonlinear sigma models, as a means of constructing tests of the Gadde-Gukov-Putrov triality proposal [24, 25].

To that end, let us first address chiral rings in gauged linear sigma models. These are two-dimensional gauge theories, so one might expect that chiral rings should be given as rings of gauge-invariant operators modulo relations determined by the superpotential, just as they are computed in four-dimensional gauge theories. Unfortunately, even in $(2,2)$ theories in two dimensions, this gives an incomplete result.

Consider, for example, the $(2,2)$ GLSM describing the quintic hypersurface in $\mathbb{P}^{4}$, a $\mathrm{U}(1)$ gauge theory with five fields $\phi_{i}$ of charge +1 and one field $p$ of charge -5 . The chiral ring computed as above includes operators of the form

$$
p\left(\text { degree } 5 \text { polynomial in } \phi_{i}\right)
$$

modulo relations of the form $p d G$, where $G$ is the quintic hypersurface. Certainly these form part of the chiral ring - in particular, these encode complex structure deformations in $H^{2,1}$. However, it is well-known that not all complex structure deformations of a hypersurface 
or complete intersection can be expressed as polynomials of the form above. Such nonalgebraic complex structure deformations contribute to cohomology $H^{2,1}$ and to the chiral ring in the nonlinear sigma model. Since the $(2,2)$ chiral ring lies in a topological subsector, in principle those same non-algebraic deformations ought to appear in the complete chiral ring of the GLSM. Unfortunately, it is not known at present how to present those elements of the chiral ring of the GLSM, those non-algebraic complex structure deformations, in terms of gauge-invariant operators.

Although we do not know how to build the complete chiral ring in a $(2,2)$ GLSM, we do know how to build the complete chiral ring in a $(2,2)$ nonlinear sigma model. Thus, in this paper we shall focus on chiral rings in nonlinear sigma models.

\subsection{Review of chiral rings in $(2,2)$ theories and Fock vacuum subtleties}

Let us begin our discussion of $(0,2)$ chiral rings with a brief review of pertinent aspects of chiral rings in $(2,2)$ supersymmetric nonlinear sigma models, focusing on some subtleties in Fock vacua that do not seem to be widely appreciated but which will play a crucial role in the $(0,2)$ generalization.

Consider a $(2,2)$ nonlinear sigma model on a complex Kähler manifold $X$. Let $\phi: \Sigma \rightarrow$ $X$ denote the worldsheet scalars, and $\psi_{ \pm}^{i, \bar{\imath}}$ worldsheet fermions coupling to the tangent bundle of $X$, in the standard fashion. Then, the chiral ring we will generalize to $(0,2)$ is the ring of states in $(\mathrm{R}, \mathrm{R})$ sectors, ${ }^{1}$ which following standard methods (see e.g. [38]) are of the form

$$
b_{\bar{\imath}_{1}, \cdots, \bar{z}_{n}, j_{1}, \cdots, j_{m}} \psi_{+}^{\bar{\tau}_{1}} \cdots \psi_{+}^{\bar{\tau}_{n}} \psi_{-}^{j_{1}} \cdots \psi_{-}^{j_{m}}|0\rangle
$$

The factor

$$
b_{\bar{\imath}_{1}, \cdots, \bar{\imath}_{n}, j_{1}, \cdots, j_{m}} \psi_{+}^{\bar{\tau}_{1}} \cdots \psi_{+}^{\bar{\imath}_{n}} \psi_{-}^{j_{1}} \cdots \psi_{-}^{j_{m}}
$$

has a standard understanding in terms of the cohomology of the space. What we will need in our discussion of $(0,2)$ chiral rings, and may be less widely understood, is that the Fock vacuum $|0\rangle$ might also couple to a nontrivial bundle on the target space $^{2}$ and contribute to the state counting. This phenomenon has also been discussed in [7, 40, 41], but as this phenomenon may be obscure, to make this paper self-contained, we will briefly review it here. We will also take this opportunity to describe how the same phenomenon arises even in $(2,2)$ theories, in describing spin structures on target spaces, something which to our

\footnotetext{
${ }^{1}$ Usually we compute states in R sectors rather than NS because the low-energy states can be obtained via a cohomology computation. For example, from [38, section 2], in an $N=2$ SCFT, in the R sector

$$
L_{0}=\left\{G_{0}^{-}, G_{0}^{+}\right\}+c / 12,
$$

and so $L_{0}-c / 12$ lives in cohomology, whereas in the NS sector,

$$
L_{0}=(1 / 4)\left\{G_{-1 / 2}^{-}, G_{+1 / 2}^{+}\right\}+(1 / 4)\left\{G_{+1 / 2}^{-}, G_{-1 / 2}^{-}\right\},
$$

which is not a cohomology computation, but rather an analogue of a harmonic representative computation. The two are related by supersymmetry and should give equivalent results, but the cohomology computation is significantly simpler, especially in the absence of an explicit target space metric.

${ }^{2}$ We should distinguish this from Fock vacua coupling to nontrivial bundles over a moduli space of SCFT's, say, which is well-known, see for example [39].
} 
knowledge has not previously appeared in the literature. (See also [42, 43] for a general discussion of spinors in strings, and [44, section 2] for a discussion of the same phenomenon in one-dimensional theories.)

\subsubsection{Fock vacua coupling to nontrivial bundles on the target}

We can understand this phenomenon as follows. In a chiral $\mathrm{R}$ sector, the Fock vacuum couples to $\left(K_{X}\right)^{ \pm 1 / 2}$ in general. This follows from the usual multiplicity of Fock vacua in the presence of periodic fermions. Schematically, if we define two vacua $|0\rangle,|0\rangle^{\prime}$ by

$$
\psi_{0}^{i}|0\rangle^{\prime}=0, \quad \psi_{0}^{\bar{\imath}}|0\rangle=0,
$$

then

$$
|0\rangle^{\prime}=\left(\prod_{i} \psi_{0}^{i}\right)|0\rangle, \quad|0\rangle=\left(\prod_{\bar{\imath}} \psi_{0}^{\bar{\imath}}\right)|0\rangle^{\prime} .
$$

Since

$$
\prod_{i} \psi_{0}^{i} \sim K_{X}^{-1}, \prod_{\bar{\imath}} \psi_{0}^{\bar{\imath}} \sim K_{X}
$$

then, just as in fractional charges, $|0\rangle^{\prime}$ transforms as a section of $\left(K_{X}\right)^{-1 / 2}$ and $|0\rangle$ transforms as a section of $\left(K_{X}\right)^{+1 / 2}$.

In the case of a $(2,2)$ theory, the Fock vacuum couples to

$$
\left(K_{X}\right)^{+1 / 2} \otimes\left(K_{X}\right)^{-1 / 2}
$$

one factor for left-movers, the other for right-movers.

If $X$ is simply-connected, the square roots $\left(K_{X}\right)^{ \pm 1 / 2}$, if they exist, are uniquely determined, and the Fock vacuum couples to a trivial bundle. (If they do not exist in a given sector, then that sector is physically inconsistent.)

More generally, if $X$ is not simply-connected, then there will be multiple different square roots $\left(K_{X}\right)^{ \pm 1}$. These different choices of square roots correspond to different choices of spin structure on the target space $X$, as spinors on a complex Kähler manifold can be expressed in the form [45, section II.3]

$$
\wedge^{\bullet} T X \otimes\left(K_{X}\right)^{+1 / 2} .
$$

If $X=T^{2}$, for example, the different square roots $\left(K_{X}\right)^{1 / 2}$ simply correspond to choices of periodic and antiperiodic boundary conditions around the legs of the torus.

To compute the chiral ring, we must specify two square roots, one for left-movers, another for right-movers. Which square roots should appear, associated to left- and rightmovers, is part of the specification of the nonlinear sigma model. In other words, just as one must specify a metric and $B$ field on $X$ in order to define a nonlinear sigma model, if $X$ is not simply-connected then in addition one must also specify a spin structure on $X$, and that choice of spin structure enters worldsheet physics via Fock vacua, as above.

In this paper, we will work with simply-connected spaces. However, in the $(0,2)$ case, Fock vacua will couple to bundles of the form $(\operatorname{det} \mathcal{E})^{-1 / 2} \otimes K_{X}^{+1 / 2}$, and so even if there is 
no ambiguity, the bundle can be nontrivial. More generally, suppose $|0\rangle \in \Gamma(L)$ for some line bundle $L$. If $L$ has no sections at all, this merely implies that the Fock vacuum is not BRST-closed: we still have a Fock vacuum, it merely does not define a state by itself, analogous to tachyonic states projected out of closed bosonic string spectra. At a different extreme, if $L$ admits multiple sections, this is merely another source of multiplicity beyond that provided by Fermi zero modes.

For completeness, and since this fact does not yet seem to be widely appreciated in the literature, let us explore some of the implications of the statement above on the $(2,2)$ locus.

If $X$ is Calabi-Yau, so that $K_{X}$ is trivial, then there is a canonical ${ }^{3}$ trivial square root, specifically $\left(K_{X}\right)^{+1 / 2}=\mathcal{O}_{X}$. (If $K_{X}$ is nontrivial, then in general there will not be any canonical choice of square root, if square roots in fact exist.) Only in that canonical trivial spin structure on a Calabi-Yau does there exist a nowhere-zero covariantly constant spinor [46]. (For example, on $T^{2}$, only in the (R,R) spin structure does $K^{1 / 2}$ have a section.)

Since the anomaly in the left and right $\mathrm{U}(1)_{R}$ symmetries is determined by $K_{X}$ and not the spin structure, if $X$ is Calabi-Yau this theory should flow to a nontrivial $(2,2)$ SCFT, even if one chooses nontrivial left- or right-spin structures. However, the target space string theories are not likely well-defined if either spin structure is nontrivial [42, 43, 47]. Even if the target space string theory is well-defined, spacetime supersymmetry must surely be broken if either spin structure is nontrivial. In an SCFT associated to a Calabi-Yau compactification, there is an isomorphism between R and NS sector states: spectral flow rotates one into the other. If $\left(K_{X}\right)^{1 / 2}$ is nontrivial, however, that isomorphism is broken. After all, the Fock vacuum ambiguity which leads to this interpretation in terms of $\left(K_{X}\right)^{1 / 2}$ only exists in the R sector, not the NS sector, and so the states that arise in the R sector are necessarily distinct. ${ }^{4}$ Put another way, spectral flow relates NS sector states to the $\mathrm{R}$ sector states associated with trivial spin structures, instead of the given spin structures. In effect, this is a further condition for spacetime supersymmetry in $N=2$ SCFT's, beyond the familiar statement that the difference of left and right charges should be integral [38, eq. (2.3)].

In orbifolds, an example of the effect of having $\left(K_{X}\right)^{1 / 2}$ nontrivial while $K_{X}$ is trivial is given by the Scherk-Schwarz mechanism [48], which breaks supersymmetry in orbifolds by assigning different boundary conditions to fermions than to bosons. (In full string theories, such boundary conditions might also contribute to e.g. failures of level-matching or modular invariance, but to decide the matter, one would need to specify the rest of the CFT needed for a critical string.)

\subsubsection{A, B model topological field theories and non-simply-connected targets}

For completeness, let us also briefly discuss the A and B model topological field theories for non-simply-connected target spaces. In both cases, the Fock vacuum couples to the

\footnotetext{
${ }^{3}$ More generally, the $n$th roots of the structure sheaf form a finite group which is canonically $\operatorname{Hom}\left(\pi_{1}(X), \mu_{n}\right)$ where $\mu_{n}$ is the group of $n$th roots of unity [46].

${ }^{4}$ The spectral flow operator that takes NS to the relevant $\mathrm{R}$ and conversely has $\theta=1 / 2$ in the notation of [38], and charge $\pm c / 6$, and formally would be associated to a square root of the canonical bundle. That spectral flow operator would be different from the one in [38], which maps NS to the R sector for the canonical spin structure. The one relevant here could not be expressed merely as the exponential of a boson, unlike the one in [38].
} 
ratio of square roots in equation (2.1). In conventional treatments of the $\mathrm{A}$ and $\mathrm{B}$ models such as [49], the two square roots are assumed identical, so that the bundle is trivial. (If $X$ does not admit a spin structure, then $\left(K_{X}\right)^{1 / 2}$ does not exist as an honest bundle, only a 'twisted' bundle; however, by formally identifying the contributions from left- and right-moving sectors, we can still make sense of the RR sector, and hence the topological field theories.)

More generally, if the square roots are not identical, then the Fock vacuum couples to a nontrivial bundle. The two topological field theories appear to still be well-defined, but their interpretations are slightly different. The operators in the A model continue to be counted by

$$
H^{q}\left(\Omega_{X}^{p}\right)
$$

but the states are now counted by

$$
H^{q}\left(\Omega_{X}^{p} \otimes\left(K_{X}\right)^{+1 / 2} \otimes\left(K_{X}\right)^{-1 / 2}\right) .
$$

Similarly, the operators in the B model continue to be counted by

$$
H^{q}\left(\wedge^{p} T X\right)
$$

but the states are now counted by

$$
H^{q}\left(\wedge^{p} T X \otimes\left(K_{X}\right)^{+1 / 2} \otimes\left(K_{X}\right)^{-1 / 2}\right) .
$$

We do not interpret this as a violation of the state-operator corresopndence, which refers to the SL $(2, \mathbb{C})$-invariant NS-NS vacuum, but instead in terms of spectral flow. For example, when A model three-point correlation functions are interpreted in a physical theory, the physical correlation function takes the form

$$
\text { (spacetime spinor) (spacetime boson) (spacetime spinor), }
$$

where the spinor structure is encoded in the Fock vacuum. We interpret the issue above similarly.

That said, correlation functions of local observables are unchanged by the choices of target-space spin structure, as the combination of $|0\rangle$ and $\langle 0|$ result in a factor of

$$
\left(\left(K_{X}\right)^{+1 / 2} \otimes\left(K_{X}\right)^{-1 / 2}\right)^{2} \cong K_{X} \otimes K_{X}^{-1} \cong \mathcal{O}_{X}
$$

It is possible that nonlocal ${ }^{5}$ observables may be able to detect the spin structure.

\section{$2.2(0,2)$ chiral states and rings}

Briefly, the $(0,2)$ chiral states in which we are interested are the "massless" or zero-energy elements of the ring of states annihilated by a right supercharge. The set of all states annihilated by a right supercharge, an infinite tower, forms a ring. In this paper, we will focus on the "massless" elements of that ring, which form a finite-dimensional subset.

\footnotetext{
${ }^{5}$ We would like to thank H. Jockers for observing this possibility.
} 
The elements of the right-chiral ring with fixed conformal dimension need not form a ring. Surprisingly, however, under certain circumstances [36] it can be shown that the OPE's nevertheless close into themselves. Specifically, if the bundle rank is less than eight, then the massless chiral states in the $\mathrm{A} / 2$ model will close into a ring, at least in patches on the moduli space.

Let us make explicit what we mean by the $(0,2)$ chiral states in a $(0,2)$ nonlinear sigma model on a space $X$ with holomorphic vector bundle $\mathcal{E}$, satisfying the conditions ${ }^{6}$

$$
\operatorname{ch}_{2}(\mathcal{E})=\operatorname{ch}_{2}(T X), \quad c_{1}(\mathcal{E}) \equiv c_{1}(T X) \bmod 2 .
$$

In the $(\mathrm{R}, \mathrm{R})$ sector, slightly generalizing the old analysis of [50], the states in the worldsheet theory are of the form

$$
b_{\bar{\imath}_{1}, \cdots, \bar{z}_{n}, a_{1}, \cdots, a_{m}} \lambda_{-}^{a_{1}} \cdots \lambda_{-}^{a_{m}} \psi_{+}^{\bar{\tau}_{1}} \cdots \psi_{+}^{\bar{z}_{n}}|0\rangle
$$

for the Fock vacuum defined by

$$
\psi_{+}^{i}|0\rangle=0=\lambda_{-}^{\bar{a}}|0\rangle .
$$

The Fock vacuum defined as above transforms as a section of the bundle

$$
(\operatorname{det} \mathcal{E})^{-1 / 2} \otimes K_{X}^{+1 / 2}
$$

essentially as a consequence of its fractional charges under global symmetries, as discussed in section 2.1. Following standard methods (for example [50]), since the right supercharge can be identified with $\bar{\partial}$, the states above realize a Dolbeault representation of the sheaf cohomology groups

$$
H^{n}\left(X,\left(\wedge^{m} \mathcal{E}\right) \otimes(\operatorname{det} \mathcal{E})^{-1 / 2} \otimes K_{X}^{+1 / 2}\right) .
$$

(This is a special case of the general result for massless spectra of heterotic strings on stacks described in [40, appendix A].)

The ratio of square roots will exist whenever ${ }^{7}$

$$
c_{1}(\mathcal{E}) \equiv c_{1}(T X) \bmod 2,
$$

\footnotetext{
${ }^{6}$ The second condition suffices to define the theory in an $(\mathrm{R}, \mathrm{R})$ sector. In more general sectors, one would need to separately require that $c_{1}(\mathcal{E})$ and $c_{1}(T X)$ vanish $\bmod 2$; however, in this paper we will only be concerned with the RR sector.

${ }^{7}$ In GLSMs, the analogous constraint for a single U(1) would be the statement

$$
\sum_{\alpha} q_{L, \alpha} \equiv \sum_{\beta} q_{R, \beta} \bmod 2,
$$

relating the sum of charges of left- and right-moving fields. However, note that since

$$
\sum_{\alpha} q_{\alpha}^{2} \equiv \sum_{\alpha} q_{\alpha} \bmod 2
$$

the anomaly cancellation condition

$$
\sum_{\alpha} q_{L, \alpha}^{2}=\sum_{\beta} q_{R, \beta}^{2}
$$

implies the statement above. See also [40, appendix A.4] for a discussion of this condition as it appears in orbifolds and related theories.
} 
which is typically taken as a consistency condition on heterotic nonlinear sigma models. (In fact, to make sense of the (R,NS) and (NS,R) sectors, we must require that $\operatorname{det} \mathcal{E}$ and $K_{X}$ separately admit square roots, which requires $c_{1}(E) \equiv 0 \bmod 2$ and separately $c_{1}(T X) \equiv 0$ mod 2. For our purposes in this paper, we will focus on $(R, R)$ sectors, and so the condition above suffices.) Thus, square roots will exist in cases of interest.

As an aside, in a typical perturbative heterotic compactification, it is taken that both $K_{X}$ and $\operatorname{det} \mathcal{E}$ are trivial. In this case, each has a canonical trivial square root.

Now, beyond the ambiguities just described, there are different choices one could make for R sector Fock vacua. For example, we could instead consider the Fock vacuum defined by

$$
\psi_{+}^{i}|0\rangle=0=\lambda_{-}^{a}|0\rangle,
$$

which instead couples to

$$
(\operatorname{det} \mathcal{E})^{+1 / 2} \otimes K_{X}^{+1 / 2}
$$

In this case, states would be enumerated in the form

$$
b_{\bar{\imath}_{1}, \cdots, \bar{\imath}_{n}, \bar{a}_{1}, \cdots, \bar{a}_{m}} \lambda_{-}^{\bar{a}_{1}} \cdots \lambda_{-}^{\bar{a}_{m}} \psi_{+}^{\bar{\tau}_{1}} \cdots \psi_{+}^{\bar{z}_{n}}|0\rangle,
$$

and counted by

$$
H^{n}\left(X,\left(\wedge^{m} \mathcal{E}^{*}\right) \otimes(\operatorname{det} \mathcal{E})^{+1 / 2} \otimes K_{X}^{+1 / 2}\right)
$$

However, the choice of Fock vacuum should not change the states, and that is reflected in mathematical dualities. For example, using the fact that

$$
\wedge^{m} \mathcal{E}^{*}=\left(\wedge^{r-m} \mathcal{E}\right) \otimes\left(\operatorname{det} \mathcal{E}^{*}\right)
$$

(for $r$ the rank of $\mathcal{E}$ ), it is easy to check that

$$
H^{n}\left(X,\left(\wedge^{m} \mathcal{E}^{*}\right) \otimes(\operatorname{det} \mathcal{E})^{+1 / 2} \otimes K_{X}^{+1 / 2}\right)=H^{n}\left(X,\left(\wedge^{r-m} \mathcal{E}\right) \otimes(\operatorname{det} \mathcal{E})^{-1 / 2} \otimes K_{X}^{+1 / 2}\right),
$$

and so we see that these are merely two different descriptions of the same set of states:

$$
H^{\bullet}\left(X,\left(\wedge^{\bullet} \mathcal{E}\right) \otimes(\operatorname{det} \mathcal{E})^{-1 / 2} \otimes K_{X}^{+1 / 2}\right)=H^{\bullet}\left(X,\left(\wedge^{\bullet} \mathcal{E}^{*}\right) \otimes(\operatorname{det} \mathcal{E})^{+1 / 2} \otimes K_{X}^{+1 / 2}\right) .
$$

Thus, the choice of conventions in picking Fock vacua do not alter the set of states.

Let us list a few consistency checks:

- In the $\mathrm{A} / 2$ model, where $\operatorname{det} \mathcal{E}^{*} \cong K_{X}$, the states should be counted by $H^{\bullet}\left(X, \wedge^{\bullet} \mathcal{E}^{*}\right)$, and in the $\mathrm{B} / 2$ model, where $\operatorname{det} \mathcal{E} \cong K_{X}$, the states should be counted by $H^{\bullet}\left(X, \wedge^{\bullet} \mathcal{E}\right)$.

- On the $(2,2)$ locus, the states should be counted by

$$
H^{\bullet}\left(X, \wedge^{\bullet} T^{*} X\right)=H^{\bullet}\left(X, \wedge^{\bullet} T X \otimes K_{X}\right)
$$

- The states should be counted by sheaf cohomology groups that close into themselves under Serre duality. 
- The structure above is compatible with the left Ramond elliptic genus of $(0,2)$ nonlinear sigma models. As discussed in e.g. [9, 51, 52], the leading term in the elliptic genus of a sigma model on $X$ with bundle $\mathcal{E}$ is proportional to

$\int_{X} \hat{A}(T X) \wedge \operatorname{ch}\left((\operatorname{det} \mathcal{E})^{-1 / 2} \wedge_{-1} \mathcal{E}\right)=\int_{X} \operatorname{td}(T X) \wedge \operatorname{ch}\left(K_{X}^{+1 / 2} \otimes(\operatorname{det} \mathcal{E})^{-1 / 2} \wedge_{-1} \mathcal{E}\right)$,

which is the Hirzebruch-Riemann-Roch index appropriate for the sheaf cohomology groups above.

- The states should be invariant under $\mathcal{E} \mapsto \mathcal{E}^{*}$ (which should swap the A/2, B/2 models) (see e.g. [29]).

- If the bundle $\mathcal{E}$ is reducible, the states should be invariant under separately dualizing factors. We can check this explicitly as follows. Suppose $\mathcal{E}$ splits holomorphically, $\mathcal{E}=\mathcal{A} \oplus \mathcal{B}$, of ranks $r_{1}, r_{2}$, respectively. Using

$$
\wedge^{\bullet} \mathcal{E}=\sum_{i+j=\bullet} \wedge^{i} \mathcal{A} \otimes \wedge^{j} \mathcal{B}
$$

we have

$$
\begin{aligned}
H^{\bullet} & \left(X,\left(\wedge^{i} \mathcal{A}\right) \otimes\left(\wedge^{j} \mathcal{B}\right) \otimes(\operatorname{det} \mathcal{A})^{-1 / 2} \otimes(\operatorname{det} \mathcal{B})^{-1 / 2} \otimes K_{X}^{+1 / 2}\right) \\
& =H^{\bullet}\left(X,\left(\wedge^{i} \mathcal{A}\right) \otimes\left(\wedge^{r_{2}-j} \mathcal{B}^{*}\right) \otimes(\operatorname{det} \mathcal{B}) \otimes(\operatorname{det} \mathcal{A})^{-1 / 2} \otimes(\operatorname{det} \mathcal{B})^{-1 / 2} \otimes K_{X}^{+1 / 2}\right) \\
& =H^{\bullet}\left(X,\left(\wedge^{i} \mathcal{A}\right) \otimes\left(\wedge^{r_{2}-j} \mathcal{B}^{*}\right) \otimes(\operatorname{det} \mathcal{A})^{-1 / 2} \otimes\left(\operatorname{det} \mathcal{B}^{*}\right)^{-1 / 2} \otimes K_{X}^{+1 / 2}\right)
\end{aligned}
$$

Thus, the spectrum remains invariant if we replace $\mathcal{A} \oplus \mathcal{B}$ by $\mathcal{A} \oplus \mathcal{B}^{*}$. At some level, this reflects the fact that shuffling between Fock vacua does not change the set of states, and so is a self-consistency test.

The sheaf cohomology groups above in (2.2) can straightforwardly be shown to satisfy all of the conditions above.

So far we have only discussed the additive structure of the chiral states; however, in special cases, there are also results on product structures. For example, in the special cases $\operatorname{det} \mathcal{E} \cong K_{X}^{*}$, there exists a pseudo-topological twists known as the $\mathrm{A} / 2$ model, for which nonperturbative corrections to product structures have been computed for $X$ a toric variety and $\mathcal{E}$ a deformation of the tangent bundle, see for example [26-31, 36]. In this paper we will focus on additive structures only.

Finally, let us discuss the behavior of these states under deformations and RG flow. In a $(2,2)$ theory, the chiral states live in a topologically protected subsector, and so one expects to have the same additive structure in the chiral rings everywhere along RG flow and under deformations. For example, this is the physics reason why the Hodge numbers of CalabiYau's are the same in different geometric phases of the same GLSM. (In mathematics, this result is a consequence of motivic integration [53].)

By contrast, in $(0,2)$ theories, the chiral ring $Q$-cohomology computation is protected only against perturbative corrections, for the same reasons that the $(0,2)$ superpotential 
is not perturbatively renormalized. In applications such as [50], where RG flow stays in weakly-coupled regimes, $Q$-cohomology can be reliably used to count states. By contrast, in this paper we compute $Q$-cohomology in weakly-coupled UV nonlinear sigma models which RG flow to strong coupling. As a result, one should expect that our $Q$-cohomology computations above will not necessarily give the correct IR spectrum, but rather additional states could enter or leave along the RG flow, and in fact that is precisely what we find.

\section{Application to triality}

\subsection{Overview of triality}

It was proposed in [24] that triples of $(0,2)$ GLSMs might flow to the same IR fixed point. One starts with a $(0,2) \mathrm{U}(k)$ GLSM:

\begin{tabular}{|ccccc|}
\hline & type & multiplicity & $s u(k)$ & $u(1)$ \\
\hline$\Phi$ & chiral & $n$ & $\mathbf{k}$ & 1 \\
$P$ & chiral & $B$ & $\overline{\mathbf{k}}$ & -1 \\
$\Gamma$ & Fermi & $n B$ & $\mathbf{1}$ & 0 \\
$\Psi$ & Fermi & $A$ & $\overline{\mathbf{k}}$ & -1 \\
$\lambda$ & fermion & 1 & $a d$ & 0 \\
$\Omega$ & Fermi & 2 & $\mathbf{1}$ & $k$ \\
\hline
\end{tabular}

with a $(0,2)$ superpotential $W=\Gamma P \Phi$, where $B=2 k+A-n$. This GLSM was argued to be dual to a $(0,2) \mathrm{U}(n-k)$ GLSM:

\begin{tabular}{|ccccc|}
\hline & type & multiplicity & $s u(k)$ & $u(1)$ \\
\hline$\tilde{\Phi}$ & chiral & $n$ & $\mathbf{k}$ & 1 \\
$\tilde{P}$ & chiral & $A$ & $\overline{\mathbf{k}}$ & -1 \\
$\tilde{\Gamma}$ & Fermi & $n A$ & $\mathbf{1}$ & 0 \\
$\tilde{\Psi}$ & Fermi & $B$ & $\overline{\mathbf{k}}$ & -1 \\
$\tilde{\lambda}$ & fermion & 1 & $a d$ & 0 \\
$\tilde{\Omega}$ & Fermi & 2 & $\mathbf{1}$ & $k$ \\
\hline
\end{tabular}

with a $(0,2)$ superpotential $\tilde{W}=\tilde{\Gamma} \tilde{P} \tilde{\Phi}$. A further step of duality move leads to yet a third $(0,2)$ GLSM with a $\mathrm{U}(A-n+k)$ gauge group:

\begin{tabular}{|ccccc|}
\hline & type & multiplicity & $s u(k)$ & $u(1)$ \\
\hline$\Phi^{\prime}$ & chiral & $B$ & $\mathbf{k}$ & 1 \\
$P^{\prime}$ & chiral & $n$ & $\overline{\mathbf{k}}$ & -1 \\
$\Gamma^{\prime}$ & Fermi & $n B$ & $\mathbf{1}$ & 0 \\
$\Psi^{\prime}$ & Fermi & $A$ & $\overline{\mathbf{k}}$ & -1 \\
$\lambda^{\prime}$ & fermion & 1 & $a d$ & 0 \\
$\Omega^{\prime}$ & Fermi & 2 & $\mathbf{1}$ & $k$ \\
\hline
\end{tabular}

with a $(0,2)$ superpotential $W^{\prime}=\Gamma^{\prime} P^{\prime} \Phi^{\prime}$. 
This phenomenon, labelled "triality," can be understood as follows. If we integrate out the gauge field, then the large-radius limit of the first $(0,2)$ theory can be understood as a nonlinear sigma model on

$$
X_{1}=G(k, n)
$$

with bundle

$$
\mathcal{E}_{1}=S^{A} \oplus\left(Q^{*}\right)^{2 k+A-n} \oplus\left(\operatorname{det} S^{*}\right)^{2},
$$

where $S$ denotes the universal subbundle on $G(k, n)$, and $Q$ the universal quotient bundle on $G(k, n)$. This theory has four flavor symmetries, three of which rotate bundle factors

$$
\mathrm{SU}(A) \times \mathrm{SU}(2 k+A-n) \times \mathrm{SU}(2),
$$

and the fourth of which, $\mathrm{SU}(n)$, acts on the base. The other theories related by triality can be obtained by cyclically permuting

$$
A, \quad 2 k+A-n, \quad n
$$

and simultaneously replacing $k$ by $n-k$. For example, the large-radius limit of the second theory is given by a nonlinear sigma model on

$$
X_{2}=G(n-k, A)
$$

with bundle

$$
\mathcal{E}_{2}=S^{2 k+A-n} \oplus\left(Q^{*}\right)^{n} \oplus\left(\operatorname{det} S^{*}\right)^{2},
$$

and the large-radius limit of the third theory is given by a nonlinear sigma model on

$$
X_{3}=G(A-n+k, 2 k+A-n)
$$

with bundle

$$
\mathcal{E}_{3}=S^{n} \oplus\left(Q^{*}\right)^{A} \oplus\left(\operatorname{det} S^{*}\right)^{2} .
$$

Now, in order for the geometric description above to make sense, the values of $n, A$, and $k$ are constrained. For example, to make sense of $G(A-n+k, 2 k+A-n)$, we require

$$
0<A-n+k<2 k+A-n \text {. }
$$

In addition, in order for the triality to be interesting, we would also like supersymmetry to remain unbroken, which can be checked by e.g. computing elliptic genera as refined Witten indices. Happily, these two requirements - that the geometric description be sensible, and that supersymmetry be unbroken - coincide in these theories.

In the UV, in addition to some nonanomalous $\mathrm{U}(1)$ 's, the theories above have nonanomalous

$$
\mathrm{SU}(n) \times \mathrm{SU}(A) \times \mathrm{SU}(2 k+A-n) \times \mathrm{SU}(2)
$$

symmetries, as discussed above. It was proposed in [25] that in the IR, the theories above flow to a common nontrivial SCFT, in which the global flavor symmetries above are enhanced to affine symmetries [25, eq. (3.1)]

$$
\mathrm{SU}(n)_{k+A-n} \times \mathrm{SU}(A)_{k} \times \mathrm{SU}(2 k+A-n)_{n-k} \times \mathrm{SU}(2)_{1} .
$$


By examining chiral states among the UV theories above, we will give nontrivial evidence that the different UV theories flow to the same IR fixed point and have the IR affine symmetries indicated above.

\subsection{General remarks on chiral states}

\subsubsection{UV physics}

Let us focus on the first model in the last section, a heterotic nonlinear sigma model described by the space and bundle

$$
X=G(k, n), \quad \mathcal{E}=S^{A} \oplus\left(Q^{*}\right)^{2 k+A-n} \oplus\left(\operatorname{det} S^{*}\right)^{2} .
$$

To compute the chiral states, we first need to compute the bundle to which the Fock vacuum couples. To that end, in the model above,

$$
\operatorname{det} \mathcal{E}=\mathcal{O}(-(A-2)-(2 k+A-n))=\mathcal{O}(-2 k-2 A+n+2),
$$

and

$$
K_{X}=\mathcal{O}(-n)
$$

hence

$$
(\operatorname{det} \mathcal{E}) \otimes K_{X}=\mathcal{O}(-2 k-2 A+2), \quad(\operatorname{det} \mathcal{E})^{-1} \otimes K_{X}=\mathcal{O}(2 k+2 A-2 n-2),
$$

and so a square root always exists. Furthermore, since the Grassmannian is simplyconnected, that square root is unique, and defines the bundle to which the Fock vacuum couples.

Now, in this paper we are interested in more than merely counting states - we also want to keep track of global symmetry representations. To that end, it will be useful to describe $X$ and $\mathcal{E}$ in terms of vector spaces defining fundamental representations of global symmetry groups. For example, we will describe the model above as

$$
X=G\left(k, \tilde{V}^{*}\right), \quad \mathcal{E}=U \otimes S \oplus V \otimes Q^{*} \oplus W \otimes \operatorname{det} S^{*},
$$

where $U, V, W$, and $\tilde{V}$ are vector spaces of dimensions $A, 2 k+A-n, 2$, and $n$, respectively.

The original UV GLSM has a

$$
\mathrm{SU}(A) \times \mathrm{SU}(2 k+A-n) \times \mathrm{SU}(2) \times \mathrm{SU}(n)=\mathrm{SU}(U) \times \mathrm{SU}(V) \times \mathrm{SU}(W) \times \mathrm{SU}(\tilde{V})
$$

symmetry, but in the sheaf cohomology groups, naively only the

$$
\mathrm{SU}(A) \times \mathrm{SU}(2 k+A-n) \times \mathrm{SU}(2)=\mathrm{SU}(U) \times \mathrm{SU}(V) \times \mathrm{SU}(W)
$$

subgroup is explicit, in its action on the left-moving fermions. The remaining $\mathrm{SU}(n)=$ $\mathrm{SU}(\tilde{V})$, which acts on the base, is made manifest via the Bott-Borel-Weil theorem, reviewed in appendix A, which expresses sheaf cohomology of homogeneous vector bundles (including the $\mathcal{E}$ above) on Grassmannians $G(k, n)$ in terms of representations of $\mathrm{U}(n)$, making the relationship explicit. 
To that end, when computing chiral rings, it will be important to keep track of the difference between factors of e.g. $S$ and $Q^{*}$. As holomorphic bundles, for example $\operatorname{det} S \cong$ $\operatorname{det} Q^{*}$, but they define different representations of the parabolic subgroup $G L(k) \times G L(n-$ $k$ ) of $G L(n)$, and the difference will manifest via Bott-Borel-Weil in terms of the precise representation of $\mathrm{U}(n)$ appearing. For example, if $n=2$, then on $G\left(1, \tilde{V}^{*}\right)=\mathbb{P}^{1}, S^{*} \otimes Q^{*} \cong$ $\mathcal{O}$. However, applying Bott-Borel-Weil, we find

$$
\begin{aligned}
H^{\bullet}(\mathcal{O}) & =\mathbb{C} \delta^{\bullet, 0}, \\
H^{\bullet}\left(S^{*} \otimes Q^{*}\right) & =\wedge^{2} \tilde{V} \delta^{\bullet, 0} .
\end{aligned}
$$

The two sheaf cohomology groups have the same dimension - as they should, since the bundles are isomorphic as holomorphic bundles - but encode different representations of $G L(\tilde{V})$. Thus, for example, when specifying Fock vacuum bundles, we must specify not only a holomorphic line bundle, but in addition a precise representation of the parabolic subgroup, i.e. a precise description as powers of $S^{*}$ and $Q^{*}$.

\subsubsection{IR physics}

In principle, we would like to compare the UV sheaf cohomology groups to the corresponding $\mathrm{R}$ sector states in the IR theory, built from a right-moving part of a Kazama-Suzuki coset and a left-moving Kac-Moody algebra [25]. Now, for a $(2,2)$ Kazama-Suzuki coset $G / H$, it was argued in [38, section 5] that part of the chiral ring is given as the Lie algebra cohomology

$$
H^{\bullet}\left(\mathfrak{t}_{+}, V_{\lambda}\right)
$$

where $\mathfrak{g}=\mathfrak{h}+\mathfrak{t}_{+}+\mathfrak{t}_{-}$is a decomposition of the Lie algebra of $G, \mathfrak{h}$ the Lie algebra of $H$, and $V_{\lambda}$ is a $\mathfrak{g}$-module corresponding to the representation of $G$ corresponding to the ground state, i.e. an integrable representation. In the present case, for the IR limit in triality, one would similarly expect that some of the chiral states can be expressed in the form of Lie algebra cohomology

$$
H^{\bullet}\left(\mathfrak{t}_{+}, M\right),
$$

where $M$ is a $\mathfrak{p}$-module determined in part by the left-moving Kac-Moody algebra. Now, this description is incomplete - even for $(2,2)$ Kazama-Suzuki cosets, it is not believed that the Lie algebra cohomology provides a full description [38, 54], hence we cannot use this to try to completely enumerate IR states to compare to UV states.

That said, one could still ask how the sheaf cohomology groups appearing in the UV theory could be related, even in principle, to the Lie algebra cohomology groups that appear, at least incompletely, in the IR. The answer is provided by another version of the Bott-Borel-Weil theorem [55, 56]. As observed above, Bott-Borel-Weil naturally says that for a homogeneous bundle $\mathcal{E}_{\xi}$ on $G / P$ defined by a representation $\xi$ of $P$, the sheaf cohomology groups

$$
H^{\bullet}\left(G / P, \mathcal{E}_{\xi}\right)
$$

naturally come in representations of $G$. It was observed in $[55,56]$ that the Lie algebra cohomology groups

$$
H^{\bullet}\left(\mathfrak{t}_{+}, V_{\lambda}\right)
$$


(for $\mathfrak{t}_{+}$as above and $\lambda$ a representation of $G$ ) naturally come in representations of $P$. Moreover, these sheaf cohomology and Lie algebra cohomology groups are closely intertwined: the multiplicity of representation $\lambda$ of $G$ in $H^{j}\left(G / P, \mathcal{E}_{\xi}\right)$ matches the multiplicity of $\xi$ in $H^{j}\left(\mathfrak{t}_{+}, V_{\lambda}\right)$, or more simply

$$
H^{j}\left(G / P, \mathcal{E}_{\xi}\right)_{\lambda}=H^{j}\left(\mathfrak{t}_{+}, V_{\lambda}\right)_{\xi},
$$

where, for example,

$$
H^{j}\left(G / P, \mathcal{E}_{\xi}\right)=\sum_{\lambda} V_{\lambda} \otimes H^{j}\left(G / P, \mathcal{E}_{\xi}\right)_{\lambda} .
$$

In other words, if $\lambda$ is a five-dimensional representation, for example, and the sheaf cohomology group is $V_{\lambda}$, then

$$
H^{j}\left(G / P, \mathcal{E}_{\xi}\right)_{\mu}=\left\{\begin{array}{l}
\mathbb{C} \mu=\lambda, \\
0 \mu \neq \lambda,
\end{array}\right.
$$

so that one copy of the five-dimensional $V_{\lambda}$ appears, not five.

In these conventions,

$$
H^{\bullet}\left(G / P, \mathcal{E}_{\xi}\right)=\sum_{\mu} H^{\bullet}\left(G / P, \mathcal{E}_{\xi}\right)_{\mu} \otimes V_{\mu}
$$

and

$$
H^{\bullet}\left(\mathfrak{n}, V_{\lambda}\right)=\sum_{\zeta} H^{\bullet}\left(\mathfrak{n}, V_{\lambda}\right)_{\zeta} \otimes V_{\zeta}
$$

hence, for example,

$$
H^{\bullet}\left(G / P, \mathcal{E}_{\xi}\right)=\sum_{\mu} H^{\bullet}\left(\mathfrak{t}_{+}, V_{\mu}\right)_{\xi} \otimes V_{\mu}
$$

That said, in principle the $\mathfrak{t}_{+}$pertinent to the IR theory should be derived from a slightly more complicated Grassmannian, so what we have outlined is not the complete story.

In any event, we will not try to compute chiral states in the IR theory, but, we did think it important to demonstrate how in principle the IR rings can be related to the sheaf cohomology groups we discuss in this paper.

\subsection{Pseudo-topological twists}

Theories with $(0,2)$ supersymmetry admit pseudo-topological twists, resulting in theories known as the $\mathrm{A} / 2$ and $\mathrm{B} / 2$ models $[28,31]$. The $\mathrm{A} / 2$ model twist of a $(0,2)$ nonlinear sigma model on a space $X$ with bundle $\mathcal{E}$ is well-defined when, in addition to the Green-Schwarz anomaly cancellation condition,

$$
\operatorname{det} \mathcal{E}^{*} \cong K_{X}
$$

and the $\mathrm{B} / 2$ model twist is well-defined when, in addition to Green-Schwarz,

$$
\operatorname{det} \mathcal{E} \cong K_{X}
$$

Dualizing the bundle $\mathcal{E}$ yields an isomorphic quantum field theory, in which the $\mathrm{A} / 2$ and $\mathrm{B} / 2$ twists are exchanged. 
In principle, if the bundle $\mathcal{E}$ is reducible, then there are further variants, further topological twists, obtained by dualizing the various individual factors. Dualizing those bundle factors produces an isomorphic quantum field theory, but modifies the twists. If we think of the twists as twisting along a U(1) symmetry of the theory, then the point here is that if the bundle is reducible, then there are additional $U(1)$ symmetries (corresponding to different phase factors on different factors) which yield different pseudo-topological twists, or equivalently, the $\mathrm{A} / 2$ and $\mathrm{B} / 2$ twist but for a bundle obtained by dualizing some of the factors. We will speak of a theory 'admitting an $\mathrm{A} / 2$ or $\mathrm{B} / 2$ twist' when the particular choice of $\mathcal{E}$ satisfies one of the conditions above.

Now, let us turn to the examples appearing in triality. As a warm-up, consider a $(0,2)$ nonlinear sigma model on the Grassmannian $G(k, n)$ with bundle

$$
\mathcal{E}=S^{\oplus A} \oplus\left(Q^{*}\right)^{\oplus(2 k+A-n)} \oplus\left(\operatorname{det} S^{*}\right)^{\oplus 2},
$$

from which we derive

$$
\begin{aligned}
\operatorname{det} \mathcal{E} & \cong(\operatorname{det} S)^{A-2} \otimes\left(\operatorname{det} Q^{*}\right)^{2 k+A-n} \\
& \cong(\operatorname{det} S)^{2 A+2 k-n-2}
\end{aligned}
$$

using $\operatorname{det} Q^{*} \cong \operatorname{det} S$. For the tangent bundle,

$$
0 \longrightarrow S^{*} \otimes S \longrightarrow S^{*} \otimes \mathcal{O}^{n} \longrightarrow T \longrightarrow 0,
$$

hence $K_{X} \cong(\operatorname{det} S)^{n}$. Putting this together, we find that this model will admit an $\mathrm{A} / 2$ twist when

$$
0=A+k-1
$$

and the same model will admit a $\mathrm{B} / 2$ twist when

$$
n=A+k-1 \text {. }
$$

For bundles of this particular form, examples admitting an $\mathrm{A} / 2$ twist will be rather rare, as it requires $A+k=1$, but models admitting a $\mathrm{B} / 2$ twist are less uncommon.

Next, let us take advantage of the fact that $\mathcal{E}$ is reducible. If we dualize the middle and last factors, we get the bundle

$$
\mathcal{E}^{\prime}=S^{\oplus A} \oplus(Q)^{\oplus(2 k+A-n)} \oplus(\operatorname{det} S)^{\oplus 2},
$$

for which we compute

$$
\operatorname{det} \mathcal{E}^{\prime} \cong(\operatorname{det} S)^{A+2} \otimes(\operatorname{det} Q)^{2 k+A-n} \cong(\operatorname{det} Q)^{2 k-n-2},
$$

and so

$$
\left(\operatorname{det} \mathcal{E}^{\prime}\right)^{-1} \otimes K_{X} \cong(\operatorname{det} S)^{2 k-n-2} \otimes(\operatorname{det} S)^{n} \cong(\operatorname{det} S)^{2 k-2} .
$$

Clearly, if $k=1$, then this presentation admits a B/2 twist. However, if we are willing to make the global symmetry rotating $Q^{*}$ 's more obscure and dualize pairs of them individually, then we can build an alternative bundle $\mathcal{E}^{\prime \prime}$ which admits a $\mathrm{B} / 2$ twist.

Thus, by suitably dualizing gauge bundle factors, we can find a B/2 twist of any $(0,2)$ theory related by triality. That said, a given $\mathrm{B} / 2$ twist is not invariant under triality, as even dualizing a bundle will replace the original $\mathrm{B} / 2$ twist with something different. 


\subsection{First example}

In this section we will compare chiral states in examples of different UV NLSM's that are related by triality - some as different phases of the same GLSM, others from different GLSM's. We will find in the examples we compute that all the different presentations have some states in common, and a few states that differ between presentations. However, the states that are in common, all are defined by integrable representations of the global symmetry groups, integrable with respect to the proposed IR affine algebras. Furthermore, the mismatched states will not contribute to elliptic genera and are defined by nonintegrable representations, strongly suggesting that they become massive along the RG flow, indirectly verifying the triality proposal, and also giving a very clean example of how non-protected operators can change along RG flow.

\subsubsection{First GLSM}

We shall begin with a computation of chiral states in the two phases of a $(0,2)$ GLSM pertinent to triality. Let's take $k=1, A=3$, and $n=3$, so $2 k+A-n=2$. The two phases are defined by

$$
\mathcal{E} \equiv U \otimes S+V \otimes Q^{*}+W \otimes \operatorname{det} S^{*} \longrightarrow \mathbb{P}^{2}=\mathbb{P} \tilde{V}^{*}
$$

for $r \gg 0$, and

$$
\mathcal{F} \equiv U \otimes S^{*}+\tilde{V} \otimes Q^{*}+W \otimes \operatorname{det} S \longrightarrow \mathbb{P}^{1}=\mathbb{P} V^{*}
$$

for $r \ll 0$, where in both phases,

$$
U=\mathbb{C}^{3}, \quad V=\mathbb{C}^{2}, \quad W=\mathbb{C}^{2}, \quad \tilde{V}=\mathbb{C}^{3} .
$$

(For brevity, we only list the underlying geometries, rather than the full matter content of each GLSM. It may be worth observing that although two of the triality phases are given by abelian GLSM's, the third is given by a GLSM with gauge group U(2).)

According to triality, these two nonlinear sigma models should flow in the IR to the same point, hence, on the face of it, one would expect them to have isomorphic chiral states.

It is straightforward to check that, in both cases, anomaly cancellation holds, and furthermore each phase admits a $\mathrm{B} / 2$ twist, so that the Fock vacuum line bundle is trivial. As remarked earlier, for our purposes it is important to give a precise presentation of the Fock vacuum line bundle in terms of powers of $S^{*}$ and $Q^{*}$, and in both phases we will present it as the canonical trivial bundle, i.e. $K_{(0)} S^{*} \otimes K_{(0, \cdots, 0)} Q^{*}$ in the notation of appendix A. Hence we can compute the chiral states in the form

$$
H^{\bullet}(X, \wedge \bullet \mathcal{E}) \text {. }
$$

Each phase has a set of global nonanomalous (chiral) $\mathrm{U}(1)^{3}$ symmetries, which are given by

\begin{tabular}{|l|cccc|}
\hline & $\tilde{V}$ & $U$ & $V$ & $W$ \\
\hline $\mathrm{U}(1)_{(1)}$ & 0 & 0 & -1 & -1 \\
$\mathrm{U}(1)_{(2)}$ & 1 & 0 & 0 & $-3 / 2$ \\
$\mathrm{U}(1)_{(3)}$ & 0 & 1 & 0 & $+3 / 2$ \\
\hline
\end{tabular}


In a slight variation from $[24,25]$, we have assigned the same charge to all elements of $W$, for simplicity in comparing states.

For the purposes of correctly comparing symmetries, it is useful to distinguish $\mathbb{P} V$ from $\mathbb{P} V^{*}$, for example. Briefly, on the space $\mathbb{P} V$, the homogeneous coordinates naturally transform under $V^{*}$. On that space,

$$
0 \longrightarrow S \longrightarrow V \otimes \mathcal{O} \longrightarrow Q \longrightarrow 0
$$

so dualizing, taking the long exact sequence, and using $H^{\bullet}\left(Q^{*}\right)=0$, we find that the homogeneous coordinates are given by

$$
H^{0}\left(S^{*}\right)=H^{0}\left(V^{*} \otimes \mathcal{O}\right)=V^{*}
$$

Here, for $r \gg 0$, the homogeneous coordinates naturally transform under $\tilde{V}$, and for $r \ll 0$, $V$, hence the two geometries are naturally $\mathbb{P} \tilde{V}^{*}$ and $\mathbb{P} V^{*}$, respectively.

In the remaining tables in this section we list all of the states in the two phases, beginning with all of the matching states. Now, to match states in principle we need only match representations of nonanomalous symmetries. However, in this example, the bulk of the matching states match full (anomalous) $G L(U) \times G L(V) \otimes G L(W) \otimes G L(\tilde{V})$ representations. In table 1 we list all such states which match exactly, as representations of the anomalous symmetry above, between the two phases. The state column lists the representation of

$$
G L(U) \times G L(V) \times G L(W) \times G L(\tilde{V})
$$

obtained from the Bott-Borel-Weil computation. As overall $\mathbb{C}^{\times}$factors are individually anomalous, we separately list the nonanomalous

$$
\mathrm{SL}(U) \times \mathrm{SL}(V) \times \mathrm{SL}(W) \times \mathrm{SL}(\tilde{V})=\mathrm{SU}(3) \times \mathrm{SU}(2) \times \mathrm{SU}(2) \times \mathrm{SU}(3)
$$

representations and nonanomalous global

$$
\mathrm{U}(1)_{(1)} \times \mathrm{U}(1)_{(2)} \times \mathrm{U}(1)_{(3)}
$$

charges in the last two columns. Immediately after the state listing, the next two columns list in which wedge power of $\mathcal{E}$ the state was obtained, and the cohomological degree, respectively, in the $r \gg 0$ phase, and the next two after that give the same information for the $r \ll 0$ phase.

The $\mathrm{U}(1)^{3}$ charges listed include the fractional charges from the Fock vacua. For the $r \gg 0$ phase, for example, the first and third U(1)'s act linearly on the left-moving fermions, and so the fractional charges can be computed directly using standard ${ }^{8}$ fractional fermion

${ }^{8}$ Alternatively, they can also be computed from our expression for the Fock vacuum line bundle

$$
(\operatorname{det} \mathcal{E})^{-1 / 2} \otimes K_{X}^{+1 / 2}
$$

For the $r \gg 0$ phase, where

$$
\mathcal{E}=U \otimes S+V \otimes Q^{*}+W \otimes S^{*} \longrightarrow G\left(1, \tilde{V}^{*}\right)
$$


computations. The second $\mathrm{U}(1)$ does not act linearly on the right-moving fermions, and so the corresponding vacuum charge is a bit more subtle to compute. We computed it as $-1 / 2$ of the charge of the top-degree state, the Serre dual to the state in $\wedge^{0} \mathcal{E}$. This guaranteed that the first and last entries in table 1 have opposite charges. In any event, the result is that for both the $r \gg 0$ and $r \ll 0$ phases, the fractional $\mathrm{U}(1)^{3}$ charge of the vacuum was taken to be $(+3,0,-3)$. It is a highly nontrivial consistency check that, with that choice, all other states related by Serre duality also have opposite charges.

As a consistency check, note that all of the states in all of the tables in this section come in Serre-dual pairs, exchanging not only cohomology degrees but also dualizing representations and $\mathrm{U}(1)^{3}$ charges. (Recall that in $\mathrm{SU}(2), \mathbf{2}=\overline{\mathbf{2}}$, so the dualization only acts nontrivially on $\mathrm{SU}(3)$ representations.)

A few additional states have matching representations of anomaly-free global symmetries, i.e. the same $\mathrm{SU}(3) \times \cdots$ representations and $\mathrm{U}(1)^{3}$ charges, but are expressed differently in terms of anomalous representations. These are listed in table 2.

Finally, there are a few remaining states in each phase that do not match any states in the other phase at all, listed in table 3. Note in particular that the states in neither phase are a proper subset of the states in the other: both phases have states not in the other.

To aid the reader, as the methods are not commonly used in the physics community, let us take a moment to illustrate how, for example, the next-to-last entry in table 1 was computed, in the $r \gg 0$ phase. This entry arose as the only nonzero contribution to $H^{\bullet}\left(\mathbb{P}^{2}, \wedge^{8} \mathcal{E}\right)$. Now,

$$
\begin{aligned}
\wedge^{8} \mathcal{E}=\wedge^{8}(U \otimes S & \left.+V \otimes Q^{*}+W \otimes S^{*}\right) \\
=\wedge^{2}(U \otimes & S) \otimes \wedge^{4}\left(V \otimes Q^{*}\right) \otimes \wedge^{2}\left(W \otimes S^{*}\right) \\
& +\wedge^{3}(U \otimes S) \otimes \wedge^{3}\left(V \otimes Q^{*}\right) \otimes \wedge^{2}\left(W \otimes S^{*}\right) \\
& +\wedge^{3}(U \otimes S) \otimes \wedge^{4}\left(V \otimes Q^{*}\right) \otimes \wedge^{1}\left(W \otimes S^{*}\right),
\end{aligned}
$$

since $U \otimes S$ has rank $3, V \otimes Q^{*}$ has rank 4, and $W \otimes S^{*}$ has rank 2. (More generally, we sum over all combinations of wedge powers adding up to the given power, which in this case is eight.) To compute each of the wedge powers appearing, we use the identity

$$
\wedge^{r}(A \otimes B)=\sum_{|\lambda|=r} K_{\lambda} A \otimes K_{\lambda^{T}} B
$$

where the sum is over Young diagrams $\lambda$ with $r$ boxes, and $K_{\lambda} A$ denotes a tensor product of $A$ 's determined by the Young diagram, for example

$$
K_{\square} A=\operatorname{Sym}^{2} A, \quad K_{\boxminus} A=\wedge^{2} A .
$$

it is straightforward to compute that

$$
(\operatorname{det} \mathcal{E})^{-1 / 2} \otimes K_{X}^{+1 / 2}=\left(\wedge^{3} U\right)^{-1 / 2} \otimes\left(\wedge^{2} V\right)^{-1} \otimes\left(\wedge^{2} W\right)^{-1 / 2} \otimes\left(\wedge^{3} \tilde{V}^{*}\right)^{-1 / 2},
$$

for which the first and third U(1) charges are computed to match those one would obtain by standard fractional fermion techniques. We caution against applying the same method for the fractional charge under the second $\mathrm{U}(1)$ in the $r \gg 0$ phase, as its action is not linear on the right-moving fermions. The method above will sometimes give the correct result in that case, but will also often not. 


\begin{tabular}{|c|c|c|c|c|c|c|}
\hline \multirow[b]{2}{*}{ State } & \multicolumn{2}{|c|}{$r \gg 0$} & \multicolumn{2}{|c|}{$r \ll 0$} & \multirow[b]{2}{*}{ Rep' } & \multirow[b]{2}{*}{$\mathrm{U}(1)^{3}$} \\
\hline & $\wedge^{\bullet} \mathcal{E}$ & $H^{\bullet}\left(\mathbb{P}^{2}\right)$ & $\wedge \cdot \mathcal{F}$ & $H^{\bullet}\left(\mathbb{P}^{1}\right)$ & & \\
\hline 1 & 0 & 0 & 0 & 0 & $(\mathbf{1}, \mathbf{1}, \mathbf{1}, \mathbf{1})$ & $(+3,0,-3)$ \\
\hline$W \otimes \tilde{V}$ & 1 & 0 & 2 & 1 & $(\mathbf{1}, \mathbf{1}, \mathbf{2}, \mathbf{3})$ & $(+2,-1 / 2,-3 / 2)$ \\
\hline$\wedge^{2} V \otimes \wedge^{2} \tilde{V}$ & 2 & 1 & 2 & 1 & $(\mathbf{1}, \mathbf{1}, \mathbf{1}, \overline{\mathbf{3}})$ & $(+1,+2,-3)$ \\
\hline$U \otimes V$ & 2 & 1 & 1 & 0 & $(3,2,1,1)$ & $(+2,0,-2)$ \\
\hline$U \otimes W$ & 2 & 0 & 2 & 0 & $(3,1,2,1)$ & $(+2,-3 / 2,-1 / 2)$ \\
\hline$V \otimes W \otimes \wedge^{2} \tilde{V}$ & 2 & 0 & 3 & 1 & $(\mathbf{1}, 2,2, \overline{3})$ & $(+1,+1 / 2,-3 / 2)$ \\
\hline$U \otimes \wedge^{2} V \otimes \tilde{V}$ & 3 & 1 & 2 & 0 & $(3, \mathbf{1}, \mathbf{1}, \mathbf{3})$ & $(+1,+1,-2)$ \\
\hline$U \otimes \wedge^{2} W \otimes \tilde{V}$ & 3 & 0 & 4 & 1 & $(3,1,1,3)$ & $(+1,-2,+1)$ \\
\hline$V \otimes \wedge^{2} V \otimes \wedge^{3} \tilde{V}$ & 3 & 1 & 3 & 1 & $(\mathbf{1}, \mathbf{2}, \mathbf{1}, \mathbf{1})$ & $(0,+3,-3)$ \\
\hline $\operatorname{Sym}^{2} V \otimes W \otimes \wedge^{3} \tilde{V}$ & 3 & 0 & 4 & 1 & $(\mathbf{1}, \mathbf{3}, \mathbf{2}, \mathbf{1})$ & $(0,+3 / 2,-3 / 2)$ \\
\hline$\wedge^{2} U \otimes \operatorname{Sym}^{2} V$ & 4 & 2 & 2 & 0 & $(\overline{\mathbf{3}}, \mathbf{3}, \mathbf{1}, \mathbf{1})$ & $(+1,0,-1)$ \\
\hline$\wedge^{2} U \otimes V \otimes W$ & 4 & 1 & 3 & 0 & $(\overline{3}, 2,2,1)$ & $(+1,-3 / 2,+1 / 2)$ \\
\hline$\wedge^{2} U \otimes \wedge^{2} W$ & 4 & 0 & 4 & 0 & $(\overline{\mathbf{3}}, \mathbf{1}, \mathbf{1}, \mathbf{1})$ & $(+1,-3,+2)$ \\
\hline$U \otimes \wedge^{2} V \otimes W \otimes \wedge^{2} \tilde{V}$ & 4 & 1 & 4 & 1 & $(3,1,2, \overline{3})$ & $(0,+1 / 2,-1 / 2)$ \\
\hline$U \otimes V \otimes \wedge^{2} W \otimes \wedge^{2} \tilde{V}$ & 4 & 0 & 5 & 1 & $(3,2,1, \overline{3})$ & $(0,-1,+1)$ \\
\hline$\wedge^{2} U \otimes \wedge^{2} V \otimes V \otimes \tilde{V}$ & 5 & 2 & 3 & 0 & $(\overline{3}, 2,1,3)$ & $(0,+1,-1)$ \\
\hline$\wedge^{2} U \otimes W \otimes \wedge^{2} V \otimes \tilde{V}$ & 5 & 1 & 4 & 0 & $(\overline{3}, \mathbf{1}, \mathbf{2}, \mathbf{3})$ & $(0,-1 / 2,+1 / 2)$ \\
\hline$U \otimes\left(\wedge^{2} V\right)^{2} \otimes \wedge^{3} \tilde{V}$ & 5 & 2 & 4 & 1 & $(3,1,1,1)$ & $(-1,+3,-2)$ \\
\hline$U \otimes W \otimes \wedge^{2} V \otimes V \otimes \wedge^{3} \tilde{V}$ & 5 & 1 & 5 & 1 & $(3,2,2,1)$ & $(-1,+3 / 2,-1 / 2)$ \\
\hline$U \otimes \operatorname{Sym}^{2} V \otimes \wedge^{2} W \otimes \wedge^{3} \tilde{V}$ & 5 & 0 & 6 & 1 & $(\mathbf{3}, \mathbf{3}, \mathbf{1}, \mathbf{1})$ & $(-1,0,+1)$ \\
\hline$\wedge^{3} U \otimes \operatorname{Sym}^{2} V \otimes W$ & 6 & 2 & 4 & 0 & $(\mathbf{1}, \mathbf{3}, \mathbf{2}, \mathbf{1})$ & $(0,-3 / 2,+3 / 2)$ \\
\hline$\wedge^{3} U \otimes V \otimes \wedge^{2} W$ & 6 & 1 & 5 & 0 & $(1,2,1,1)$ & $(0,-3,+3)$ \\
\hline$\wedge^{2} U \otimes\left(\wedge^{2} V\right)^{2} \otimes \wedge^{2} \tilde{V}$ & 6 & 2 & 4 & 0 & $(\overline{\mathbf{3}}, \mathbf{1}, \mathbf{1}, \overline{\mathbf{3}})$ & $(-1,+2,-1)$ \\
\hline$\wedge^{2} U \otimes \wedge^{2} V \otimes \wedge^{2} W \otimes \wedge^{2} \tilde{V}$ & 6 & 1 & 6 & 1 & $(\overline{\mathbf{3}}, \mathbf{1}, \mathbf{1}, \overline{\mathbf{3}})$ & $(-1,-1,+2)$ \\
\hline$\wedge^{3} U \otimes \wedge^{2} V \otimes V \otimes W \otimes \tilde{V}$ & 7 & 2 & 5 & 0 & $(1,2,2,3)$ & $(-1,-1 / 2,+3 / 2)$ \\
\hline$\wedge^{2} U \otimes\left(\wedge^{2} V\right)^{2} \otimes W \otimes \wedge^{3} \tilde{V}$ & 7 & 2 & 6 & 1 & $(\overline{3}, \mathbf{1}, \mathbf{2}, \mathbf{1})$ & $(-2,+3 / 2,+1 / 2)$ \\
\hline$\wedge^{2} U \otimes \wedge^{2} V \otimes V \otimes \wedge^{2} W \otimes \wedge^{3} \tilde{V}$ & 7 & 1 & 7 & 1 & $(\overline{3}, 2,1,1)$ & $(-2,0,+2)$ \\
\hline$\wedge^{3} U \otimes \wedge^{2} V \otimes \wedge^{2} W \otimes \tilde{V}$ & 7 & 1 & 6 & 0 & $(\mathbf{1}, \mathbf{1}, \mathbf{1}, \mathbf{3})$ & $(-1,-2,+3)$ \\
\hline$\wedge^{3} U \otimes\left(\wedge^{2} V\right)^{2} \otimes W \otimes \wedge^{2} \tilde{V}$ & 8 & 2 & 6 & 0 & $(\mathbf{1}, \mathbf{1}, \mathbf{2}, \overline{\mathbf{3}})$ & $(-2,+1 / 2,+3 / 2)$ \\
\hline$\wedge^{3} U \otimes\left(\wedge^{2} V\right)^{2} \otimes \wedge^{2} W \otimes \wedge^{3} \tilde{V}$ & 9 & 2 & 8 & 1 & $(\mathbf{1}, \mathbf{1}, \mathbf{1}, \mathbf{1})$ & $(-3,0,+3)$ \\
\hline
\end{tabular}

Table 1. List of states shared between the two phases.

\begin{tabular}{|c|c|c|c|}
\hline $\begin{array}{c}r \gg 0 \\
\text { State, } \wedge^{\bullet} \mathcal{E}, H^{\bullet}\left(\mathbb{P}^{2}\right)\end{array}$ & $\begin{array}{c}r \ll 0 \\
\text { State, } \wedge^{\bullet} \mathcal{F}, H^{\bullet}\left(\mathbb{P}^{1}\right)\end{array}$ & Rep' & $\mathrm{U}(1)^{3}$ \\
\hline$\wedge^{3} U \otimes \wedge^{3} \tilde{V}^{*}, 3,2$ & $\wedge^{2} W \otimes \wedge^{2} V^{*}, 2,1$ & $(\mathbf{1}, \mathbf{1}, \mathbf{1}, \mathbf{1})$ & $(+3,-3,0)$ \\
\hline$\wedge^{3} U \otimes V \otimes \wedge^{2} \tilde{V}^{*}, 4,2$ & $\tilde{V} \otimes \wedge^{2} W \otimes V^{*}, 3,1$ & $(\mathbf{1}, 2, \mathbf{1}, 3)$ & $(+2,-2,0)$ \\
\hline $\operatorname{Sym}^{2} V \otimes \wedge^{2} W \otimes \tilde{V} \otimes \wedge^{3} \tilde{V}, 4,0$ & $\wedge^{3} U \otimes \tilde{V} \otimes \wedge^{2} V \otimes \operatorname{Sym}^{2} V, 4,0$ & $(\mathbf{1}, \mathbf{3}, \mathbf{1}, \mathbf{3})$ & $(-1,+1,0)$ \\
\hline$\wedge^{3} U \otimes \operatorname{Sym}^{2} V \otimes \wedge^{3} \tilde{V}^{*} \otimes \wedge^{2} \tilde{V}, 5,2$ & $\wedge^{2} \tilde{V} \otimes \wedge^{2} W \otimes \wedge^{2} V^{*} \otimes \operatorname{Sym}^{2} V, 4,1$ & $(\mathbf{1}, \mathbf{3}, \mathbf{1}, \overline{\mathbf{3}})$ & $(+1,-1,0)$ \\
\hline$\wedge^{2} V \otimes V \otimes \wedge^{2} W \otimes \wedge^{3} \tilde{V} \otimes \wedge^{2} \tilde{V}, 5,0$ & $\wedge^{3} U \otimes \wedge^{2} \tilde{V} \otimes\left(\wedge^{2} V\right)^{2} \otimes V, 5,0$ & $(\mathbf{1}, \mathbf{2}, \mathbf{1}, \overline{\mathbf{3}})$ & $(-2,+2,0)$ \\
\hline$\left(\wedge^{2} V\right)^{2} \otimes \wedge^{2} W \otimes\left(\wedge^{3} \tilde{V}\right)^{2}, 6,0$ & $\wedge^{3} U \otimes \wedge^{3} \tilde{V} \otimes\left(\wedge^{2} V\right)^{3}, 6,0$ & $(\mathbf{1}, \mathbf{1}, \mathbf{1}, \mathbf{1})$ & $(-3,+3,0)$ \\
\hline
\end{tabular}

Table 2. Additional shared states defined by matching representations of anomaly-free global symmetries. 


\begin{tabular}{|c|c|c|c|c|c|c|}
\hline \multirow[b]{2}{*}{ State } & \multicolumn{2}{|c|}{$r \gg 0$} & \multicolumn{2}{|c|}{$r \ll 0$} & \multirow[b]{2}{*}{ Rep' } & \multirow[b]{2}{*}{$\mathrm{U}(1)^{3}$} \\
\hline & $\wedge \bullet \mathcal{E}$ & $H^{\bullet}\left(\mathbb{P}^{2}\right)$ & $\wedge \cdot \mathcal{F}$ & $H^{\bullet}\left(\mathbb{P}^{1}\right)$ & & \\
\hline$\wedge^{2} W \otimes \operatorname{Sym}^{2} \tilde{V}$ & 2 & 0 & - & - & $(1,1,1,6)$ & $(+1,-1,0)$ \\
\hline$V \otimes \wedge^{2} W \otimes K_{(2,1,0)} \tilde{V}$ & 3 & 0 & - & - & $(1,2,1,8)$ & $(0,0,0)$ \\
\hline$\wedge^{2} V \otimes \wedge^{2} W \otimes K_{(2,2,0)} \tilde{V}$ & 4 & 0 & - & - & $(\mathbf{1}, \mathbf{1}, \mathbf{1}, \mathbf{6})$ & $(-1,+1,0)$ \\
\hline$\wedge^{3} U \otimes \wedge^{2} V \otimes \wedge^{3} \tilde{V}^{*} \otimes \operatorname{Sym}^{2} \tilde{V}$ & 5 & 2 & - & - & $(\mathbf{1}, \mathbf{1}, \mathbf{1}, \mathbf{6})$ & $(+1,-1,0)$ \\
\hline$\wedge^{3} U \otimes K_{(2,1)} V \otimes K_{(1,0,-1)} \tilde{V}$ & 6 & 2 & - & - & $(1,2,1,8)$ & $(0,0,0)$ \\
\hline$\wedge^{3} U \otimes\left(\wedge^{2} V\right)^{2} \otimes \wedge^{3} \tilde{V} \otimes \operatorname{Sym}^{2} \tilde{V}^{*}$ & 7 & 2 & - & - & $(\mathbf{1}, \mathbf{1}, \mathbf{1}, \mathbf{6})$ & $(-1,+1,0)$ \\
\hline$\wedge^{3} U \otimes \operatorname{Sym}^{3} V$ & - & - & 3 & 0 & $(\mathbf{1}, 4,1,1)$ & $(0,0,0)$ \\
\hline$\wedge^{3} \tilde{V} \otimes \wedge^{2} W \otimes \wedge^{2} V^{*} \otimes \operatorname{Sym}^{3} V$ & - & - & 5 & 1 & $(\mathbf{1}, \mathbf{4}, \mathbf{1}, \mathbf{1})$ & $(0,0,0)$ \\
\hline
\end{tabular}

Table 3. List of all states which are not shared between the two phases.

Thus, for example,

$$
\begin{aligned}
\wedge^{2}\left(W \otimes S^{*}\right) & =K_{\square} W \otimes K_{\square} S^{*}+K_{\square} W \otimes K_{\square} S^{*}, \\
& =\operatorname{Sym}^{2} W \otimes \wedge^{2} S^{*}+\wedge^{2} W \otimes \operatorname{Sym}^{2} S^{*} .
\end{aligned}
$$

However, since $S^{*}$ has rank one, $\wedge^{2} S^{*}=0$, and so

$$
\wedge^{2}\left(W \otimes S^{*}\right)=\wedge^{2} W \otimes \operatorname{Sym}^{2} S^{*} .
$$

Similarly,

$$
\wedge^{3}\left(V \otimes Q^{*}\right)=K_{\square} V \otimes K_{\square} Q^{*}, \quad \wedge^{4}\left(V \otimes Q^{*}\right)=K_{\square} V \otimes K_{\boxminus} Q^{*},
$$

since both $V$ and $Q^{*}$ have rank 2, eliminating most possible contributions, and

$$
\wedge^{2}(U \otimes S)=\wedge^{2} U \otimes \operatorname{Sym}^{2} S, \wedge^{3}(U \otimes S)=\wedge^{3} U \otimes \operatorname{Sym}^{3} S,
$$

since $S$ has rank 1, eliminating most possible contributions. Putting this together, we find

$$
\begin{aligned}
\wedge^{8} \mathcal{E}=\wedge^{2} U \otimes & K_{\varpi} V \otimes \wedge^{2} W \otimes \operatorname{Sym}^{2} S \otimes K_{\square} Q^{*} \otimes \operatorname{Sym}^{2} S^{*} \\
& +\wedge^{3} U \otimes K_{\square} V \otimes \wedge^{2} W \otimes \operatorname{Sym}^{3} S \otimes K_{\square} Q^{*} \otimes \operatorname{Sym}^{2} S^{*} \\
& +\wedge^{3} U \otimes K_{\square} V \otimes W \otimes \operatorname{Sym}^{3} S \otimes K_{\square} Q^{*} \otimes S^{*} .
\end{aligned}
$$

Thus,

$$
\begin{aligned}
H^{\bullet}\left(\mathbb{P}^{2}, \wedge^{8} \mathcal{E}\right)=\wedge^{2} U \otimes & K_{\boxplus} V \otimes \wedge^{2} W \otimes H^{\bullet}\left(\mathbb{P}^{2}, K_{(2,2)} Q^{*}\right) \\
& +\wedge^{3} U \otimes K_{\square} V \otimes \wedge^{2} W \otimes H^{\bullet}\left(\mathbb{P}^{2}, K_{(-1)} S^{*} \otimes K_{(2,1)} Q^{*}\right) \\
& +\wedge^{3} U \otimes K_{\square} V \otimes W \otimes H^{\bullet}\left(\mathbb{P}^{2}, K_{(-2)} S^{*} \otimes K_{(2,2)} Q^{*}\right),
\end{aligned}
$$

and from Bott-Borel-Weil,

$$
\begin{aligned}
H^{\bullet}\left(\mathbb{P}^{2}, K_{(2,2)} Q^{*}\right) & =0=H^{\bullet}\left(\mathbb{P}^{2}, K_{(-1)} S^{*} \otimes K_{(2,1)} Q^{*}\right), \\
H^{\bullet}\left(\mathbb{P}^{2}, K_{(-2)} S^{*} \otimes K_{(2,2)} Q^{*}\right) & =K_{(1,1,0)} \tilde{V} \delta^{\bullet, 2} .
\end{aligned}
$$

The next-to-last entry in table 1 follows. 
Note that the states in table 3 might not make a net contribution to elliptic genera. From [9, section 2.1], since this theory is B/2-twistable, the leading term in any refined NLSM elliptic genus will be of the form

$$
(-)^{r / 2} q^{+(r-n) / 12} \sum_{s=0}^{r}(-)^{s} \chi_{y}\left(\wedge^{s} \mathcal{E}\right),
$$

where $y$ represents the refinement by any nonanomalous symmetry, $r$ is the rank of $\mathcal{E}$, and $n$ is the dimension of the base space. Since in our example the rank is greater than the dimension of the base, the unrefined elliptic genus will vanish [9]. However, since there are a number of nonanomalous symmetries, the elliptic genus can be refined, and it is straightforward to check that by adding suitable refinements, the elliptic genus can certainly be made nonzero.

Now, the contribution of any state to the weighted sum (3.1) is weighted in part by a sign determined by the wedge power of the bundle and the degree of the cohomology group in which the state appears. However, all of the mismatched states in table 3 come in pairs with matching representations of nonanomalous symmetries but different signs, and so cancel out. Consider as a prototypical example the two states in the $r \ll 0$ phase. They both live in the same representation $(\mathbf{1}, \mathbf{4}, \mathbf{1}, \mathbf{1})$, and have the same $\mathrm{U}(1)^{3}$ charges, but one enters with $\mathrm{a}+$ and the other with $\mathrm{a}-$, and so their net contribution to the leading term of any elliptic genus refined by any of the symmetries listed is necessarily zero. Thus, the mismatched states listed in table 3 make no net contribution to the leading term of any refined elliptic genus we shall consider. Of course, that guarantees neither that higher order contributions will also vanish, nor that there are no other nonanomalous symmetries whose refinements might receive a contribution. However, we do find it to be a suggestive observation, supporting the idea that these (mismatched) states might all pair up and become massive along the RG flow.

Now, let us examine these states from the perspective of the exact IR limit proposed in [25]. In the notation of that reference, in the IR there is an affine ${ }^{9}$

$$
\mathrm{SU}(U)_{1} \times \mathrm{SU}(V)_{2} \times \mathrm{SU}(W)_{1} \times \mathrm{SU}(\tilde{V})_{1}=\mathrm{SU}(3)_{1} \times \mathrm{SU}(2)_{2} \times \mathrm{SU}(2)_{1} \times \mathrm{SU}(3)_{1}
$$

symmetry. The corresponding left-chiral states in the IR should be determined in part by integrable representations of the affine symmetry above.

In terms of Young diagrams, a given representation of $\mathrm{SU}(N)_{k}$ is integrable if the Young diagram has no row with more than $k$ boxes [57, section 16.6]. For example, for $k=1$, there are $N$ integrable representations, given by

$$
\mathbf{1}, \mathbf{N}, \wedge^{2} \mathbf{N}, \cdots, \wedge^{N-1} \mathbf{N} .
$$

\footnotetext{
${ }^{9} \mathrm{The} \mathrm{SU}(W)$ affine contribution is not mentioned explicitly in [25]. The level can be computed from the trace anomaly

$$
k_{\Omega}=\operatorname{tr} \gamma^{3} J J=-1 / 2
$$
}

(in their conventions). The corresponding level of the IR current algebra is

$$
2\left|k_{\Omega}\right|=1
$$

and the sign indicates that it is left-moving. 
corresponding to Young diagrams

$$
1, \square, \boxminus, 日, \cdots .
$$

In the case $k=2$, in addition to the $N$ diagrams above, there are $N-1$ diagrams of the form

$$
\square, \boxminus, \boxminus, \boxminus, \cdots,
$$

plus $N-2$ diagrams of the form

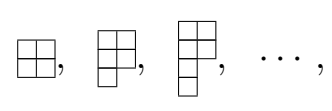

and so forth, for a total of

$$
1+2+\cdots+N-1+N=\frac{1}{2} N(N+1)
$$

integrable representations of $s u(N)$ at level $k=2$.

In the present case, $\mathrm{SU}(3)_{1}$ has integrable representations

\section{$\mathbf{1}, \mathbf{3}, \overline{\mathbf{3}}$,}

$\mathrm{SU}(2)_{1}$ has integrable representations

\section{1,2,}

and $\mathrm{SU}(2)_{2}$ has integrable representations

\section{$1, \quad 2,3$.}

Note that all of the states in tables 1 and 2 have integrable representations of the nonabelian UV global symmetry groups. On the other hand, all of the mismatched states in table 3 have at least one non-integrable representation. If any of the mismatched states survived to the IR, they would then appear to contradict the assertion of [25] that these theories have a nontrivial IR fixed point of the form described there. However, given that their contributions to refined elliptic genera vanish, we find it much more likely that they become massive, leaving only states which are both common across presentations and defined by suitable representations. In this fashion, we have an indirect test of triality.

\subsubsection{Other GLSMs}

In the previous subsection we analyzed the chiral states in the two geometric phases of one of three GLSMs that is believed to flow to a single fixed point. Next we shall repeat the same analysis for another GLSM related to the first by triality. We shall find an analogous structure - states in integrable representations match between the phases, and moreover, we shall see that the states that match between phases also match between GLSMs.

The other two GLSMs can be obtained by cyclically permuting

$$
U \longrightarrow V \longrightarrow \tilde{V}^{*} \longrightarrow U \longrightarrow \cdots
$$


The three large-radius phases correspond to the bundles and spaces given by

$$
\begin{aligned}
& (1): U \otimes S+V \otimes Q^{*}+W \otimes \operatorname{det} S^{*} \longrightarrow G\left(1, \tilde{V}^{*}\right), \\
& (2): V \otimes S+\tilde{V}^{*} \otimes Q^{*}+W \otimes \operatorname{det} S^{*} \longrightarrow G(2, U), \\
& (3): \tilde{V}^{*} \otimes S+U \otimes Q^{*}+W \otimes \operatorname{det} S^{*} \longrightarrow G(1, V),
\end{aligned}
$$

and the three $r \ll 0$ phases are described by

$$
\begin{aligned}
& (1): U \otimes S^{*}+\tilde{V} \otimes Q^{*}+W \otimes \operatorname{det} S \longrightarrow G\left(1, V^{*}\right), \\
& (2): V \otimes S^{*}+U^{*} \otimes Q^{*}+W \otimes \operatorname{det} S \longrightarrow G(2, \tilde{V}), \\
& (3): \tilde{V}^{*} \otimes S^{*}+V^{*} \otimes Q^{*}+W \otimes \operatorname{det} S \longrightarrow G\left(1, U^{*}\right) .
\end{aligned}
$$

(As a consistency check, the $\mathrm{U}(1)^{3}$ global symmetry is nonanomalous in each of the six phases above.) In the previous section, we computed the chiral rings in both phases of GLSM (1), and note that the $r \ll 0$ phase of (1) is closely related by mathematical duality to the $r \gg 0$ phase of (3). The remaining geometry can be described as either the $r \gg 0$ phase of (2) or the $r \ll 0$ phase of (3). Let us therefore focus on GLSM (3), and compute the chiral rings in the two NLSM phases.

The reader should note that the phases of GLSM (3) are closely related to those of (1). Specifically, we can get the $r \ll 0$ phase of (3) from the $r \gg 0$ phase of (1), and the $r \gg 0$ phase of (3) from the $r \ll 0$ phase of (1), by making the substitutions

$$
U \leftrightarrow \tilde{V}, \quad V \leftrightarrow V^{*}, \quad W \leftrightarrow W^{*},
$$

so rather than re-compute spectra from scratch, we can simply re-use the existing tables of states by making the replacements above.

The $\mathrm{U}(1)^{3}$ charges are computed from a slightly different action than in the first GLSM, given by

\begin{tabular}{|c|cccc|}
\hline & $\tilde{V}$ & $U$ & $V$ & $W$ \\
\hline $\mathrm{U}(1)_{(1)}$ & 0 & 0 & +1 & +1 \\
$\mathrm{U}(1)_{(2)}$ & 1 & 0 & 0 & $-3 / 2$ \\
$\mathrm{U}(1)_{(3)}$ & 0 & 1 & 0 & $+3 / 2$ \\
\hline
\end{tabular}

(These are almost the same as in the first GLSM, except that there is a sign flip on the charges of the first U(1).) With these (nonanomalous) charge assignments, we shall see that the states we compute in this GLSM which are shared between the two geometric phases, are also shared with the first GLSM. In any event, following the same procedure as in the last section, we compute $(+3,-3,0)$ for both the $r \gg 0$ and $r \ll 0$ phases.

By making the substitutions described above, we can immediately write down the chiral states in the two phases of this GLSM. These states are encoded in tables 4, 5, and 6 , which are precise analogues of the corresponding tables 1,2,3 for the previous GLSM related by triality. As a consistency check, it is straightforward to check that all states come in Serre dual pairs in which representations are dualized. Furthermore, all of 


\begin{tabular}{|c|c|c|c|c|c|c|}
\hline \multirow[b]{2}{*}{ State } & \multicolumn{2}{|c|}{$r \ll 0$} & \multicolumn{2}{|c|}{$r \gg 0$} & \multirow[b]{2}{*}{ Rep' } & \multirow[b]{2}{*}{$\mathrm{U}(1)^{3}$} \\
\hline & $\wedge \bullet \mathcal{E}$ & $H^{\bullet}\left(\mathbb{P}^{2}\right)$ & $\wedge \cdot \mathcal{F}$ & $H^{\bullet}\left(\mathbb{P}^{1}\right)$ & & \\
\hline 1 & 0 & 0 & 0 & 0 & $(1,1,1,1)$ & $(+3,-3,0)$ \\
\hline$W^{*} \otimes U$ & 1 & 0 & 2 & 1 & $(3,1,2,1)$ & $(+2,-3 / 2,-1 / 2)$ \\
\hline$\wedge^{2} V^{*} \otimes \wedge^{2} U$ & 2 & 1 & 2 & 1 & $(\overline{3}, 1,1,1)$ & $(+1,-3,+2)$ \\
\hline$\tilde{V} \otimes V^{*}$ & 2 & 1 & 1 & 0 & $(1,2,1,3)$ & $(+2,-2,0)$ \\
\hline$\tilde{V} \otimes W^{*}$ & 2 & 0 & 2 & 0 & $(1,1,2,3)$ & $(+2,-1 / 2,-3 / 2)$ \\
\hline$V^{*} \otimes W^{*} \otimes \wedge^{2} U$ & 2 & 0 & 3 & 1 & $(\overline{3}, 2,2,1)$ & $(+1,-3 / 2,+1 / 2)$ \\
\hline$\tilde{V} \otimes \wedge^{2} V^{*} \otimes U$ & 3 & 1 & 2 & 0 & $(\mathbf{3}, \mathbf{1}, \mathbf{1}, \mathbf{3})$ & $(+1,-2,+1)$ \\
\hline$\tilde{V} \otimes \wedge^{2} W^{*} \otimes U$ & 3 & 0 & 4 & 1 & $(3,1,1,3)$ & $(+1,+1,-2)$ \\
\hline$V^{*} \otimes \wedge^{2} V^{*} \otimes \wedge^{3} U$ & 3 & 1 & 3 & 1 & $(1,2,1,1)$ & $(0,-3,+3)$ \\
\hline $\operatorname{Sym}^{2} V^{*} \otimes W^{*} \otimes \wedge^{3} U$ & 3 & 0 & 4 & 1 & $(1,3,2,1)$ & $(0,-3 / 2,+3 / 2)$ \\
\hline$\wedge^{2} \tilde{V} \otimes \operatorname{Sym}^{2} V^{*}$ & 4 & 2 & 2 & 0 & $(\mathbf{1}, \mathbf{3}, \mathbf{1}, \overline{\mathbf{3}})$ & $(+1,-1,0)$ \\
\hline$\wedge^{2} \tilde{V} \otimes V^{*} \otimes W^{*}$ & 4 & 1 & 3 & 0 & $(1,2,2, \overline{3})$ & $(+1,+1 / 2,-3 / 2)$ \\
\hline$\wedge^{2} \tilde{V} \otimes \wedge^{2} W^{*}$ & 4 & 0 & 4 & 0 & $(\mathbf{1}, \mathbf{1}, \mathbf{1}, \overline{\mathbf{3}})$ & $(+1,+2,-3)$ \\
\hline$\tilde{V} \otimes \wedge^{2} V^{*} \otimes W^{*} \otimes \wedge^{2} U$ & 4 & 1 & 4 & 1 & $(\overline{\mathbf{3}}, \mathbf{1}, \mathbf{2}, \mathbf{3})$ & $(0,-1 / 2,+1 / 2)$ \\
\hline$\tilde{V} \otimes V^{*} \otimes \wedge^{2} W^{*} \otimes \wedge^{2} U$ & 4 & 0 & 5 & 1 & $(\overline{3}, 2,1,3)$ & $(0,+1,-1)$ \\
\hline$\wedge^{2} \tilde{V} \otimes \wedge^{2} V^{*} \otimes V^{*} \otimes U$ & 5 & 2 & 3 & 0 & $(3,2,1, \overline{3})$ & $(0,-1,+1)$ \\
\hline$\wedge^{2} \tilde{V} \otimes W^{*} \otimes \wedge^{2} V^{*} \otimes U$ & 5 & 1 & 4 & 0 & $(\mathbf{3}, \mathbf{1}, \mathbf{2}, \overline{\mathbf{3}})$ & $(0,+1 / 2,-1 / 2)$ \\
\hline$\tilde{V} \otimes\left(\wedge^{2} V^{*}\right)^{2} \otimes \wedge^{3} U$ & 5 & 2 & 4 & 1 & $(\mathbf{1}, \mathbf{1}, \mathbf{1}, \mathbf{3})$ & $(-1,-2,+3)$ \\
\hline$\tilde{V} \otimes W^{*} \otimes \wedge^{2} V^{*} \otimes V^{*} \otimes \wedge^{3} U$ & 5 & 1 & 5 & 1 & $(1,2,2,3)$ & $(-1,-1 / 2,+3 / 2)$ \\
\hline$\tilde{V} \otimes \operatorname{Sym}^{2} V^{*} \otimes \wedge^{2} W^{*} \otimes \wedge^{3} U$ & 5 & 0 & 6 & 1 & $(1,3,1,3)$ & $(-1,+1,0)$ \\
\hline$\wedge^{3} \tilde{V} \otimes \operatorname{Sym}^{2} V^{*} \otimes W^{*}$ & 6 & 2 & 4 & 0 & $(1,3,2,1)$ & $(0,+3 / 2,-3 / 2)$ \\
\hline$\wedge^{3} \tilde{V} \otimes V^{*} \otimes \wedge^{2} W^{*}$ & 6 & 1 & 5 & 0 & $(1,2,1,1)$ & $(0,+3,-3)$ \\
\hline$\wedge^{2} \tilde{V} \otimes\left(\wedge^{2} V^{*}\right)^{2} \otimes \wedge^{2} U$ & 6 & 2 & 4 & 0 & $(\overline{\mathbf{3}}, \mathbf{1}, \mathbf{1}, \overline{\mathbf{3}})$ & $(-1,-1,+2)$ \\
\hline$\wedge^{2} \tilde{V} \otimes \wedge^{2} V^{*} \otimes \wedge^{2} W^{*} \otimes \wedge^{2} U$ & 6 & 1 & 6 & 1 & $(\overline{\mathbf{3}}, \mathbf{1}, \mathbf{1}, \overline{\mathbf{3}})$ & $(-1,+2,-1)$ \\
\hline$\wedge^{3} \tilde{V} \otimes \wedge^{2} V^{*} \otimes V^{*} \otimes W^{*} \otimes U$ & 7 & 2 & 5 & 0 & $(3,2,2,1)$ & $(-1,+3 / 2,-1 / 2)$ \\
\hline$\wedge^{2} \tilde{V} \otimes\left(\wedge^{2} V\right)^{2} \otimes W^{*} \otimes \wedge^{3} U$ & 7 & 2 & 6 & 1 & $(\mathbf{1}, \mathbf{1}, 2, \overline{3})$ & $(-2,+1 / 2,+3 / 2)$ \\
\hline$\wedge^{2} \tilde{V} \otimes \wedge^{2} V^{*} \otimes V^{*} \otimes \wedge^{2} W^{*} \otimes \wedge^{3} U$ & 7 & 1 & 7 & 1 & $(\mathbf{1}, \mathbf{2}, \mathbf{1}, \overline{\mathbf{3}})$ & $(-2,+2,0)$ \\
\hline$\wedge^{3} \tilde{V} \otimes \wedge^{2} V^{*} \otimes \wedge^{2} W^{*} \otimes U$ & 7 & 1 & 6 & 0 & $(3,1,1,1)$ & $(-1,+3,-2)$ \\
\hline$\wedge^{3} \tilde{V} \otimes\left(\wedge^{2} V^{*}\right)^{2} \otimes W^{*} \otimes \wedge^{2} U$ & 8 & 2 & 6 & 0 & $(\overline{3}, 1,2,1)$ & $(-2,+3 / 2,+1 / 2)$ \\
\hline$\wedge^{3} \tilde{V} \otimes\left(\wedge^{2} V^{*}\right)^{2} \otimes \wedge^{2} W^{*} \otimes \wedge^{3} U$ & 9 & 2 & 8 & 1 & $(1,1,1,1)$ & $(-3,+3,0)$ \\
\hline
\end{tabular}

Table 4. List of states shared between the two phases.

\begin{tabular}{|c|c|c|c|}
\hline $\begin{array}{c}r \ll 0 \\
\text { State, } \wedge^{\bullet} \mathcal{E}, H^{\bullet}\left(\mathbb{P}^{2}\right)\end{array}$ & $\begin{array}{c}r \gg 0 \\
\text { State, } \wedge \bullet \mathcal{F}, H^{\bullet}\left(\mathbb{P}^{1}\right)\end{array}$ & Rep' & $\mathrm{U}(1)^{3}$ \\
\hline$\wedge^{3} \tilde{V} \otimes \wedge^{3} U^{*}, 3,2$ & $\wedge^{2} W^{*} \otimes \wedge^{2} V, 2,1$ & $(1,1,1,1)$ & $(+3,0,-3)$ \\
\hline$\wedge^{3} \tilde{V} \otimes V^{*} \otimes \wedge^{2} U^{*}, 4,2$ & $U \otimes \wedge^{2} W^{*} \otimes V, 3,1$ & $(\mathbf{3}, \mathbf{2}, \mathbf{1}, \mathbf{1})$ & $(+2,0,-2)$ \\
\hline $\operatorname{Sym}^{2} V^{*} \otimes \wedge^{2} W^{*} \otimes K_{(2,1,1)} U, 4,0$ & $\wedge^{3} \tilde{V} \otimes U \otimes K_{(3,1)} V^{*}, 4,0$ & $(\mathbf{3}, \mathbf{3}, \mathbf{1}, \mathbf{1})$ & $(-1,0,+1)$ \\
\hline$\wedge^{3} \tilde{V} \otimes \operatorname{Sym}^{2} V^{*} \otimes K_{(0,0,-1)} U, 5,2$ & $\wedge^{2} U \otimes \wedge^{2} W^{*} \otimes K_{(1,-1)} V, 4,1$ & $(\overline{\mathbf{3}}, \mathbf{3}, \mathbf{1}, \mathbf{1})$ & $(+1,0,-1)$ \\
\hline$K_{(2,1)} V^{*} \otimes \wedge^{2} W^{*} \otimes K_{(2,2,1)} U, 5,0$ & $\wedge^{3} \tilde{V} \otimes \wedge^{2} U \otimes K_{(3,2)} V^{*}, 5,0$ & $(\overline{3}, 2,1,1)$ & $(-2,0,+2)$ \\
\hline$\left(\wedge^{2} V^{*}\right)^{2} \otimes \wedge^{2} W^{*} \otimes\left(\wedge^{3} U\right)^{2}, 6,0$ & $\wedge^{3} \tilde{V} \otimes \wedge^{3} U \otimes\left(\wedge^{2} V^{*}\right)^{3}, 6,0$ & $(\mathbf{1}, \mathbf{1}, \mathbf{1}, \mathbf{1})$ & $(-3,0,+3)$ \\
\hline
\end{tabular}

Table 5. Additional shared states defined by matching representations of anomaly-free global symmetries. 


\begin{tabular}{|c|c|c|c|c|c|c|}
\hline \multirow[b]{2}{*}{ State } & \multicolumn{2}{|c|}{$r \ll 0$} & \multicolumn{2}{|c|}{$r \gg 0$} & \multirow[b]{2}{*}{ Rep' } & \multirow[b]{2}{*}{$\mathrm{U}(1)^{3}$} \\
\hline & $\wedge \bullet \mathcal{E}$ & $H^{\bullet}\left(\mathbb{P}^{2}\right)$ & $\wedge \cdot \mathcal{F}$ & $H^{\bullet}\left(\mathbb{P}^{1}\right)$ & & \\
\hline$\wedge^{2} W^{*} \otimes \operatorname{Sym}^{2} U$ & 2 & 0 & - & - & $(6,1,1,1)$ & $(+1,0,-1)$ \\
\hline$V^{*} \otimes \wedge^{2} W^{*} \otimes K_{(2,1,0)} U$ & 3 & 0 & - & - & $(8,2,1,1)$ & $(0,0,0)$ \\
\hline$\wedge^{2} V^{*} \otimes \wedge^{2} W^{*} \otimes K_{(2,2,0)} U$ & 4 & 0 & - & - & $(6,1,1,1)$ & $(-1,0,+1)$ \\
\hline$\wedge^{3} \tilde{V} \otimes \wedge^{2} V^{*} \otimes \wedge^{3} U^{*} \otimes \operatorname{Sym}^{2} U$ & 5 & 2 & - & - & $(\mathbf{6}, \mathbf{1}, \mathbf{1}, \mathbf{1})$ & $(+1,0,-1)$ \\
\hline$\wedge^{3} \tilde{V} \otimes K_{(2,1)} V^{*} \otimes K_{(1,0,-1)} U$ & 6 & 2 & - & - & $(8,2,1,1)$ & $(0,0,0)$ \\
\hline$\wedge^{3} \tilde{V} \otimes\left(\wedge^{2} V^{*}\right)^{2} \otimes \wedge^{3} U \otimes \operatorname{Sym}^{2} U^{*}$ & 7 & 2 & - & - & $(\mathbf{6}, \mathbf{1}, \mathbf{1}, \mathbf{1})$ & $(-1,0,+1)$ \\
\hline$\wedge^{3} \tilde{V} \otimes \operatorname{Sym}^{3} V^{*}$ & - & - & 3 & 0 & $(\mathbf{1}, \mathbf{4}, \mathbf{1}, \mathbf{1})$ & $(0,0,0)$ \\
\hline$\wedge^{3} U \otimes \wedge^{2} W^{*} \otimes \wedge^{2} V \otimes \operatorname{Sym}^{3} V^{*}$ & - & - & 5 & 1 & $(\mathbf{1}, \mathbf{4}, \mathbf{1}, \mathbf{1})$ & $(0,0,0)$ \\
\hline
\end{tabular}

Table 6. List of all states which are not shared between the two phases.

the states in table 6 cancel out of the leading term in any elliptic genus refined by any of the displayed nonanomalous global symmetries, as before.

Finally, we compare the chiral states from the first GLSM, in tables 1 and 2, with those from the second GLSM, in tables 4 and 5. It is straightforward to check that they match - the states which are believed to flow to the IR in the first GLSM, are isomorphic to states in the second GLSM which are believed to flow to the IR. (States that mismatch between two phases of the GLSM, we do not consider, as we do not believe they flow to the IR.) This supports the triality proposal, in that it is a check that not only phases of a single GLSM, but phases of multiple GLSMs, all have the same IR limit.

In passing, we have seen how non-protected operators can pair up and become massive along RG flow, but in principle the opposite can also happen - pairs of massive operators can become massless and enter the RG flow. We do not seem to observe this in any of the examples discussed in this paper, and we leave open the question of whether more general triality examples exhibit that phenomenon, or whether for some reason it does not happen in UV presentation sof triality.

\subsection{Second example}

\subsubsection{First GLSM}

Next, we shall consider a (0,2) GLSM with $k=1, A=4$, and $n=2$, so $2 k+A-n=4$. Its two phases are defined by

$$
\mathcal{E} \equiv U \otimes S+V \otimes Q^{*}+W \otimes \operatorname{det} S^{*} \longrightarrow \mathbb{P}^{1}=\mathbb{P} \tilde{V}^{*}
$$

for $r \gg 0$, and

$$
\mathcal{F} \equiv U \otimes S^{*}+\tilde{V} \otimes Q^{*}+W \otimes \operatorname{det} S \longrightarrow \mathbb{P}^{3}=\mathbb{P} V^{*}
$$

for $r \ll 0$, where in both phases,

$$
U=\mathbb{C}^{4}, \quad V=\mathbb{C}^{4}, \quad W=\mathbb{C}^{2}, \quad \tilde{V}=\mathbb{C}^{2} .
$$


Each phase has a set of global nonanomalous $\mathrm{U}(1)^{3}$ symmetries, which are given by

\begin{tabular}{|l|cccc|}
\hline & $\tilde{V}$ & $U$ & $V$ & $W$ \\
\hline $\mathrm{U}(1)_{(1)}$ & 0 & 0 & -1 & -2 \\
$\mathrm{U}(1)_{(2)}$ & 1 & 0 & 0 & -1 \\
$\mathrm{U}(1)_{(3)}$ & 0 & 1 & 0 & +2 \\
\hline
\end{tabular}

In a slight variation from $[24,25]$, we have assigned the same charge to all elements of $W$, for simplicity in comparing states.

As in the previous example, the $\mathbb{P}^{1}$ of the $r \gg 0$ phase is identified with $\mathbb{P} \tilde{V}^{*}$ rather than $\mathbb{P} \tilde{V}$.

These nonlinear sigma models are neither $\mathrm{A} / 2$ nor $\mathrm{B} / 2$-twistable; the Fock vacuum line bundle is nontrivial. In the $r \gg 0$ phase, we take the Fock vacuum line bundle to be

$$
\mathcal{L}=\left(Q^{*}\right)^{-2} .
$$

In the $r \ll 0$ phase, we take the Fock vacuum line bundle to be

$$
\mathcal{L}=K_{(-3)} S^{*} \otimes K_{(-1,-1,-1)} Q^{*}
$$

This vacuum is somewhat more interesting, as it has no cohomology, hence there are no chiral states in $H^{\bullet}\left(\wedge^{0} \mathcal{F} \otimes \mathcal{L}\right)$. The theory still has a Fock vacuum, but it does not define a nontrivial element of BRST cohomology.

In our previous examples, many of the states matched 'on the nose,' in representations of not only anomaly-free symmetries but also anomalous symmetries. In this example, there are no states that match in representations of anomalous symmetries, only in anomaly-free symmetries.

Matching states are listed in tables 7 and 8. (Because of the sheer number of states, they could not all be listed in a single table, so they were broken into two sets related by Serre duality.) Displayed are states, the wedge power of the gauge bundle involved, the cohomology degree in which they appear, the representation of

$$
\mathrm{SL}(U) \times \mathrm{SL}(V) \times \mathrm{SL}(W) \times \mathrm{SL}(\tilde{V})=\mathrm{SU}(4) \times \mathrm{SU}(4) \times \mathrm{SU}(2) \times \mathrm{SU}(2)
$$

and charges under the nonanomalous global

$$
\mathrm{U}(1)_{(1)} \times \mathrm{U}(1)_{(2)} \times \mathrm{U}(1)_{(3)}
$$

symmetries, in the same format as for the previous example. The $\mathrm{U}(1)^{3}$ charges listed include the fractional contributions from the Fock vacua. For $r \gg 0$, we compute that the Fock vacuum has charge $(+4,+2,-4)$, whereas for $r \ll 0$, we compute that the Fock vacuum has charge $(0,-2,-4)$.

Furthermore, as before, each geometric phase has a few states not possessed by the other phase. These are listed in table 9 . 


\begin{tabular}{|c|c|c|c|}
\hline $\begin{array}{c}r \gg 0 \\
\text { State, } \wedge^{\bullet} \mathcal{E}, H^{\bullet}\left(\mathbb{P}^{1}\right)\end{array}$ & $\begin{array}{c}r \ll 0 \\
\text { State, } \wedge^{\bullet} \mathcal{F}, H^{\bullet}\left(\mathbb{P}^{3}\right)\end{array}$ & Rep' & $\mathrm{U}(1)^{3}$ \\
\hline $\operatorname{Sym}^{2} \tilde{V}^{*}, 0,0$ & $\operatorname{Sym}^{2} \tilde{V} \otimes\left(\wedge^{4} V\right)^{-1}, 2,2$ & $(\mathbf{1}, \mathbf{1}, \mathbf{1}, \mathbf{3})$ & $(+4,0,-4)$ \\
\hline$U \otimes\left(\wedge^{2} \tilde{V}\right)^{-1} \otimes \tilde{V}^{*}, 1,0$ & $U \otimes \tilde{V} \otimes\left(\wedge^{4} V\right)^{-1}, 2,1$ & $(4,1,1,2)$ & $(+4,-1,-3)$ \\
\hline$V \otimes \tilde{V}^{*}, 1,0$ & $\left(\wedge^{2} \tilde{V}\right) \otimes \tilde{V} \otimes\left(\wedge^{4} V\right)^{-1} \otimes V, 3,2$ & $(1,4,1,2)$ & $(+3,+1,-4)$ \\
\hline$W \otimes K_{(1,-2)} \tilde{V}, 1,0$ & $W \otimes \operatorname{Sym}^{3} \tilde{V} \otimes\left(\wedge^{4} V\right)^{-1}, 4,3$ & $(\mathbf{1}, \mathbf{1}, 2,4)$ & $(+2,0,-2)$ \\
\hline$\wedge^{2} U \otimes\left(\wedge^{2} \tilde{V}\right)^{-2}, 2,0$ & $\wedge^{2} U \otimes\left(\wedge^{4} V\right)^{-1}, 2,0$ & $(6,1,1,1)$ & $(+4,-2,-2)$ \\
\hline$U \otimes V \otimes\left(\wedge^{2} \tilde{V}\right)^{-1}, 2,0$ & $U \otimes \wedge^{2} \tilde{V} \otimes\left(\wedge^{4} V\right)^{-1} \otimes V, 3,1$ & $(4,4,1,1)$ & $(+3,0,-3)$ \\
\hline$U \otimes W \otimes \operatorname{Sym}^{2} \tilde{V}^{*}, 2,0$ & $U \otimes W \otimes \operatorname{Sym}^{2} \tilde{V} \otimes\left(\wedge^{4} V\right)^{-1}, 4,2$ & $(4,1,2,3)$ & $(+2,-1,-1)$ \\
\hline$\wedge^{2} V, 2,0$ & $\left(\wedge^{2} \tilde{V}\right)^{2} \otimes\left(\wedge^{4} V\right)^{-1} \otimes \wedge^{2} V, 4,2$ & $(\mathbf{1}, \mathbf{6}, \mathbf{1}, \mathbf{1})$ & $(+2,+2,-4)$ \\
\hline$V \otimes W \otimes K_{(1,-1)} \tilde{V}, 2,0$ & $W \otimes K_{(3,1)} \tilde{V} \otimes K_{(0,-1,-1,-1)} V, 5,3$ & $(1,4,2,3)$ & $(+1,+1,-2)$ \\
\hline$\wedge^{2} U \otimes W \otimes K_{(-1,-2)} \tilde{V}, 3,0$ & $\wedge^{2} U \otimes \tilde{V} \otimes W \otimes\left(\wedge^{4} V\right)^{-1}, 4,1$ & $(6,1,2,2)$ & $(+2,-2,0)$ \\
\hline$U \otimes V \otimes W \otimes \tilde{V}^{*}, 3,0$ & $U \otimes W \otimes K_{(2,1)} \tilde{V} \otimes K_{(0,-1,-1,-1)} V, 5,2$ & $(4,4,2,2)$ & $(+1,0,-1)$ \\
\hline$U \otimes \wedge^{2} W \otimes K_{(+1,-2)} \tilde{V}, 3,0$ & $U \otimes \wedge^{2} W \otimes \operatorname{Sym}^{3} \tilde{V} \otimes\left(\wedge^{4} V\right)^{-1}, 6,3$ & $(4,1,1,4)$ & $(0,-1,+1)$ \\
\hline$\wedge^{2} V \otimes W \otimes \tilde{V}, 3,0$ & $W \otimes K_{(3,2)} \tilde{V} \otimes K_{(0,0,-1,-1)} V, 6,3$ & $(1,6,2,2)$ & $(0,+2,-2)$ \\
\hline$V \otimes \wedge^{2} W \otimes K_{(2,-1)} \tilde{V}, 3,0$ & $\wedge^{4} U \otimes \operatorname{Sym}^{3} \tilde{V} \otimes V, 7,0$ & $(1,4,1,4)$ & $(-1,+1,0)$ \\
\hline$\wedge^{4} U \otimes K_{(-3,-3)} \tilde{V}, 4,1$ & $\wedge^{2} W \otimes K_{(-2,-2,-2,-2)} V, 2,3$ & $(\mathbf{1}, \mathbf{1}, \mathbf{1}, \mathbf{1})$ & $(+4,-4,0)$ \\
\hline$\wedge^{3} U \otimes V \otimes K_{(-2,-2)} \tilde{V}, 4,1$ & $\wedge^{3} U \otimes K_{(0,-1,-1,-1)} V, 3,0$ & $(\overline{4}, 4,1,1)$ & $(+3,-2,-1)$ \\
\hline$\wedge^{3} U \otimes W \otimes K_{(-2,-2)} \tilde{V}, 4,0$ & $\wedge^{3} U \otimes W \otimes\left(\wedge^{4} V\right)^{-1}, 4,0$ & $(\overline{4}, 1,2,1)$ & $(+2,-3,+1)$ \\
\hline$\wedge^{2} U \otimes \wedge^{2} V \otimes \wedge^{2} \tilde{V}^{*}, 4,1$ & $\wedge^{2} U \otimes \wedge^{2} \tilde{V} \otimes \wedge^{2} V^{*}, 4,1$ & $(6,6,1,1)$ & $(+2,0,-2)$ \\
\hline$\wedge^{2} U \otimes V \otimes W \otimes \wedge^{2} \tilde{V}^{*}, 4,0$ & $\wedge^{2} U \otimes W \otimes \wedge^{2} \tilde{V} \otimes \wedge^{3} V^{*}, 5,1$ & $(6,4,2,1)$ & $(+1,-1,0)$ \\
\hline$\wedge^{2} U \otimes \wedge^{2} W \otimes \operatorname{Sym}^{2} \tilde{V}^{*}, 4,0$ & $\wedge^{2} U \otimes \wedge^{2} W \otimes K_{(2,0)} \tilde{V} \otimes\left(\wedge^{4} V\right)^{-1}, 6,2$ & $(6,1,1,3)$ & $(0,-2,+2)$ \\
\hline$U \otimes \wedge^{3} V, 4,1$ & $U \otimes K_{(2,2)} \tilde{V} \otimes V^{*}, 5,2$ & $(4, \overline{4}, 1,1)$ & $(+1,+2,-3)$ \\
\hline$U \otimes \wedge^{2} V \otimes W, 4,0$ & $U \otimes W \otimes K_{(2,2)} \tilde{V} \otimes \wedge^{2} V^{*}, 6,2$ & $(4,6,2,1)$ & $(0,+1,-1)$ \\
\hline$U \otimes V \otimes \wedge^{2} W \otimes K_{(1,-1)} \tilde{V}, 4,0$ & $U \otimes \wedge^{2} W \otimes K_{(3,1)} \tilde{V} \otimes \wedge^{3} V^{*}, 7,3$ & $(4,4,1,3)$ & $(-1,0,+1)$ \\
\hline$\wedge^{4} V \otimes \wedge^{2} \tilde{V}, 4,1$ & $\left(\wedge^{2} \tilde{V}\right)^{3}, 6,3$ & $(\mathbf{1}, \mathbf{1}, \mathbf{1}, \mathbf{1})$ & $(0,+4,-4)$ \\
\hline$\wedge^{3} V \otimes W \otimes \wedge^{2} \tilde{V}, 4,0$ & $W \otimes K_{(3,3)} \tilde{V} \otimes V^{*}, 7,3$ & $(\mathbf{1}, \overline{4}, 2,1)$ & $(-1,+3,-2)$ \\
\hline$\wedge^{2} V \otimes \wedge^{2} W \otimes \operatorname{Sym}^{2} \tilde{V}, 4,0$ & $\wedge^{4} U \otimes K_{(3,1)} \tilde{V} \otimes \wedge^{2} V, 8,0$ & $(1,6,1,3)$ & $(-2,+2,0)$ \\
\hline$\wedge^{4} U \otimes V \otimes K_{(-2,-3)} \tilde{V}, 5,1$ & $\wedge^{2} W \otimes \tilde{V} \otimes K_{(-1,-2,-2,-2)} V, 3,3$ & $(\mathbf{1}, \mathbf{4}, \mathbf{1}, \mathbf{2})$ & $(+3,-3,0)$ \\
\hline$\wedge^{3} U \otimes \wedge^{2} V \otimes K_{(-1,-2)} \tilde{V}, 5,1$ & $\wedge^{3} U \otimes \tilde{V} \otimes \wedge^{2} V^{*}, 4,0$ & $(\overline{4}, 6,1,2)$ & $(+2,-1,-1)$ \\
\hline$\wedge^{3} U \otimes \wedge^{2} W \otimes K_{(-1,-2)} \tilde{V}, 5,0$ & $\wedge^{3} U \otimes \wedge^{2} W \otimes \tilde{V} \otimes \wedge^{4} V^{*}, 6,1$ & $(\overline{4}, 1,1,2)$ & $(0,-3,+3)$ \\
\hline$\wedge^{2} U \otimes \wedge^{3} V \otimes \tilde{V}^{*}, 5,1$ & $\wedge^{2} U \otimes K_{(2,1)} \tilde{V} \otimes V^{*}, 5,1$ & $(6, \overline{4}, 1,2)$ & $(+1,+1,-2)$ \\
\hline$\wedge^{2} U \otimes V \otimes \wedge^{2} W \otimes \tilde{V}^{*}, 5,0$ & $\wedge^{2} U \otimes \wedge^{2} W \otimes K_{(2,1)} \tilde{V} \otimes \wedge^{3} V^{*}, 7,2$ & $(6,4,1,2)$ & $(-1,-1,+2)$ \\
\hline$U \otimes \wedge^{4} V \otimes \tilde{V}, 5,1$ & $U \otimes K_{(3,2)} \tilde{V}, 6,2$ & $(4,1,1,2)$ & $(0,+3,-3)$ \\
\hline$U \otimes \wedge^{2} V \otimes \wedge^{2} W \otimes \tilde{V}, 5,0$ & $U \otimes \wedge^{2} W \otimes K_{(3,2)} \tilde{V} \otimes \wedge^{2} V^{*}, 8,3$ & $(4,6,1,2)$ & $(-2,+1,+1)$ \\
\hline$\wedge^{3} V \otimes \wedge^{2} W \otimes K_{(2,1)} \tilde{V}, 5,0$ & $\wedge^{4} U \otimes K_{(3,2)} \tilde{V} \otimes \wedge^{3} V, 9,0$ & $(1, \overline{4}, 1,2)$ & $(-3,+3,0)$ \\
\hline
\end{tabular}

Table 7. First half of shared states defined by matching representations of anomaly-free global symmetries.

As a consistency check, note that all of the states come in Serre dual pairs, just as in the previous example. In addition, the mismatched states come in pairs that cancel out of refined elliptic genus computations, as before, which means they can plausibly become massive along the RG flow. 


\begin{tabular}{|c|c|c|c|}
\hline $\begin{array}{c}r \gg 0 \\
\text { State, } \wedge^{\bullet} \mathcal{E}, H^{\bullet}\left(\mathbb{P}^{1}\right)\end{array}$ & $\begin{array}{c}r \ll 0 \\
\text { State, } \wedge^{\bullet} \mathcal{F}, H^{\bullet}\left(\mathbb{P}^{3}\right)\end{array}$ & Rep' & $\mathrm{U}(1)^{3}$ \\
\hline$\wedge^{4} U \otimes \wedge^{2} V \otimes K_{(-1,-3)} \tilde{V}, 6,1$ & $\wedge^{2} W \otimes \mathrm{S}^{2} \tilde{V} \otimes K_{(2,2,1,1)} V^{*}, 4,3$ & $(\mathbf{1}, \mathbf{6}, \mathbf{1}, \mathbf{3})$ & $(+2,-2,0)$ \\
\hline$\wedge^{4} U \otimes V \otimes W \otimes K_{(-2,-2)} \tilde{V}, 6,1$ & $\wedge^{4} U \otimes W \otimes \wedge^{3} V^{*}, 5,0$ & $(1,4,2,1)$ & $(+1,-3,+2)$ \\
\hline$\wedge^{4} U \otimes \wedge^{2} W \otimes K_{(-2,-2)} \tilde{V}, 6,0$ & $\wedge^{4} U \otimes \wedge^{2} W \otimes \wedge^{4} V^{*}, 6,0$ & $(\mathbf{1}, \mathbf{1}, \mathbf{1}, \mathbf{1})$ & $(0,-4,+4)$ \\
\hline$\wedge^{3} U \otimes \wedge^{3} V \otimes \operatorname{Sym}^{2} \tilde{V}^{*}, 6,1$ & $\wedge^{3} U \otimes \operatorname{Sym}^{2} \tilde{V} \otimes V^{*}, 5,0$ & $(\overline{4}, \overline{4}, \mathbf{1}, \mathbf{3})$ & $(+1,0,-1)$ \\
\hline$\wedge^{3} U \otimes \wedge^{2} V \otimes W \otimes \wedge^{2} \tilde{V}^{*}, 6,1$ & $\wedge^{3} U \otimes W \otimes \wedge^{2} \tilde{V} \otimes \wedge^{2} V^{*}, 6,1$ & $(\overline{4}, 6,2,1)$ & $(0,-1,+1)$ \\
\hline$\wedge^{3} U \otimes V \otimes \wedge^{2} W \otimes \wedge^{2} \tilde{V}^{*}, 6,0$ & $\wedge^{3} U \otimes \wedge^{2} W \otimes \wedge^{2} \tilde{V} \otimes \wedge^{3} V^{*}, 7,1$ & $(\overline{4}, 4,1,1)$ & $(-1,-2,+3)$ \\
\hline$\wedge^{2} U \otimes \wedge^{4} V \otimes K_{(1,-1)} \tilde{V}, 6,1$ & $\wedge^{2} U \otimes K_{(3,1)} \tilde{V}, 6,1$ & $(6,1,1,3)$ & $(0,+2,-2)$ \\
\hline$\wedge^{2} U \otimes \wedge^{3} V \otimes W, 6,1$ & $\wedge^{2} U \otimes W \otimes K_{(2,2)} \tilde{V} \otimes V^{*}, 7,2$ & $(6, \overline{4}, 2,1)$ & $(-1,+1,0)$ \\
\hline$\wedge^{2} U \otimes \wedge^{2} V \otimes \wedge^{2} W, 6,0$ & $\wedge^{2} U \otimes \wedge^{2} W \otimes\left(\wedge^{2} \tilde{V}\right)^{2} \otimes \wedge^{2} V^{*}, 8,2$ & $(6,6,1,1)$ & $(-2,0,+2)$ \\
\hline$U \otimes \wedge^{4} V \otimes W \otimes \wedge^{2} \tilde{V}, 6,1$ & $U \otimes W \otimes K_{(3,3)} \tilde{V}, 8,3$ & $(4,1,2,1)$ & $(-2,+3,-1)$ \\
\hline$U \otimes \wedge^{3} V \otimes \wedge^{2} W \otimes \wedge^{2} \tilde{V}, 6,0$ & $U \otimes K_{(3,3)} \tilde{V} \otimes \wedge^{2} W \otimes V^{*}, 9,3$ & $(4, \overline{4}, 1,1)$ & $(-3,+2,+1)$ \\
\hline$\wedge^{4} V \otimes \wedge^{2} W \otimes K_{(2,2)} \tilde{V}, 6,0$ & $\wedge^{4} U \otimes K_{(3,3)} \tilde{V} \otimes \wedge^{4} V, 10,0$ & $(\mathbf{1}, \mathbf{1}, \mathbf{1}, \mathbf{1})$ & $(-4,+4,0)$ \\
\hline$\wedge^{4} U \otimes \wedge^{3} V \otimes \operatorname{Sym}^{3} \tilde{V}^{*}, 7,1$ & $\wedge^{2} W \otimes S^{3} \tilde{V} \otimes K_{(2,1,1,1)} V^{*}, 5,3$ & $(\mathbf{1}, \overline{4}, \mathbf{1}, 4)$ & $(+1,-1,0)$ \\
\hline$\wedge^{4} U \otimes \wedge^{2} V \otimes W \otimes K_{(2,1)} \tilde{V}^{*}, 7,1$ & $\wedge^{4} U \otimes \tilde{V} \otimes W \otimes \wedge^{2} V^{*}, 6,0$ & $(1,6,2,2)$ & $(0,-2,+2)$ \\
\hline$\wedge^{3} U \otimes \wedge^{4} V \otimes K_{(1,-2)} \tilde{V}, 7,1$ & $\wedge^{3} U \otimes \operatorname{Sym}^{3} \tilde{V}, 6,0$ & $(\overline{4}, 1,1,4)$ & $(0,+1,-1)$ \\
\hline$\wedge^{3} U \otimes \wedge^{3} V \otimes W \otimes \tilde{V}^{*}, 7,1$ & $\wedge^{3} U \otimes W \otimes K_{(2,1)} \tilde{V} \otimes V^{*}, 7,1$ & $(\overline{4}, \overline{4}, 2,2)$ & $(-1,0,+1)$ \\
\hline$\wedge^{2} U \otimes \wedge^{4} V \otimes W \otimes \tilde{V}, 7,1$ & $\wedge^{2} U \otimes W \otimes K_{(3,2)} \tilde{V}, 8,2$ & $(6,1,2,2)$ & $(-2,+2,0)$ \\
\hline$\wedge^{4} U \otimes \wedge^{3} V \otimes W \otimes \operatorname{Sym}^{2} \tilde{V}^{*}, 8,1$ & $\wedge^{4} U \otimes W \otimes \operatorname{Sym}^{2} \tilde{V} \otimes V^{*}, 7,0$ & $(\mathbf{1}, \overline{4}, 2,3)$ & $(-1,-1,+2)$ \\
\hline$\wedge^{4} U \otimes \wedge^{2} V \otimes \wedge^{2} W \otimes \wedge^{2} \tilde{V}^{*}, 8,1$ & $\wedge^{4} U \otimes \wedge^{2} W \otimes \wedge^{2} \tilde{V} \otimes \wedge^{2} V^{*}, 8,1$ & $(\mathbf{1}, \mathbf{6}, \mathbf{1}, \mathbf{1})$ & $(-2,-2,+4)$ \\
\hline$\wedge^{3} U \otimes \wedge^{4} V \otimes W \otimes K_{(1,-1)} \tilde{V}, 8,1$ & $\wedge^{3} U \otimes W \otimes K_{(3,1)} \tilde{V}, 8,1$ & $(\overline{4}, 1,2,3)$ & $(-2,+1,+1)$ \\
\hline$\wedge^{3} U \otimes \wedge^{3} V \otimes \wedge^{2} W, 8,1$ & $\wedge^{3} U \otimes \wedge^{2} W \otimes K_{(2,2)} \tilde{V} \otimes V^{*}, 9,2$ & $(\overline{4}, \overline{4}, \mathbf{1}, \mathbf{1})$ & $(-3,0,+3)$ \\
\hline$\wedge^{2} U \otimes \wedge^{4} V \otimes \wedge^{2} W \otimes \wedge^{2} \tilde{V}, 8,1$ & $\wedge^{2} U \otimes K_{(3,3)} \tilde{V} \otimes \wedge^{2} W, 10,3$ & $(6,1,1,1)$ & $(-4,+2,+2)$ \\
\hline$\wedge^{4} U \otimes \wedge^{4} V \otimes W \otimes K_{(1,-2)} \tilde{V}, 9,1$ & $\wedge^{4} U \otimes \operatorname{Sym}^{3} \tilde{V} \otimes W, 8,0$ & $(1,1,2,4)$ & $(-2,0,+2)$ \\
\hline$\wedge^{4} U \otimes \wedge^{3} V \otimes \wedge^{2} W \otimes \tilde{V}^{*}, 9,1$ & $\wedge^{4} U \otimes \wedge^{2} W \otimes K_{(2,1)} \tilde{V} \otimes V^{*}, 9,1$ & $(\mathbf{1}, \overline{4}, 1,2)$ & $(-3,-1,+4)$ \\
\hline$\wedge^{3} U \otimes \wedge \wedge^{4} V \otimes \wedge^{2} W \otimes \tilde{V}, 9,1$ & $\wedge^{3} U \otimes \wedge^{2} W \otimes K_{(3,2)} \tilde{V}, 10,2$ & $(\overline{4}, 1,1,2)$ & $(-4,+1,+3)$ \\
\hline$\wedge^{4} U \otimes \wedge^{4} V \otimes \wedge^{2} W \otimes K_{(1,-1)} \tilde{V}, 10,1$ & $\wedge^{4} U \otimes \wedge^{2} W \otimes K_{(3,1)} \tilde{V}, 10,1$ & $(\mathbf{1}, \mathbf{1}, \mathbf{1}, \mathbf{3})$ & $(-4,0,+4)$ \\
\hline
\end{tabular}

Table 8. Second half of shared states defined by matching representations of anomaly-free global symmetries. We have occasionally used the symbol $\mathrm{S}$ as an abbreviation for Sym.

In the IR, these theories are believed to flow to an SCFT in which the nonanomalous global symmetry

$$
\mathrm{SU}(U) \times \mathrm{SU}(V) \times \mathrm{SU}(W) \times \mathrm{SU}(\tilde{V})
$$

is enhanced to an affine symmetry

$$
\mathrm{SU}(U)_{1} \times \mathrm{SU}(V)_{1} \times \mathrm{SU}(W)_{1} \times \mathrm{SU}(\tilde{V})_{3}=\mathrm{SU}(4)_{1} \times \mathrm{SU}(4)_{1} \times \mathrm{SU}(2)_{1} \times \mathrm{SU}(2)_{3},
$$

and the states in the IR should all be associated with integrable representations of the affine algebra above. Using the fact that the integrable representations of $\mathrm{SU}(2)_{3}$ are given by

$$
1, \quad 2,3,4,
$$

and the integrable representations of $\mathrm{SU}(4)_{1}$ are given by

$$
\mathbf{1}, \quad \mathbf{4}, \wedge^{2} \mathbf{4}=\mathbf{6}, \wedge^{3} \mathbf{4}=\overline{\mathbf{4}},
$$




\begin{tabular}{|c|c|c|c|c|c|c|}
\hline \multirow[b]{2}{*}{ State } & \multicolumn{2}{|c|}{$r \gg 0$} & \multicolumn{2}{|c|}{$r \ll 0$} & \multirow[b]{2}{*}{ Rep' } & \multirow[b]{2}{*}{$\mathrm{U}(1)^{3}$} \\
\hline & $\wedge \bullet \mathcal{E}$ & $H^{\bullet}\left(\mathbb{P}^{2}\right)$ & $\wedge \cdot \mathcal{F}$ & $H^{\bullet}\left(\mathbb{P}^{1}\right)$ & & \\
\hline$\wedge^{2} W \otimes K_{(2,-2)} \tilde{V}$ & 2 & 0 & - & - & $(1,1,1,5)$ & $(0,0,0)$ \\
\hline$\wedge^{4} U \otimes \wedge \wedge^{4} V \otimes K_{(1,-3)} \tilde{V}$ & 8 & 1 & - & - & $(\mathbf{1}, \mathbf{1}, \mathbf{1}, \mathbf{5})$ & $(0,0,0)$ \\
\hline$\wedge^{4} U \otimes K_{(+1,-1,-1,-1)} V$ & - & - & 4 & 0 & $(\mathbf{1}, \mathbf{1 0}, \mathbf{1}, \mathbf{1})$ & $(+2,-2,0)$ \\
\hline$\wedge^{2} W \otimes \wedge^{2} \tilde{V} \otimes K_{(0,-2,-2,-2)} V$ & - & - & 4 & 3 & $(\mathbf{1}, \overline{\mathbf{1 0}}, \mathbf{1}, \mathbf{1})$ & $(+2,-2,0)$ \\
\hline$\left.\wedge \wedge^{4} U \otimes \tilde{V} \otimes K_{(+1,0,-1,-1}\right) V$ & - & - & 5 & 0 & $(1,20,1,2)$ & $(+1,-1,0)$ \\
\hline$\wedge^{2} W \otimes K_{(2,1)} \tilde{V} \otimes K_{(0,-1,-2,-2)} V$ & - & - & 5 & 3 & $(1,20,1,2)$ & $(+1,-1,0)$ \\
\hline$\wedge^{4} U \otimes \wedge^{2} \tilde{V} \otimes K_{(+1,+1,-1,-1)} V$ & - & - & 6 & 0 & $(\mathbf{1}, \mathbf{2 0}, \mathbf{1}, \mathbf{1})$ & $(0,0,0)$ \\
\hline$\wedge^{4} U \otimes \operatorname{Sym}^{2} \tilde{V} \otimes K_{(+1,0,0,-1)} V$ & - & - & 6 & 0 & $(1,15,1,3)$ & $(0,0,0)$ \\
\hline$\wedge^{2} W \otimes K_{(3,1)} \tilde{V} \otimes K_{(0,-1,-1,-2)} V$ & - & - & 6 & 3 & $(1,15,1,3)$ & $(0,0,0)$ \\
\hline$\wedge^{2} W \otimes K_{(2,2)} \tilde{V} \otimes K_{(0,0,-2,-2)} V$ & - & - & 6 & 3 & $(\mathbf{1}, \mathbf{2 0}, \mathbf{1}, \mathbf{1})$ & $(0,0,0)$ \\
\hline$\wedge^{4} U \otimes K_{(2,1)} \tilde{V} \otimes K_{(1,1,0,-1)} V$ & - & - & 7 & 0 & $(1,20,1,2)$ & $(-1,+1,0)$ \\
\hline$\wedge^{2} W \otimes K_{(3,2)} \tilde{V} \otimes K_{(0,0,-1,-2)} V$ & - & - & 7 & 3 & $1,20,1,2)$ & $(-1,+1,0)$ \\
\hline$\wedge^{4} U \otimes K_{(2,2)} \tilde{V} \otimes K_{(1,1,1,-1)} V$ & - & - & 8 & 0 & $(\mathbf{1}, \mathbf{1 0}, \mathbf{1}, \mathbf{1})$ & $(-2,+2,0)$ \\
\hline$K_{(3,3)} \tilde{V} \otimes \wedge^{2} W \otimes \operatorname{Sym}^{2} V^{*}$ & - & - & 8 & 3 & $(\mathbf{1}, \overline{\mathbf{1 0}}, \mathbf{1}, \mathbf{1})$ & $(-2,+2,0)$ \\
\hline
\end{tabular}

Table 9. List of all states which are not shared between the two phases.

it is straightforward to check explicitly that all of the matching states listed in tables 7 and 8 are indeed associated with integrable representations, whereas by contrast all of the non-matching states in table 9 are associated with non-integrable representations. As before, it is our expectation that the non-matching states listed in table 9 do not survive to the IR.

\subsubsection{Other GLSMs}

Now, let us compute the chiral states in another GLSM related to the previous one by triality. We shall see, as before, that states in integrable representations match between phases, and also that those same states match between GLSMs.

Just as in section 3.4.2, we can obtain the other GLSMs related by triality by cyclically permuting

$$
U \longrightarrow V \longrightarrow \tilde{V}^{*} \longrightarrow U \longrightarrow \cdots
$$

(and changing the gauge group). The three large-radius phases correspond to the bundles and spaces given by

$$
\begin{aligned}
& (1): U \otimes S+V \otimes Q^{*}+W \otimes \operatorname{det} S^{*} \longrightarrow G\left(1, \tilde{V}^{*}\right) \\
& (2): V \otimes S+\tilde{V}^{*} \otimes Q^{*}+W \otimes \operatorname{det} S^{*} \longrightarrow G(1, U) \\
& (3): \tilde{V}^{*} \otimes S+U \otimes Q^{*}+W \otimes \operatorname{det} S^{*} \longrightarrow G(3, V),
\end{aligned}
$$


and the three $r \ll 0$ phases are described by

$$
\begin{aligned}
& (1): U \otimes S^{*}+\tilde{V} \otimes Q^{*}+W \otimes \operatorname{det} S \longrightarrow G\left(1, V^{*}\right), \\
& (2): V \otimes S^{*}+U^{*} \otimes Q^{*}+W \otimes \operatorname{det} S \longrightarrow G(1, \tilde{V}), \\
& (3): \tilde{V}^{*} \otimes S^{*}+V^{*} \otimes Q^{*}+W \otimes \operatorname{det} S \longrightarrow G\left(3, U^{*}\right) .
\end{aligned}
$$

(As a consistency check, the $\mathrm{U}(1)^{3}$ global symmetry is nonanomalous in each of the six phases above.)

Note that the $r \gg 0$ phase of the first GLSM and the $r \ll 0$ phase of the second GLSM are closely related: if we exchange

$$
U \leftrightarrow V^{*}, \quad W \leftrightarrow W^{*}, \quad \tilde{V} \leftrightarrow \tilde{V}^{*},
$$

then we can map one theory into the other. Similarly, the $r \ll 0$ phase of the first GLSM and the $r \gg 0$ phase of the second. Therefore, rather than compute a new set of chiral states from scratch, we can re-use our existing computations to derive the states for the second GLSM.

The $\mathrm{U}(1)^{3}$ action that matches that of the previous GLSM is given by

\begin{tabular}{|l|cccc|}
\hline & $\tilde{V}$ & $U$ & $V$ & $W$ \\
\hline $\mathrm{U}(1)_{(1)}$ & 0 & 0 & -1 & -2 \\
$\mathrm{U}(1)_{(2)}$ & -1 & 0 & 0 & +1 \\
$\mathrm{U}(1)_{(3)}$ & 0 & 1 & 0 & +2 \\
\hline
\end{tabular}

The vacuum charges in the $r \ll 0$ and $r \gg 0$ phases are given by, respectively, $(-4,+2,+4)$ and $(-4,-2,0)$.

Our results for the second GLSM are listed in tables 10, 11, 12, and 13. Tables 10, 11, and 12 list states that match between the $r \gg 0$ and $r \ll 0$ phases; table 13 lists the remainder. It is straightforward to check that all states come in Serre dual pairs, that all of the matching states lie in integrable representations, and that the mismatched states do not lie in integrable representations. In addition, as before, the mismatched states naturally come in pairs such that they can make no net contribution to the (leading term of the) elliptic genus, refined by any of the listed nonanomalous symmetries.

In addition, it is straightforward to check that all of the matching states in this GLSM, are in one-to-one correspondence with matching states in the previous GLSM related by triality - for any matching state in this GLSM, one can find a matching state in the previous GLSM in the same representation of nonanomalous symmetries.

\subsection{Third example: $T_{222}$}

\subsubsection{First GLSM}

This example is the case in which $k=1, A=2$, and $n=2$, so $2 k+A-n=2$. There is only one geometry appearing in this example, namely the space $G(1,2)=\mathbb{P}^{1}$, with bundle

$$
\mathcal{E}=S^{2} \oplus\left(Q^{*}\right)^{2} \oplus\left(\operatorname{det} S^{*}\right)^{2} .
$$




\begin{tabular}{|c|c|c|c|}
\hline $\begin{array}{c}r \ll 0 \\
\text { State, } \wedge^{\bullet} \mathcal{E}, H^{\bullet}\left(\mathbb{P}^{1}\right)\end{array}$ & $\begin{array}{c}r \gg 0 \\
\text { State, } \wedge \bullet \mathcal{F}, H^{\bullet}\left(\mathbb{P}^{3}\right)\end{array}$ & Rep' & $\mathrm{U}(1)^{3}$ \\
\hline $\operatorname{Sym}^{2} \tilde{V}, 0,0$ & $\mathrm{Sym}^{2} \tilde{V}^{*} \otimes \wedge^{4} U, 2,2$ & $(1,1,1,3)$ & $(-4,0,+4)$ \\
\hline$V^{*} \otimes \wedge^{2} \tilde{V} \otimes \tilde{V}, 1,0$ & $V^{*} \otimes \tilde{V}^{*} \otimes \wedge^{4} U, 2,1$ & $(\mathbf{1}, \overline{4}, 1,2)$ & $(-3,-1,+4)$ \\
\hline$U^{*} \otimes \tilde{V}, 1,0$ & $\wedge^{2} \tilde{V}^{*} \otimes \tilde{V}^{*} \otimes \wedge^{4} U \otimes U^{*}, 3,2$ & $(\overline{4}, 1,1,2)$ & $(-4,+1,+3)$ \\
\hline$W^{*} \otimes K_{(1,-2)} \tilde{V}^{*}, 1,0$ & $W^{*} \otimes \operatorname{Sym}^{3} \tilde{V}^{*} \otimes \wedge^{4} U, 4,3$ & $(\mathbf{1}, \mathbf{1}, \mathbf{2}, \mathbf{4})$ & $(-2,0,+2)$ \\
\hline$\wedge^{2} V^{*} \otimes\left(\wedge^{2} \tilde{V}\right)^{2}, 2,0$ & $\wedge^{2} V^{*} \otimes \wedge^{4} U, 2,0$ & $(\mathbf{1}, \mathbf{6}, \mathbf{1}, \mathbf{1})$ & $(-2,-2,+4)$ \\
\hline$V^{*} \otimes U^{*} \otimes \wedge^{2} \tilde{V}, 2,0$ & $V^{*} \otimes \wedge^{2} \tilde{V}^{*} \otimes \wedge^{4} U \otimes U^{*}, 3,1$ & $(\overline{4}, \overline{4}, 1,1)$ & $(-3,0,+3)$ \\
\hline$V^{*} \otimes W^{*} \otimes \operatorname{Sym}^{2} \tilde{V}, 2,0$ & $V^{*} \otimes W^{*} \otimes \operatorname{Sym}^{2} \tilde{V}^{*} \otimes \wedge^{4} U, 4,2$ & $(\mathbf{1}, \overline{4}, \mathbf{2}, \mathbf{3})$ & $(-1,-1,+2)$ \\
\hline$\wedge^{2} U^{*}, 2,0$ & $\left(\wedge^{2} \tilde{V}^{*}\right)^{2} \otimes \wedge^{4} U \otimes \wedge^{2} U^{*}, 4,2$ & $(6,1,1,1)$ & $(-4,+2,+2)$ \\
\hline$U^{*} \otimes W^{*} \otimes K_{(1,-1)} \tilde{V}^{*}, 2,0$ & $W^{*} \otimes K_{(3,1)} \tilde{V}^{*} \otimes \wedge^{3} U, 5,3$ & $(\overline{4}, 1,2,3)$ & $(-2,+1,+1)$ \\
\hline$\wedge^{2} V^{*} \otimes W^{*} \otimes K_{(2,1)} \tilde{V}, 3,0$ & $\wedge^{2} V^{*} \otimes \tilde{V}^{*} \otimes W^{*} \otimes \wedge^{4} U, 4,1$ & $(1,6,2,2)$ & $(0,-2,+2)$ \\
\hline$V^{*} \otimes U^{*} \otimes W^{*} \otimes \tilde{V}, 3,0$ & $V^{*} \otimes W^{*} \otimes K_{(2,1)} \tilde{V}^{*} \otimes \wedge^{3} U, 5,2$ & $(\overline{4}, \overline{4}, 2,2)$ & $(-1,0,+1)$ \\
\hline$V^{*} \otimes \wedge^{2} W^{*} \otimes K_{(1,-2)} \tilde{V}^{*}, 3,0$ & $V^{*} \otimes \wedge^{2} W^{*} \otimes \operatorname{Sym}^{3} \tilde{V}^{*} \otimes \wedge^{4} U, 6,3$ & $(\mathbf{1}, \overline{4}, \mathbf{1}, \mathbf{4})$ & $(+1,-1,0)$ \\
\hline$\wedge^{2} U^{*} \otimes W^{*} \otimes \tilde{V}^{*}, 3,0$ & $W^{*} \otimes K_{(3,2)} \tilde{V}^{*} \otimes \wedge^{2} U, 6,3$ & $(6,1,2,2)$ & $(-2,+2,0)$ \\
\hline$U^{*} \otimes \wedge^{2} W^{*} \otimes K_{(2,-1)} \tilde{V}^{*}, 3,0$ & $\wedge^{4} V^{*} \otimes \operatorname{Sym}^{3} \tilde{V}^{*} \otimes U^{*}, 7,0$ & $(\overline{4}, 1,1,4)$ & $(0,+1,-1)$ \\
\hline$\wedge^{4} V^{*} \otimes K_{(3,3)} \tilde{V}, 4,1$ & $\wedge^{2} W^{*} \otimes K_{(2,2,2,2)} U, 2,3$ & $(\mathbf{1}, \mathbf{1}, \mathbf{1}, \mathbf{1})$ & $(0,-4,+4)$ \\
\hline$\wedge^{3} V^{*} \otimes U^{*} \otimes K_{(2,2)} \tilde{V}, 4,1$ & $\wedge^{3} V^{*} \otimes \wedge^{3} U, 3,0$ & $(\overline{4}, 4,1,1)$ & $(-1,-2,+3)$ \\
\hline$\wedge^{3} V^{*} \otimes W^{*} \otimes K_{(2,2)} \tilde{V}, 4,0$ & $\wedge^{3} V^{*} \otimes W^{*} \otimes \wedge^{4} U, 4,0$ & $(1,4,2,1)$ & $(+1,-3,+2)$ \\
\hline$\wedge^{2} V^{*} \otimes \wedge^{2} U^{*} \otimes \wedge^{2} \tilde{V}, 4,1$ & $\wedge^{2} V^{*} \otimes \wedge^{2} \tilde{V}^{*} \otimes \wedge^{2} U, 4,1$ & $(6,6,1,1)$ & $(-2,0,+2)$ \\
\hline$\wedge^{2} V^{*} \otimes U^{*} \otimes W^{*} \otimes \wedge^{2} \tilde{V}, 4,0$ & $\wedge^{2} V^{*} \otimes W^{*} \otimes \wedge^{2} \tilde{V}^{*} \otimes \wedge^{3} U, 5,1$ & $(\overline{4}, 6,2,1)$ & $(0,-1,+1)$ \\
\hline$\wedge^{2} V^{*} \otimes \wedge^{2} W^{*} \otimes \operatorname{Sym}^{2} \tilde{V}, 4,0$ & $\wedge^{2} V^{*} \otimes \wedge^{2} W^{*} \otimes K_{(2,0)} \tilde{V}^{*} \otimes \wedge^{4} U, 6,2$ & $(1,6,1,3)$ & $(+2,-2,0)$ \\
\hline$V^{*} \otimes \wedge^{3} U^{*}, 4,1$ & $V^{*} \otimes K_{(2,2)} \tilde{\tilde{V}}^{*} \otimes U, 5,2$ & $(4, \overline{4}, 1,1)$ & $(-3,+2,+1)$ \\
\hline$V^{*} \otimes \wedge^{2} U^{*} \otimes W^{*}, 4,0$ & $V^{*} \otimes W^{*} \otimes K_{(2,2)} \tilde{V}^{*} \otimes \wedge^{2} U, 6,2$ & $(6, \overline{4}, 2,1)$ & $(-1,+1,0)$ \\
\hline$V^{*} \otimes U^{*} \otimes \wedge^{2} W^{*} \otimes K_{(1,-1)} \tilde{V}^{*}, 4,0$ & $V^{*} \otimes \wedge^{2} W^{*} \otimes K_{(3,1)} \tilde{V}^{*} \otimes \wedge^{3} U, 7,3$ & $(\overline{4}, \overline{4}, 1,3)$ & $(+1,0,-1)$ \\
\hline$\wedge^{4} U^{*} \otimes \wedge^{2} \tilde{V}^{*}, 4,1$ & $\left(\wedge^{2} \tilde{V}^{*}\right)^{3}, 6,3$ & $(\mathbf{1}, \mathbf{1}, \mathbf{1}, \mathbf{1})$ & $(-4,+4,0)$ \\
\hline$\wedge^{3} U^{*} \otimes W^{*} \otimes \wedge^{2} \tilde{V}^{*}, 4,0$ & $W^{*} \otimes K_{(3,3)} \tilde{V}^{*} \otimes U, 7,3$ & $(4,1,2,1)$ & $(-2,+3,-1)$ \\
\hline$\wedge^{2} U^{*} \otimes \wedge^{2} W^{*} \otimes \operatorname{Sym}^{2} \tilde{V}^{*}, 4,0$ & $\wedge^{4} V^{*} \otimes K_{(3,1)} \tilde{V}^{*} \otimes \wedge^{2} U^{*}, 8,0$ & $(6,1,1,3)$ & $(0,+2,-2)$ \\
\hline$\wedge^{4} V^{*} \otimes U^{*} \otimes K_{(3,2)} \tilde{V}, 5,1$ & $\wedge^{2} W^{*} \otimes \tilde{V}^{*} \otimes K_{(2,2,2,1)} U, 3,3$ & $(\overline{4}, 1,1,2)$ & $(0,-3,+3)$ \\
\hline$\wedge^{3} V^{*} \otimes \wedge^{2} U^{*} \otimes K_{(2,1)} \tilde{V}, 5,1$ & $\wedge^{3} V^{*} \otimes \tilde{V}^{*} \otimes \wedge^{2} U, 4,0$ & $(6,4,1,2)$ & $(-1,-1,+2)$ \\
\hline$\wedge^{3} V^{*} \otimes \wedge^{2} W^{*} \otimes K_{(2,1)} \tilde{V}, 5,0$ & $\wedge^{3} V^{*} \otimes \wedge^{2} W^{*} \otimes \tilde{V}^{*} \otimes \wedge^{4} U, 6,1$ & $(1,4,1,2)$ & $(+3,-3,0)$ \\
\hline$\wedge^{2} V^{*} \otimes \wedge^{3} U^{*} \otimes \tilde{V}, 5,1$ & $\wedge^{2} V^{*} \otimes K_{(2,1)} \tilde{V}^{*} \otimes U, 5,1$ & $(4,6,1,2)$ & $(-2,+1,+1)$ \\
\hline$\wedge^{2} V^{*} \otimes U^{*} \otimes \wedge^{2} W^{*} \otimes \tilde{V}, 5,0$ & $\wedge^{2} V^{*} \otimes \wedge^{2} W^{*} \otimes K_{(2,1)} \tilde{V}^{*} \otimes \wedge^{3} U, 7,2$ & $(\overline{4}, 6,1,2)$ & $(+2,-1,-1)$ \\
\hline$V^{*} \otimes \wedge^{4} U^{*} \otimes \tilde{V}^{*}, 5,1$ & $V^{*} \otimes K_{(3,2)} \tilde{V}^{*}, 6,2$ & $(\mathbf{1}, \overline{4}, 1,2)$ & $(-3,+3,0)$ \\
\hline$V^{*} \otimes \wedge^{2} U^{*} \otimes \wedge^{2} W^{*} \otimes \tilde{V}^{*}, 5,0$ & $V^{*} \otimes \wedge^{2} W^{*} \otimes K_{(3,2)} \tilde{V}^{*} \otimes \wedge^{2} U, 8,3$ & $(6, \overline{4}, 1,2)$ & $(+1,+1,-2)$ \\
\hline$\wedge^{3} U^{*} \otimes \wedge^{2} W^{*} \otimes K_{(2,1)} \tilde{V}^{*}, 5,0$ & $\wedge^{4} V^{*} \otimes K_{(3,2)} \tilde{V}^{*} \otimes \wedge^{3} U^{*}, 9,0$ & $(4,1,1,2)$ & $(0,+3,-3)$ \\
\hline
\end{tabular}

Table 10. First half of shared states defined by matching representations of anomaly-free global symmetries.

In the notations above, we will consider the GLSM which for $r \gg 0$ describes

$$
\mathcal{E}=U \otimes S+V \otimes Q^{*}+W \otimes \operatorname{det} S^{*} \longrightarrow G\left(1, \tilde{V}^{*}\right)
$$

for

$$
U=\mathbb{C}^{2}=V=W=\tilde{V}
$$




\begin{tabular}{|c|c|c|c|}
\hline $\begin{array}{c}r \ll 0 \\
\text { State, } \wedge^{\bullet} \mathcal{E}, H^{\bullet}\left(\mathbb{P}^{1}\right)\end{array}$ & $\begin{array}{c}r \gg 0 \\
\text { State, } \wedge^{\bullet} \mathcal{F}, H^{\bullet}\left(\mathbb{P}^{3}\right)\end{array}$ & Rep' & $\mathrm{U}(1)^{3}$ \\
\hline$\wedge^{4} V^{*} \otimes \wedge^{2} U^{*} \otimes K_{(3,1)} \tilde{V}, 6,1$ & $\wedge^{2} W^{*} \otimes S^{2} \tilde{V}^{*} \otimes K_{(2,2,1,1)} U, 4,3$ & $(6,1,1,3)$ & $(0,-2,+2)$ \\
\hline$\wedge^{4} V^{*} \otimes U^{*} \otimes W^{*} \otimes K_{(2,2)} \tilde{V}, 6,1$ & $\wedge^{4} V^{*} \otimes W^{*} \otimes \wedge^{3} U, 5,0$ & $(\overline{4}, 1,2,1)$ & $(+2,-3,+1)$ \\
\hline$\wedge^{4} V^{*} \otimes \wedge^{2} W^{*} \otimes K_{(2,2)} \tilde{V}, 6,0$ & $\wedge^{4} V^{*} \otimes \wedge^{2} W^{*} \otimes \wedge^{4} U, 6,0$ & $(1,1,1,1)$ & $(+4,-4,0)$ \\
\hline$\wedge^{3} V^{*} \otimes \wedge^{3} U^{*} \otimes \operatorname{Sym}^{2} \tilde{V}, 6,1$ & $\wedge^{3} V^{*} \otimes \operatorname{Sym}^{2} \tilde{V}^{*} \otimes U, 5,0$ & $(4,4,1,3)$ & $(-1,0,+1)$ \\
\hline$\wedge^{3} V^{*} \otimes \wedge^{2} U^{*} \otimes W^{*} \otimes \wedge^{2} \tilde{V}, 6,1$ & $\wedge^{3} V^{*} \otimes W^{*} \otimes \wedge^{2} \tilde{V}^{*} \otimes \wedge^{2} U, 6,1$ & $(6,4,2,1)$ & $(+1,-1,0)$ \\
\hline$\wedge^{3} V^{*} \otimes U^{*} \otimes \wedge^{2} W^{*} \otimes \wedge^{2} \tilde{V}, 6,0$ & $\wedge^{3} V^{*} \otimes \wedge^{2} W^{*} \otimes \wedge^{2} \tilde{V}^{*} \otimes \wedge^{3} U, 7,1$ & $(\overline{4}, 4,1,1)$ & $(+3,-2,-1)$ \\
\hline$\wedge^{2} V^{*} \otimes \wedge^{4} U^{*} \otimes K_{(1,-1)} \tilde{V}^{*}, 6,1$ & $\wedge^{2} V^{*} \otimes K_{(3,1)} \tilde{V}^{*}, 6,1$ & $(\mathbf{1}, \mathbf{6}, \mathbf{1}, \mathbf{3})$ & $(-2,+2,0)$ \\
\hline$\wedge^{2} V^{*} \otimes \wedge^{3} U^{*} \otimes W^{*}, 6,1$ & $\wedge^{2} V^{*} \otimes W^{*} \otimes K_{(2,2)} \tilde{V}^{*} \otimes U, 7,2$ & $(4,6,2,1)$ & $(0,+1,-1)$ \\
\hline$\wedge^{2} V^{*} \otimes \wedge^{2} U^{*} \otimes \wedge^{2} W^{*}, 6,0$ & $\left(\wedge^{2} V \otimes \wedge^{2} W \otimes\left(\wedge^{2} \tilde{V}\right)^{2}\right)^{*} \otimes \wedge^{2} U, 8,2$ & $(6,6,1,1)$ & $(+2,0,-2)$ \\
\hline$V^{*} \otimes \wedge^{4} U^{*} \otimes W^{*} \otimes \wedge^{2} \tilde{V}^{*}, 6,1$ & $V^{*} \otimes W^{*} \otimes K_{(3,3)} \tilde{V}^{*}, 8,3$ & $(\mathbf{1}, \overline{4}, \mathbf{2}, \mathbf{1})$ & $(-1,+3,-2)$ \\
\hline$V^{*} \otimes \wedge^{3} U^{*} \otimes \wedge^{2} W^{*} \otimes \wedge^{2} \tilde{V}^{*}, 6,0$ & $V^{*} \otimes K_{(3,3)} \tilde{V}^{*} \otimes \wedge^{2} W^{*} \otimes U, 9,3$ & $(4, \overline{4}, 1,1)$ & $(+1,+2,-3)$ \\
\hline$\wedge^{4} U^{*} \otimes \wedge^{2} W^{*} \otimes K_{(2,2)} \tilde{V}^{*}, 6,0$ & $\wedge^{4} V^{*} \otimes K_{(3,3)} \tilde{V}^{*} \otimes \wedge^{4} U^{*}, 10,0$ & $(\mathbf{1}, \mathbf{1}, \mathbf{1}, \mathbf{1})$ & $(0,+4,-4)$ \\
\hline
\end{tabular}

Table 11. Second portion of shared states defined by matching representations of anomaly-free global symmetries. We have occasionally used the symbol S as an abbreviation for Sym.

\begin{tabular}{|c|c|c|c|}
\hline $\begin{array}{c}r \ll 0 \\
\text { State, } \wedge^{\bullet} \mathcal{E}, H^{\bullet}\left(\mathbb{P}^{1}\right)\end{array}$ & $\begin{array}{c}r \gg 0 \\
\text { State, } \wedge^{\bullet} \mathcal{F}, H^{\bullet}\left(\mathbb{P}^{3}\right)\end{array}$ & Rep' & $\mathrm{U}(1)^{3}$ \\
\hline$\wedge^{4} V^{*} \otimes \wedge^{3} U^{*} \otimes S^{3} \tilde{V}, 7,1$ & $\wedge^{2} W^{*} \otimes \mathrm{S}^{3} \tilde{V}^{*} \otimes K_{(2,1,1,1)} U, 5,3$ & $(4,1,1,4)$ & $(0,-1,+1)$ \\
\hline$\wedge^{4} V^{*} \otimes \wedge^{2} U^{*} \otimes W^{*} \otimes K_{(2,1)} \tilde{V}, 7,1$ & $\wedge^{4} V^{*} \otimes \tilde{V}^{*} \otimes W^{*} \otimes \wedge^{2} U, 6,0$ & $(6,1,2,2)$ & $(+2,-2,0)$ \\
\hline$\wedge^{3} V^{*} \otimes \wedge^{4} U^{*} \otimes K_{(2,-1)} \tilde{V}, 7,1$ & $\wedge^{3} V^{*} \otimes \operatorname{Sym}^{3} \tilde{V}^{*}, 6,0$ & $(1,4,1,4)$ & $(-1,+1,0)$ \\
\hline$\wedge^{3} V^{*} \otimes \wedge^{3} U^{*} \otimes W^{*} \otimes \tilde{V}, 7,1$ & $\wedge^{3} V^{*} \otimes W^{*} \otimes K_{(2,1)} \tilde{V}^{*} \otimes U, 7,1$ & $(4,4,2,2)$ & $(+1,0,-1)$ \\
\hline$\wedge^{2} V^{*} \otimes \wedge^{4} U^{*} \otimes W^{*} \otimes \tilde{V}^{*}, 7,1$ & $\wedge^{2} V^{*} \otimes W^{*} \otimes K_{(3,2)} \tilde{V}^{*}, 8,2$ & $(1,6,2,2)$ & $(0,+2,-2)$ \\
\hline$\wedge^{4} V^{*} \otimes \wedge^{3} U^{*} \otimes W^{*} \otimes \operatorname{Sym}^{2} \tilde{V}, 8,1$ & $\wedge^{4} V^{*} \otimes W^{*} \otimes \operatorname{Sym}^{2} \tilde{V}^{*} \otimes U, 7,0$ & $(4,1,2,3)$ & $(+2,-1,-1)$ \\
\hline$\wedge^{4} V^{*} \otimes \wedge^{2} U^{*} \otimes \wedge^{2} W^{*} \otimes \wedge^{2} \tilde{V}, 8,1$ & $\wedge^{4} V^{*} \otimes \wedge^{2} W^{*} \otimes \wedge^{2} \tilde{V}^{*} \otimes \wedge^{2} U, 8,1$ & $(6,1,1,1)$ & $(+4,-2,-2)$ \\
\hline$\left(\wedge^{3} V \otimes \wedge^{4} U \otimes W \otimes K_{(1,-1)} \tilde{V}\right)^{*}, 8,1$ & $\wedge^{3} V^{*} \otimes W^{*} \otimes K_{(3,1)} \tilde{V}^{*}, 8,1$ & $(\mathbf{1}, 4,2,3)$ & $(+1,+1,-2)$ \\
\hline$\wedge^{3} V^{*} \otimes \wedge^{3} U^{*} \otimes \wedge^{2} W^{*}, 8,1$ & $\left(\wedge^{3} V \otimes \wedge^{2} W \otimes K_{(2,2)} \tilde{V}\right)^{*} \otimes U, 9,2$ & $(4,4,1,1)$ & $(+3,0,-3)$ \\
\hline$\wedge^{2} V^{*} \otimes \wedge^{4} U^{*} \otimes \wedge^{2} W^{*} \otimes \wedge^{2} \tilde{V}^{*}, 8,1$ & $\wedge^{2} V^{*} \otimes K_{(3,3)} \tilde{V}^{*} \otimes \wedge^{2} W^{*}, 10,3$ & $(\mathbf{1}, \mathbf{6}, \mathbf{1}, \mathbf{1})$ & $(+2,+2,-4)$ \\
\hline$\wedge^{4} V^{*} \otimes \wedge^{4} U^{*} \otimes W^{*} \otimes K_{(2,-1)} \tilde{V}, 9,1$ & $\wedge^{4} V^{*} \otimes \operatorname{Sym}^{3} \tilde{V}^{*} \otimes W^{*}, 8,0$ & $(1,1,2,4)$ & $(+2,0,-2)$ \\
\hline$\wedge^{4} V^{*} \otimes \wedge^{3} U^{*} \otimes \wedge^{2} W^{*} \otimes \tilde{V}, 9,1$ & $\left(\wedge^{4} V \otimes \wedge^{2} W \otimes K_{(2,1)} \tilde{V}\right)^{*} \otimes U, 9,1$ & $(4,1,1,2)$ & $(+4,-1,-3)$ \\
\hline$\wedge^{3} V^{*} \otimes \wedge^{4} U^{*} \otimes \wedge^{2} W^{*} \otimes \tilde{V}^{*}, 9,1$ & $\wedge^{3} V^{*} \otimes \wedge^{2} W^{*} \otimes K_{(3,2)} \tilde{V}^{*}, 10,2$ & $(\mathbf{1}, \mathbf{2}, \mathbf{1}, \mathbf{2})$ & $(+3,+1,-4)$ \\
\hline$\left(\wedge^{4} V \otimes \wedge^{4} U \otimes \wedge^{2} W \otimes K_{(1,-1)} \tilde{V}\right)^{*}, 10,1$ & $\wedge^{4} V^{*} \otimes \wedge^{2} W^{*} \otimes K_{(3,1)} \tilde{V}^{*}, 10,1$ & $(\mathbf{1}, \mathbf{1}, \mathbf{1}, \mathbf{3})$ & $(+4,0,-4)$ \\
\hline
\end{tabular}

Table 12. Third portion of shared states defined by matching representations of anomaly-free global symmetries. We have occasionally used the symbol S as an abbreviation for Sym.

and which for $r \ll 0$ is described by

$$
\mathcal{F}=U \otimes S^{*}+\tilde{V} \otimes Q^{*}+W \otimes \operatorname{det} S \longrightarrow G\left(1, V^{*}\right)
$$

Both of the phases above are B/2-twistable without further dualization; the Fock vacuum line bundle is trivial. As noted earlier, we need to make a choice of presentation as powers of $S^{*}$ and $Q^{*}$ - although the choice does not enter the final nonanomalous 


\begin{tabular}{|c|c|c|c|c|c|c|}
\hline \multirow[b]{2}{*}{ State } & \multicolumn{2}{|c|}{$r \ll 0$} & \multicolumn{2}{|c|}{$r \gg 0$} & \multirow[b]{2}{*}{ Rep' } & \multirow[b]{2}{*}{$\mathrm{U}(1)^{3}$} \\
\hline & $\wedge \cdot \mathcal{E}$ & $H^{\bullet}\left(\mathbb{P}^{2}\right)$ & $\wedge \cdot \mathcal{F}$ & $H^{\bullet}\left(\mathbb{P}^{1}\right)$ & & \\
\hline$\wedge^{2} W^{*} \otimes K_{(2,-2)} \tilde{V}^{*}$ & 2 & 0 & - & - & $(\mathbf{1}, \mathbf{1}, \mathbf{1}, \mathbf{5})$ & $(0,0,0)$ \\
\hline$\wedge^{4} V^{*} \otimes \wedge{ }^{4} U^{*} \otimes K_{(3,-1)} \tilde{V}$ & 8 & 1 & - & - & $(\mathbf{1}, \mathbf{1}, \mathbf{1}, \mathbf{5})$ & $(0,0,0)$ \\
\hline$\wedge^{4} V^{*} \otimes K_{(1,1,1,-1)} U$ & - & - & 4 & 0 & $(10,1,1,1)$ & $(0,-2,+2)$ \\
\hline$\wedge^{2} W^{*} \otimes \wedge^{2} \tilde{V}^{*} \otimes K_{(2,2,2,0)} U$ & - & - & 4 & 3 & $(10,1,1,1)$ & $(0,-2,+2)$ \\
\hline$\wedge^{4} V^{*} \otimes \tilde{V}^{*} \otimes K_{(1,1,0,-1)} U$ & - & - & 5 & 0 & $(20,1,1,2)$ & $(0,-1,+1)$ \\
\hline$\wedge^{2} W^{*} \otimes K_{(2,1)} \tilde{V}^{*} \otimes K_{(2,2,1,0)} U$ & - & - & 5 & 3 & $(20,1,1,2)$ & $(0,-1,+1)$ \\
\hline$\wedge^{4} V^{*} \otimes \wedge^{2} \tilde{V}^{*} \otimes K_{(1,1,-1,-1)} U$ & - & - & 6 & 0 & $(20,1,1,1)$ & $(0,0,0)$ \\
\hline$\wedge^{4} V^{*} \otimes \operatorname{Sym}^{2} \tilde{V}^{*} \otimes K_{(1,0,0,-1)} U$ & - & - & 6 & 0 & $(15,1,1,3)$ & $(0,0,0)$ \\
\hline$\wedge^{2} W^{*} \otimes K_{(3,1)} \tilde{V}^{*} \otimes K_{(2,1,1,0)} U$ & - & - & 6 & 3 & $(15,1,1,3)$ & $(0,0,0)$ \\
\hline$\wedge^{2} W^{*} \otimes K_{(2,2)} \tilde{V}^{*} \otimes K_{(2,2,0,0)} U$ & - & - & 6 & 3 & $(20,1,1,1)$ & $(0,0,0)$ \\
\hline$\wedge^{4} V^{*} \otimes K_{(2,1)} \tilde{V}^{*} \otimes K_{(1,1,0,-1)} U^{*}$ & - & - & 7 & 0 & $(20,1,1,2)$ & $(0,+1,-1)$ \\
\hline$\wedge^{2} W^{*} \otimes K_{(3,2)} \tilde{V}^{*} \otimes K_{(2,1,0,0)} U$ & - & - & 7 & 3 & $(20,1,1,2)$ & $(0,+1,-1)$ \\
\hline$\wedge^{4} V^{*} \otimes K_{(2,2)} \tilde{V}^{*} \otimes K_{(1,-1,-1,-1)} U$ & - & - & 8 & 0 & $(\mathbf{1 0}, \mathbf{1}, \mathbf{1}, \mathbf{1})$ & $(0,+2,-2)$ \\
\hline$K_{(3,3)} \tilde{V}^{*} \otimes \wedge^{2} W^{*} \otimes \operatorname{Sym}^{2} U$ & - & - & 8 & 3 & $(10,1,1,1)$ & $(0,+2,-2)$ \\
\hline
\end{tabular}

Table 13. List of all states which are not shared between the two phases.

representations, we must make a choice in order to initially compute the chiral states. We choose the canonical trivial presentation, as $K_{(0)} S^{*} \otimes K_{(0)} Q^{*}$ in both phases.

We will take the nonanomalous (chiral) global $\mathrm{U}(1)^{3}$ to be defined by

\begin{tabular}{|l|cccc|}
\hline & $\tilde{V}$ & $U$ & $V$ & $W$ \\
\hline $\mathrm{U}(1)_{(1)}$ & 0 & 0 & -1 & -1 \\
$\mathrm{U}(1)_{(2)}$ & 1 & 0 & 0 & -1 \\
$\mathrm{U}(1)_{(3)}$ & 0 & 1 & 0 & 1 \\
\hline
\end{tabular}

For both the $r \gg 0$ and $r \ll 0$ phases, we compute that the Fock vacuum has charge $(+2,0,-2)$.

Table 14 lists all the states which match between the two phases of this GLSM. A few states do not match; these are listed in table 15. As a consistency check, note that both tables are invariant under Serre duality as expected.

It was predicted in [25, eq. (3.1)] that the $\mathrm{SU}(2)^{4}$ global flavor symmetries of this model should be promoted to an

$$
\mathrm{SU}(2)_{1} \times \mathrm{SU}(2)_{1} \times \mathrm{SU}(2)_{1} \times \mathrm{SU}(2)_{1}
$$

affine symmetry in the IR SCFT, and indeed, note that all of the matching representations in table 14 are integrable, whereas all of the mismatched representations in table 15 are non-integrable, suggesting that the mismatched representations do not survive to the IR. In addition, it is also easy to check that the mismatched states in table 15 cancel out of 


\begin{tabular}{|c|c|c|c|}
\hline $\begin{array}{c}r \gg 0 \\
\text { State, } \wedge^{\bullet} \mathcal{E}, H^{\bullet}\left(\mathbb{P}^{1}\right)\end{array}$ & $\begin{array}{c}r \ll 0 \\
\text { State, } \wedge^{\bullet} \mathcal{F}, H^{\bullet}\left(\mathbb{P}^{1}\right)\end{array}$ & Rep' & $\mathrm{U}(1)^{3}$ \\
\hline $1,0,0$ & $1,0,0$ & $(\mathbf{1}, \mathbf{1}, \mathbf{1}, \mathbf{1})$ & $(+2,0,-2)$ \\
\hline$W \otimes \tilde{V}, 1,0$ & $W \otimes \tilde{V}, 2,1$ & $(1,1,2,2)$ & $(+1,0,-1)$ \\
\hline$\wedge^{2} U \otimes K_{(-1,-1)} \tilde{V}, 2,1$ & $\wedge^{2} W \otimes K_{(-1,-1)} V, 2,1$ & $(\mathbf{1}, \mathbf{1}, \mathbf{1}, \mathbf{1})$ & $(+2,-2,0)$ \\
\hline$U \otimes V, 2,1$ & $U \otimes V, 1,0$ & $(2,2,1,1)$ & $(+1,0,-1)$ \\
\hline$U \otimes W, 2,0$ & $U \otimes W, 2,0$ & $(2,1,2,1)$ & $(+1,-1,0)$ \\
\hline$\wedge^{2} V \otimes \wedge^{2} \tilde{V}, 2,1$ & $\wedge^{2} V \otimes \wedge^{2} \tilde{V}, 2,1$ & $(\mathbf{1}, \mathbf{1}, \mathbf{1}, \mathbf{1})$ & $(0,+2,-2)$ \\
\hline$V \otimes W \otimes \wedge^{2} \tilde{V}, 2,0$ & $V \otimes W \otimes \wedge^{2} \tilde{V}, 3,1$ & $(1,2,2,1)$ & $(0,+1,-1)$ \\
\hline$\wedge^{2} U \otimes V \otimes \tilde{V}^{*}, 3,1$ & $\tilde{V} \otimes \wedge^{2} W \otimes V^{*}, 3,1$ & $(1,2,1,2)$ & $(+1,-1,0)$ \\
\hline$U \otimes \wedge^{2} V \otimes \tilde{V}, 3,1$ & $U \otimes \tilde{V} \otimes \wedge^{2} V, 2,0$ & $(2,1,1,2)$ & $(0,+1,-1)$ \\
\hline$U \otimes \wedge^{2} W \otimes \tilde{V}, 3,0$ & $U \otimes \wedge^{2} W \otimes \tilde{V}, 4,1$ & $(2,1,1,2)$ & $(0,-1,+1)$ \\
\hline$V \otimes \wedge^{2} W \otimes K_{(2,1)} \tilde{V}, 3,0$ & $\wedge^{2} U \otimes \tilde{V} \otimes K_{(2,1)} V, 3,0$ & $(1,2,1,2)$ & $(-1,+1,0)$ \\
\hline$\wedge^{2} U \otimes V \otimes W, 4,1$ & $\wedge^{2} U \otimes V \otimes W, 3,0$ & $(\mathbf{1}, \mathbf{2}, \mathbf{2}, \mathbf{1})$ & $(0,-1,+1)$ \\
\hline$\wedge^{2} U \otimes \wedge^{2} W, 4,0$ & $\wedge^{2} U \otimes \wedge^{2} W, 4,0$ & $(\mathbf{1}, \mathbf{1}, \mathbf{1}, \mathbf{1})$ & $(0,-2,+2)$ \\
\hline$U \otimes \wedge^{2} V \otimes W \otimes \wedge^{2} \tilde{V}, 4,1$ & $U \otimes \wedge^{2} V \otimes W \otimes \wedge^{2} \tilde{V}, 4,1$ & $(2,1,2,1)$ & $(-1,+1,0)$ \\
\hline$U \otimes V \otimes \wedge^{2} W \otimes \wedge^{2} \tilde{V}, 4,0$ & $U \otimes V \otimes \wedge^{2} W \otimes \wedge^{2} \tilde{V}, 5,1$ & $(2,2,1,1)$ & $(-1,0,+1)$ \\
\hline$\wedge^{2} V \otimes \wedge^{2} W \otimes K_{(2,2)} \tilde{V}, 4,0$ & $\wedge^{2} U \otimes \wedge^{2} \tilde{V} \otimes K_{(2,2)} V, 4,0$ & $(\mathbf{1}, \mathbf{1}, \mathbf{1}, \mathbf{1})$ & $(-2,+2,0)$ \\
\hline$\wedge^{2} U \otimes \wedge^{2} V \otimes W \otimes \tilde{V}, 5,1$ & $\wedge^{2} U \otimes \tilde{V} \otimes W \otimes \wedge^{2} V, 4,0$ & $(1,1,2,2)$ & $(-1,0,+1)$ \\
\hline$\wedge^{2} U \otimes \wedge^{2} V \otimes \wedge^{2} W \otimes \wedge^{2} \tilde{V}, 6,1$ & $\wedge^{2} U \otimes \wedge^{2} V \otimes \wedge^{2} W \otimes \wedge^{2} \tilde{V}, 6,1$ & $(\mathbf{1}, \mathbf{1}, \mathbf{1}, \mathbf{1})$ & $(-2,0,+2)$ \\
\hline
\end{tabular}

Table 14. Shared states defined by matching representations of anomaly-free global symmetries.

\begin{tabular}{|c|c|c|c|c|c|c|}
\hline \multirow[b]{2}{*}{ State } & \multicolumn{2}{|c|}{$r \gg 0$} & \multicolumn{2}{|c|}{$r \ll 0$} & \multirow[b]{2}{*}{ Rep' } & \multirow[b]{2}{*}{$\mathrm{U}(1)^{3}$} \\
\hline & $\wedge^{\bullet} \mathcal{E}$ & $H^{\bullet}\left(\mathbb{P}^{1}\right)$ & $\wedge \bullet \mathcal{F}$ & $H^{\bullet}\left(\mathbb{P}^{1}\right)$ & & \\
\hline$\wedge^{2} W \otimes K_{(2,0)} \tilde{V}$ & 2 & 0 & - & - & $(\mathbf{1}, \mathbf{1}, \mathbf{1}, \mathbf{3})$ & $(0,0,0)$ \\
\hline$\wedge^{2} U \otimes \wedge^{2} V \otimes K_{(1,-1)} \tilde{V}$ & 4 & 1 & - & - & $(\mathbf{1}, \mathbf{1}, \mathbf{1}, \mathbf{3})$ & $(0,0,0)$ \\
\hline$\wedge^{2} U \otimes K_{(2,0)} V$ & - & - & 2 & 0 & $(\mathbf{1}, \mathbf{3}, \mathbf{1}, \mathbf{1})$ & $(0,0,0)$ \\
\hline$\wedge^{2} \tilde{V} \otimes \wedge^{2} W \otimes K_{(1,-1)} V$ & - & - & 4 & 1 & $(\mathbf{1}, \mathbf{3}, \mathbf{1}, \mathbf{1})$ & $(0,0,0)$ \\
\hline
\end{tabular}

Table 15. List of all states which are not shared between the two phases.

the leading term in elliptic genera, refined by any listed nonanomalous symmetry, which is consistent with the prediction that they do not survive to the IR.

Another prediction of [25] is that in this particular model, the $\mathrm{SU}(2)_{1}^{4}$ affine symmetry of the IR SCFT should be enhanced to an $E_{6}$ symmetry at level 1 . Briefly, the $\mathrm{SU}(W)$ of the two fermi fields can combine with the $\mathrm{SU}(U)$ to form $\mathrm{SU}(4)$, and by triality, any one of the other $\mathrm{SU}(2)$ 's at a time. The resulting structure can be naturally interpreted as a subgroup of $E_{6}$.

We can see some evidence for this in the states above. In particular, the total number of matching states is 54, twice the dimension of the $\mathbf{2 7}$ representation, suggesting that the states above flow to copies of either the $\mathbf{2 7}$ or $\overline{\mathbf{2 7}}$ representations. Indeed, the only integrable representations of $E_{6}$ at level 1 are $\mathbf{1}, \mathbf{2 7}$, and $\overline{\mathbf{2 7}}$. 
Now that we have verified the dimensions are consistent, let us see if the precise representations appearing above match those in the decomposition of a $\mathbf{2 7}$ or $\overline{\mathbf{2 7}}$ under an $s u(2)^{4}$ subalgebra. ${ }^{10}$ We can think of the $s u(2)^{4}$ subalgebra of $E_{6}$ as obtained from a maximal $(\mathrm{SU}(2) \times \mathrm{SU}(6)) / \mathbb{Z}_{2}$ subgroup, and the $\mathrm{SU}(6)$ naturally contains an $\mathrm{SU}(2)^{3}$ from omitting two of the nodes in its Dynkin diagram. The $\mathrm{SU}(W)$, which can recombine with any one other $\mathrm{SU}(2)$, can be understood as the middle node in the $E_{6}$ Dynkin diagram, or equivalently the middle node in the $\mathrm{SU}(6)$ Dynkin diagram. Under the $(\mathrm{SU}(2) \times \mathrm{SU}(6)) / \mathbb{Z}_{2}$ subgroup, the $\mathbf{2 7}$ of $E_{6}$ decomposes as [58, table 15]

$$
\mathbf{2 7}=(\mathbf{2}, \overline{\mathbf{6}})+(\mathbf{1}, \mathbf{1 5}) .
$$

Furthermore, under the $\mathrm{SU}(2)^{3}$ subgroup of $\mathrm{SU}(6)$,

$$
\overline{\mathbf{6}}=(\mathbf{2}, \mathbf{1}, \mathbf{1})+(\mathbf{1}, \mathbf{2}, \mathbf{1})+(\mathbf{1}, \mathbf{1}, \mathbf{2}) .
$$

(The 6 has the same decomposition - the $s u(2)^{4}$ subalgebra cannot distinguish the 6 from $\overline{\mathbf{6}}$.) Similarly, the $\mathbf{1 5}=\wedge^{2} \mathbf{6}$ should decompose as

$$
\begin{aligned}
\mathbf{1 5} & =\wedge^{2}(\mathbf{2}, \mathbf{1}, \mathbf{1})+\wedge^{2}(\mathbf{1}, \mathbf{2}, \mathbf{1})+\wedge^{2}(\mathbf{1}, \mathbf{1}, \mathbf{2})+(\mathbf{2}, \mathbf{2}, \mathbf{1})+(\mathbf{2}, \mathbf{1}, \mathbf{2})+(\mathbf{1}, \mathbf{2}, \mathbf{2}), \\
& =3(\mathbf{1}, \mathbf{1}, \mathbf{1})+(\mathbf{2}, \mathbf{2}, \mathbf{1})+(\mathbf{2}, \mathbf{1}, \mathbf{2})+(\mathbf{1}, \mathbf{2}, \mathbf{2}) .
\end{aligned}
$$

Thus, under the $\left(\mathrm{SU}(2)^{4}\right) / \mathbf{Z}_{2}$ subgroup of $E_{6}$,

$$
\begin{aligned}
\mathbf{2 7}= & (\mathbf{2}, \mathbf{2}, \mathbf{1}, \mathbf{1})+(\mathbf{2}, \mathbf{1}, \mathbf{2}, \mathbf{1})+(\mathbf{2}, \mathbf{1}, \mathbf{1}, \mathbf{2})+(\mathbf{1}, \mathbf{2}, \mathbf{2}, \mathbf{1})+(\mathbf{1}, \mathbf{2}, \mathbf{1}, \mathbf{2})+(\mathbf{1}, \mathbf{1}, \mathbf{2}, \mathbf{2}) \\
& +3(\mathbf{1}, \mathbf{1}, \mathbf{1}, \mathbf{1}) .
\end{aligned}
$$

(The $\overline{\mathbf{2 7}}$ has the same decomposition — the $s u(2)^{4}$ subalgebra cannot distinguish $\mathbf{2 7}$ from $\overline{\mathbf{2 7}}$.) It is straightforward to check that the states in table 14 do indeed form two copies of the decomposition above, partially verifying that in the IR, the states transform (two) 27's or $\overline{\mathbf{2 7}}$ 's.

It may well be possible to say more. For example, [25, eq. (3.28)] contains a prediction for the left NS partition function, from which one could extract a counting of left NS states. However, our chiral ring computations above are in the left $\mathrm{R}$ sector, and for reasons discussed earlier, it is not entirely clear that those two sectors should match in these examples, so we will not pursue that direction further.

\subsubsection{Other GLSMs}

Other GLSMs related by triality can be constructed by cyclically exchanging the vector spaces

$$
U \mapsto V \mapsto \tilde{V}^{*} \mapsto U \mapsto \cdots
$$

It is straightforward to check that the same $\mathrm{U}(1)^{3}$ is nonanomalous for each GLSM so obtained; however, to match charges, we need to pick a slightly different $\mathrm{U}(1)^{3}$ action for

\footnotetext{
${ }^{10}$ We would like to thank A. Knutson for a discussion of the corresponding group theory.
} 
the different GLSMs. Moreover, since the dimensions of these vector spaces all match, we can immediately derive the chiral states from tables 14 and 15 for the first GLSM for $T_{2,2,2}$.

For completeness, we list in tables 16 and 17 the corresponding chiral states for one cyclic rotation, i.e. $U$ replaced with $V$ and so forth. The global $\mathrm{U}(1)^{3}$ charges listed are those for the action defined by

\begin{tabular}{|l|cccc|}
\hline & $\tilde{V}$ & $U$ & $V$ & $W$ \\
\hline $\mathrm{U}(1)_{(1)}$ & 0 & 0 & -1 & -1 \\
$\mathrm{U}(1)_{(2)}$ & -1 & 0 & 0 & +1 \\
$\mathrm{U}(1)_{(3)}$ & 0 & 1 & 0 & 1 \\
\hline
\end{tabular}

For both geometric phases, we compute that the Fock vacuum has charge $(+2,-2,0)$. As before, all states come in Serre-dual pairs which dualize representations. Also as before, all the representations appearing amongst matched states are integrable, whereas the unmatched states in table 17 have nonintegrable representations. Furthermore, the unmatched states cancel out of leading terms in refined elliptic genera, because they come in pairs with opposite chirality and matching representations of global symmetries.

With the $\mathrm{U}(1)^{3}$ charges above, it is straightforwrad to check that all of the states below in table 16, which match between the two phases of the second GLSM and are expected to survive to the IR, also appear in table 14, which listed the states of the first GLSM that are expected to survive to the IR. Thus, these rings provide evidence not only that different phases of each GLSM flow to the same IR fixed point, but that in addition, phases of related distinct GLSMs also flow to the same IR fixed point.

\section{Conclusions}

In this paper we have used chiral state computations to confirm the triality conjecture of $[24,25]$, which says that triples of two-dimensional $(0,2)$ theories RG flow to the same IR fixed point.

We began with a review of chiral states and rings in $(2,2)$ and $(0,2)$ theories. The fact that the Fock vacuum in a nonlinear sigma model transforms as a section of a line bundle over the target played an important role in our computations, and we elaborated on this property, and also explained how even in $(2,2)$ theories, this arises in describing choices of spin structures on the target space.

We then turned to chiral states in the theories arising in triality. As $(0,2)$ chiral states are not protected against nonperturbative corrections, and the theories in question RG flow from weakly-coupled nonlinear sigma models to strongly coupled regimes, the chiral states need not match identically everywhere along RG flow. (That said, the opposite has often been implicitly assumed, so triality provides clean examples demonstrating that $(0,2)$ chiral rings are not protected.) In several examples, we computed chiral states in various phases and different GLSMs related by triality, and indeed discovered that the states did not all match. However, the states that did match, were all consistent with the proposed affine symmetry algebras of the IR fixed point. Furthermore, the mismatched 


\begin{tabular}{|c|c|c|c|}
\hline $\begin{array}{c}r \gg 0 \\
\text { State, } \wedge^{\bullet} \mathcal{E}, H^{\bullet}\left(\mathbb{P}^{1}\right)\end{array}$ & $\begin{array}{c}r \ll 0 \\
\text { State, } \wedge^{\bullet} \mathcal{F}, H^{\bullet}\left(\mathbb{P}^{1}\right)\end{array}$ & Rep' & $\mathrm{U}(1)^{3}$ \\
\hline $1,0,0$ & $1,0,0$ & $(\mathbf{1}, \mathbf{1}, \mathbf{1}, \mathbf{1})$ & $(+2,-2,0)$ \\
\hline$W \otimes U^{*}, 1,0$ & $W \otimes U^{*}, 2,1$ & $(2,1,2,1)$ & $(+1,-1,0)$ \\
\hline$\wedge^{2} V \otimes K_{(-1,-1)} U^{*}, 2,1$ & $\wedge^{2} W \otimes K_{(-1,-1)} \tilde{V}^{*}, 2,1$ & $(\mathbf{1}, \mathbf{1}, \mathbf{1}, \mathbf{1})$ & $(0,-2,+2)$ \\
\hline$V \otimes \tilde{V}^{*}, 2,1$ & $V \otimes \tilde{V}^{*}, 1,0$ & $(1,2,1,2)$ & $(+1,-1,0)$ \\
\hline$V \otimes W, 2,0$ & $V \otimes W, 2,0$ & $(\mathbf{1}, 2,2,1)$ & $(0,-1,+1)$ \\
\hline$\wedge^{2} \tilde{V}^{*} \otimes \wedge^{2} U^{*}, 2,1$ & $\wedge^{2} \tilde{V}^{*} \otimes \wedge^{2} U^{*}, 2,1$ & $(\mathbf{1}, \mathbf{1}, \mathbf{1}, \mathbf{1})$ & $(+2,0,-2)$ \\
\hline$\tilde{V}^{*} \otimes W \otimes \wedge^{2} U^{*}, 2,0$ & $\tilde{V}^{*} \otimes W \otimes \wedge^{2} U^{*}, 3,1$ & $(1,1,2,2)$ & $(+1,0,-1)$ \\
\hline$\wedge^{2} V \otimes \tilde{V}^{*} \otimes U, 3,1$ & $U^{*} \otimes \wedge^{2} W \otimes \tilde{V}, 3,1$ & $(2,1,1,2)$ & $(0,-1,+1)$ \\
\hline$V \otimes \wedge^{2} \tilde{V}^{*} \otimes U^{*}, 3,1$ & $V \otimes U^{*} \otimes \wedge^{2} \tilde{V}^{*}, 2,0$ & $(\mathbf{2}, \mathbf{2}, \mathbf{1}, \mathbf{1})$ & $(+1,0,-1)$ \\
\hline$V \otimes \wedge^{2} W \otimes U^{*}, 3,0$ & $V \otimes \wedge^{2} W \otimes U^{*}, 4,1$ & $(2,2,1,1)$ & $(-1,0,+1)$ \\
\hline$\tilde{V}^{*} \otimes \wedge^{2} W \otimes K_{(2,1)} U^{*}, 3,0$ & $\wedge^{2} V \otimes U^{*} \otimes K_{(2,1)} \tilde{V}^{*}, 3,0$ & $(2,1,1,2)$ & $(0,+1,-1)$ \\
\hline$\wedge^{2} V \otimes \tilde{V}^{*} \otimes W, 4,1$ & $\wedge^{2} V \otimes \tilde{V}^{*} \otimes W, 3,0$ & $(\mathbf{1}, \mathbf{1}, \mathbf{2}, \mathbf{2})$ & $(-1,0,+1)$ \\
\hline$\wedge^{2} V \otimes \wedge^{2} W, 4,0$ & $\wedge^{2} V \otimes \wedge^{2} W, 4,0$ & $(\mathbf{1}, \mathbf{1}, \mathbf{1}, \mathbf{1})$ & $(-2,0,+2)$ \\
\hline$V \otimes \wedge^{2} \tilde{V}^{*} \otimes W \otimes \wedge^{2} U^{*}, 4,1$ & $V \otimes \wedge^{2} \tilde{V}^{*} \otimes W \otimes \wedge^{2} U^{*}, 4,1$ & $(1,2,2,1)$ & $(0,+1,-1)$ \\
\hline$V \otimes \tilde{V}^{*} \otimes \wedge^{2} W \otimes \wedge^{2} U^{*}, 4,0$ & $V \otimes \tilde{V}^{*} \otimes \wedge^{2} W \otimes \wedge^{2} U^{*}, 5,1$ & $(\mathbf{1}, \mathbf{2}, \mathbf{1}, \mathbf{2})$ & $(-1,+1,0)$ \\
\hline$\wedge^{2} \tilde{V}^{*} \otimes \wedge^{2} W \otimes K_{(2,2)} U^{*}, 4,0$ & $\wedge^{2} V \otimes \wedge^{2} U^{*} \otimes K_{(2,2)} \tilde{V}^{*}, 4,0$ & $(\mathbf{1}, \mathbf{1}, \mathbf{1}, \mathbf{1})$ & $(0,+2,-2)$ \\
\hline$\wedge^{2} V \otimes \wedge^{2} \tilde{V}^{*} \otimes W \otimes U^{*}, 5,1$ & $\wedge^{2} V \otimes U^{*} \otimes W \otimes \wedge^{2} \tilde{V}^{*}, 4,0$ & $(2,1,2,1)$ & $(-1,+1,0)$ \\
\hline$\wedge^{2} V \otimes \wedge^{2} \tilde{V}^{*} \otimes \wedge^{2} W \otimes \wedge^{2} U^{*}, 6,1$ & $\wedge^{2} V \otimes \wedge^{2} \tilde{V}^{*} \otimes \wedge^{2} W \otimes \wedge^{2} U^{*}, 6,1$ & $(\mathbf{1}, \mathbf{1}, \mathbf{1}, \mathbf{1})$ & $(-2,+2,0)$ \\
\hline
\end{tabular}

Table 16. Shared states defined by matching representations of anomaly-free global symmetries.

\begin{tabular}{|c|c|c|c|c|c|c|}
\hline & \multicolumn{2}{|c|}{$r \gg 0$} & \multicolumn{2}{c|}{$r \ll 0$} & \\
State & $\wedge^{\bullet} \mathcal{E}$ & $H^{\bullet}\left(\mathbb{P}^{1}\right)$ & $\wedge^{\bullet} \mathcal{F}$ & $H^{\bullet}\left(\mathbb{P}^{1}\right)$ & Rep' & $\mathrm{U}(1)^{3}$ \\
\hline$\wedge^{2} W \otimes K_{(2,0)} U^{*}$ & 2 & 0 & - & - & $(\mathbf{3}, \mathbf{1}, \mathbf{1}, \mathbf{1})$ & $(0,0,0)$ \\
$\wedge^{2} V \otimes \wedge^{2} \tilde{V}^{*} \otimes K_{(1,-1)} U^{*}$ & 4 & 1 & - & - & $(\mathbf{3}, \mathbf{1}, \mathbf{1}, \mathbf{1})$ & $(0,0,0)$ \\
$\wedge^{2} V \otimes K_{(2,0)} \tilde{V}^{*}$ & - & - & 2 & 0 & $(\mathbf{1}, \mathbf{1}, \mathbf{1}, \mathbf{3})$ & $(0,0,0)$ \\
$\wedge^{2} U^{*} \otimes \wedge^{2} W \otimes K_{(1,-1)} \tilde{V}^{*}$ & - & - & 4 & 1 & $(\mathbf{1}, \mathbf{1}, \mathbf{1}, \mathbf{3})$ & $(0,0,0)$ \\
\hline
\end{tabular}

Table 17. List of all states which are not shared between the two phases.

states were all in non-integrable representations, and appear in pairs which cancel out of refined elliptic genera, suggesting that they are lifted in RG flow. In this fashion, we were able to confirm triality. (In principle, it could also happen that pairs of massive states become massless, but we did not observe this in any of the computed examples.) Finally, we were also able to support predictions for enhanced IR symmetries in certain theories, such as the enhanced $E_{6}$ in the $T_{2,2,2}$ theory, by confirming that the matching UV states fall into the decomposition of integrable representations of the $E_{6}$ algebra. 


\section{Acknowledgments}

We would like to thank A. Căldăraru, R. Donagi, A. Gadde, H. Jockers, S. Katz, A. Knutson, W. Lerche, V. Lu, I. Melnikov, and T. Pantev for useful discussions. Bei Jia was partially supported by NSF grant PHY-1316033. Eric Sharpe was partially supported by NSF grants PHY-1068725, PHY-1417410.

\section{A Bott-Borel-Weil}

As the Bott-Borel-Weil theorem plays a crucial role in our results, but is perhaps not well known in the physics community, this appendix will provide a short self-contained introduction.

First, we will use the notation $K_{\lambda} A$ for a vector space or bundle $A$ to denote a tensor product of copies of $A$ determined by a $\mathrm{U}(n)$ representation $\lambda$. For example, for the special case of $\mathrm{SU}(n)$ representations, we can associate representations with Young diagrams, and

$$
K_{\square} A=\operatorname{Sym}^{2} A, \quad K_{\square} A=\wedge^{2} A .
$$

More properly, a $\mathrm{U}(n)$ representation is determined by a non-increasing sequence of integers, as described in e.g. [9]. In the special case of $\mathrm{SU}(n)$ representations, those integers count the number of boxes in rows of Young diagrams. For example, for U(2),

$$
K_{(2,0)} A=\operatorname{Sym}^{2} A, \quad K_{(1,1)} A=\wedge^{2} A, \quad K_{(1,-1)} A=\left(\wedge^{\mathrm{top}} A\right)^{-1} \otimes \operatorname{Sym}^{2} A .
$$

The Bott-Borel-Weil theorem says that the only nonvanishing cohomology of $K_{\beta} S^{*} \otimes$ $K_{\gamma} Q^{*}$ over $G(k, V)$ lives in $\ell(\alpha)$, where $\ell(\alpha)$ is the number of 'mutations' required to transform $\alpha=(\beta, \gamma)$ into a dominant weight $\tilde{\alpha}$ of $G L(V)$. Furthermore,

$$
H^{\ell(\alpha)}\left(K_{\beta} S^{*} \otimes K_{\gamma} Q^{*}\right)=K_{\tilde{\alpha}} V^{*}
$$

Mutations are defined as follows: For

$$
\alpha=\left(\alpha_{1}, \alpha_{2}, \cdots, \alpha_{n}\right)
$$

a mutation is $[59$, remark 4.1.5]

$$
\sigma_{i} \cdot \alpha=\left(\alpha_{1}, \cdots, \alpha_{i-1}, \alpha_{i+1}-1, \alpha_{i}+1, \alpha_{i+2}, \cdots, \alpha_{n}\right) .
$$

In principle, we count the number of mutations needed to turn $(\beta, \gamma)$ into a nonincreasing sequence. If at any point there exists a mutation that leaves the sequence invariant, then all the cohomology vanishes.

For a simple example, consider the cohomology of $S=\mathcal{O}(-1)$ on $\mathbb{P}^{1}=G(1,2)$. Here, $\beta=(-1), \gamma=(0)$. Thus, we need to mutate $(-1,0)$ to a nonincreasing sequence. However, this sequence is invariant under mutation, hence, all the cohomology vanishes:

$$
H^{\bullet}(\mathcal{O}(-1))=0
$$


which is a standard result. More generally, the cohomology of $\mathcal{O}(k)$ on $\mathbb{P}^{1}$ can be computed as the cohomology of $K_{(k)} S^{*}$. To compute this cohomology, we mutate $(n, 0)$ into a nonincreasing sequence. If $k \geq 0$, no mutations are required, all the cohomology lives in degree zero, and so

$$
H^{0}(\mathcal{O}(k))=K_{(k, 0)} V^{*}=\operatorname{Sym}^{k} V^{*}
$$

for $V$ a two-dimensional vector space. Note that

$$
\operatorname{dim} \operatorname{Sym}^{k} V^{*}=k+1,
$$

and so the methods above predict that

$$
h^{0}(\mathcal{O}(k))=k+1
$$

for $k \geq 0$, in agreement with standard results. The case $k=-1$ we have already discussed. For $k<-1$, we can try the mutation

$$
(k, 0) \rightsquigarrow(-1, k+1) .
$$

If $n<-1$, then this is a nonincreasing sequence, and so we have

$$
H^{1}(\mathcal{O}(k))=K_{(-1, k+1)} V^{*} .
$$

Open Access. This article is distributed under the terms of the Creative Commons Attribution License (CC-BY 4.0), which permits any use, distribution and reproduction in any medium, provided the original author(s) and source are credited.

\section{References}

[1] K. Hori and D. Tong, Aspects of non-abelian gauge dynamics in two-dimensional $N=(2,2)$ theories, JHEP 05 (2007) 079 [hep-th/0609032] [INSPIRE].

[2] K. Hori, Duality in two-dimensional $(2,2)$ supersymmetric non-abelian gauge theories, JHEP 10 (2013) 121 [arXiv:1104.2853] [INSPIRE].

[3] R. Donagi and E. Sharpe, GLSM's for partial flag manifolds, J. Geom. Phys. 58 (2008) 1662 [arXiv:0704.1761] [INSPIRE].

[4] A. Caldararu, J. Distler, S. Hellerman, T. Pantev and E. Sharpe, Non-birational twisted derived equivalences in abelian GLSMs, Commun. Math. Phys. 294 (2010) 605 [arXiv:0709.3855] [INSPIRE].

[5] K. Hori and J. Knapp, Linear $\sigma$-models with strongly coupled phases - One parameter models, JHEP 11 (2013) 070 [arXiv:1308.6265] [INSPIRE].

[6] J. Halverson, V. Kumar and D.R. Morrison, New methods for characterizing phases of $2 D$ supersymmetric gauge theories, JHEP 09 (2013) 143 [arXiv:1305.3278] [INSPIRE].

[7] E. Sharpe, A few Ricci-flat stacks as phases of exotic GLSM's, Phys. Lett. B 726 (2013) 390 [arXiv: 1306.5440] [InSPIRE].

[8] H. Jockers, V. Kumar, J.M. Lapan, D.R. Morrison and M. Romo, Nonabelian 2 D gauge theories for determinantal Calabi-Yau varieties, JHEP 11 (2012) 166 [arXiv:1205.3192] [INSPIRE]. 
[9] B. Jia, E. Sharpe and R. Wu, Notes on nonabelian $(0,2)$ theories and dualities, JHEP 08 (2014) 017 [arXiv: 1401.1511] [INSPIRE].

[10] N.M. Addington, E.P. Segal and E. Sharpe, D-brane probes, branched double covers and noncommutative resolutions, Adv. Theor. Math. Phys. 18 (2014) 1369 [arXiv:1211.2446] [INSPIRE].

[11] E. Sharpe, Predictions for Gromov-Witten invariants of noncommutative resolutions, $J$. Geom. Phys. 74 (2013) 256 [arXiv:1212.5322] [INSPIRE].

[12] F. Benini, R. Eager, K. Hori and Y. Tachikawa, Elliptic genera of two-dimensional $N=2$ gauge theories with rank-one gauge groups, Lett. Math. Phys. 104 (2014) 465 [arXiv: 1305.0533] [INSPIRE].

[13] F. Benini, R. Eager, K. Hori and Y. Tachikawa, Elliptic genera of $2 d \mathcal{N}=2$ gauge theories, Commun. Math. Phys. 333 (2015) 1241 [arXiv:1308.4896] [INSPIRE].

[14] A. Gadde and S. Gukov, 2D index and surface operators, JHEP 03 (2014) 080 [arXiv: 1305.0266] [INSPIRE].

[15] H. Jockers, V. Kumar, J.M. Lapan, D.R. Morrison and M. Romo, Two-sphere partition functions and Gromov-Witten invariants, Commun. Math. Phys. 325 (2014) 1139 [arXiv: 1208.6244$]$ [INSPIRE].

[16] F. Benini and S. Cremonesi, Partition functions of $\mathcal{N}=(2,2)$ gauge theories on $S^{2}$ and vortices, Commun. Math. Phys. 334 (2015) 1483 [arXiv:1206.2356] [INSPIRE].

[17] N. Doroud, J. Gomis, B. Le Floch and S. Lee, Exact results in D $=2$ supersymmetric gauge theories, JHEP 05 (2013) 093 [arXiv: 1206.2606] [INSPIRE].

[18] I.V. Melnikov and E. Sharpe, On marginal deformations of $(0,2)$ non-linear $\sigma$-models, Phys. Lett. B 705 (2011) 529 [arXiv:1110.1886] [INSPIRE].

[19] L.B. Anderson, J. Gray and E. Sharpe, Algebroids, heterotic moduli spaces and the Strominger system, JHEP 07 (2014) 037 [arXiv:1402.1532] [INSPIRE].

[20] X. de la Ossa and E.E. Svanes, Holomorphic bundles and the moduli space of $N=1$ supersymmetric heterotic compactifications, JHEP 10 (2014) 123 [arXiv:1402.1725] [INSPIRE].

[21] X. de la Ossa and E. Svanes, Connections, field redefinitions, and heterotic supergravity, arXiv: 1409.3347.

[22] E. Svanes, Moduli in general SU(3)-structure heterotic compactifications, arXiv:1411.6696.

[23] M. Garcia-Fernandez, R. Rubio and C. Tipler, Infinitesimal moduli for the Strominger system and generalized Killing spinors, arXiv:1503.07562.

[24] A. Gadde, S. Gukov and P. Putrov, (0,2) trialities, JHEP 03 (2014) 076 [arXiv: 1310.0818] [inSPIRE].

[25] A. Gadde, S. Gukov and P. Putrov, Exact solutions of $2 D$ supersymmetric gauge theories, arXiv: 1404.5314 [INSPIRE].

[26] R. Donagi, J. Guffin, S. Katz and E. Sharpe, A mathematical theory of quantum sheaf cohomology, Asian J. Math. 18 (2014) 387 [arXiv:1110.3751] [INSPIRE].

[27] R. Donagi, J. Guffin, S. Katz and E. Sharpe, Physical aspects of quantum sheaf cohomology for deformations of tangent bundles of toric varieties, Adv. Theor. Math. Phys. 17 (2013) 1255 [arXiv:1110.3752] [INSPIRE]. 
[28] S.H. Katz and E. Sharpe, Notes on certain $(0,2)$ correlation functions, Commun. Math. Phys. 262 (2006) 611 [hep-th/0406226] [INSPIRE].

[29] E. Sharpe, Notes on certain other (0,2) correlation functions, Adv. Theor. Math. Phys. 13 (2009) 33 [hep-th/0605005] [INSPIRE].

[30] J. McOrist and I.V. Melnikov, Half-twisted correlators from the Coulomb branch, JHEP 04 (2008) 071 [arXiv: 0712.3272] [InSPIRE].

[31] J. McOrist and I.V. Melnikov, Summing the instantons in half-twisted linear $\sigma$-models, JHEP 02 (2009) 026 [arXiv:0810.0012] [INSPIRE].

[32] J. Guffin and S. Katz, Deformed quantum cohomology and $(0,2)$ mirror symmetry, JHEP 08 (2010) 109 [arXiv:0710.2354] [INSPIRE].

[33] E. Sharpe, Notes on correlation functions in $(0,2)$ theories, in String geometry (Snowbird, 2004), K. Becker et al. eds., American Mathematical Society, Providence, U.S.A. (2004), hep-th/0502064 [INSPIRE].

[34] J. Guffin, Quantum sheaf cohomology, a precis, arXiv:1101.1305 [INSPIRE].

[35] J. McOrist, The revival of (0,2) linear $\sigma$-models, Int. J. Mod. Phys. A 26 (2011) 1 [arXiv: 1010.4667] [INSPIRE].

[36] A. Adams, J. Distler and M. Ernebjerg, Topological heterotic rings, Adv. Theor. Math. Phys. 10 (2006) 657 [hep-th/0506263] [INSPIRE].

[37] J. McOrist and I.V. Melnikov, Old issues and linear $\sigma$-models, Adv. Theor. Math. Phys. 16 (2012) 251 [arXiv: 1103.1322] [InSPIRE].

[38] W. Lerche, C. Vafa and N.P. Warner, Chiral rings in $N=2$ superconformal theories, Nucl. Phys. B 324 (1989) 427 [inSPIRE].

[39] M. Bershadsky, S. Cecotti, H. Ooguri and C. Vafa, Kodaira-Spencer theory of gravity and exact results for quantum string amplitudes, Commun. Math. Phys. 165 (1994) 311 [hep-th/9309140] [INSPIRE].

[40] L.B. Anderson, B. Jia, R. Manion, B. Ovrut and E. Sharpe, General aspects of heterotic string compactifications on stacks and gerbes, arXiv:1307.2269 [INSPIRE].

[41] M. Bertolini, I.V. Melnikov and M.R. Plesser, Hybrid conformal field theories, JHEP 05 (2014) 043 [arXiv: 1307.7063] [INSPIRE].

[42] J. Distler, D.S. Freed and G.W. Moore, Orientifold precis, arXiv:0906.0795 [InSPIRE].

[43] J. Distler, D.S. Freed and G.W. Moore, Spin structures and superstrings, arXiv:1007.4581 [INSPIRE].

[44] K. Hori, H. Kim and P. Yi, Witten index and wall crossing, JHEP 01 (2015) 124 [arXiv:1407.2567] [INSPIRE].

[45] H. Blaine Lawson and M.L. Michelsohn, Spin geometry, Princeton University Press, Princeton U.S.A. (1990).

[46] T. Pantev, private communication.

[47] J. Distler, private communication.

[48] J. Scherk and J. Schwarz, Spontaneous breaking of supersymmetry through dimensional reduction, Phys. Lett. B 80 (1979) 60 [InSPIRE]. 
[49] E. Witten, Mirror manifolds and topological field theory, in Mirror symmetry I, S.T. Yau ed., American Mathematical Society, Providence U.S.A. (1998), hep-th/9112056 [INSPIRE].

[50] J. Distler and B.R. Greene, Aspects of $(2,0)$ string compactifications, Nucl. Phys. B 304 (1988) 1 [INSPIRE].

[51] T. Kawai and K. Mohri, Geometry of (0,2) Landau-Ginzburg orbifolds, Nucl. Phys. B 425 (1994) 191 [hep-th/9402148] [INSPIRE].

[52] M. Ando and E. Sharpe, Elliptic genera of Landau-Ginzburg models over nontrivial spaces, Adv. Theor. Math. Phys. 16 (2012) 1087 [arXiv:0905.1285] [InSPIRE].

[53] A. Căldăraru, private communication.

[54] W. Lerche, private communication.

[55] B. Kostant, Lie algebra cohomology and the generalized Borel-Weil theorem, Ann. Math. 74 (1961) 329 .

[56] B. Kostant, A generalization of the Bott-Borel-Weil theorem and Euler number multiplets of representations, in Conférence Moshé Flato 1999, volume 1, G. Dito and D. Sternheimer eds., Kluwer Academic PUblishers, Dordrecht The Netherlands (2000).

[57] P. Di Francesco, P. Mathieu and D. Senechal, Conformal field theory, Springer, Germany (1997).

[58] R. Slansky, Group theory for unified model building, Phys. Rept. 79 (1981) 1 [InSPIRE].

[59] J. Weyman, Cohomology of vector bundles and syzygies, Cambridge University Press, Cambridge U.K. (2003). 